Review

\title{
Positron Emission Tomography (PET) in Oncology
}

\author{
Andrea Gallamini ${ }^{1, *}$, Colette Zwarthoed ${ }^{2}$ and Anna Borra ${ }^{3}$ \\ 1 Department of Research and Medical Innovation, Antoine Lacassagne Cancer Center, \\ Nice University, Nice Cedex 2-06189 Nice, France \\ 2 Department of Nuclear Medicine, Antoine Lacassagne Cancer Center, Nice University, \\ Nice Cedex 2-06189 Nice, France; E-Mail: maracaz@hotmail.fr \\ 3 Hematology Department S. Croce Hospital, Via M. Coppino 26, Cuneo 12100, Italy; \\ E-Mail: annaborra@hotmail.it
}

* Author to whom correspondence should be addressed: E-Mail: gallamini.a@ospedale.cuneo.it.

Received: 30 April 2014; in revised form: 25 July 2014 / Accepted: 7 August 2014 /

Published: 29 September 2014

\begin{abstract}
Since its introduction in the early nineties as a promising functional imaging technique in the management of neoplastic disorders, FDG-PET, and subsequently FDG-PET/CT, has become a cornerstone in several oncologic procedures such as tumor staging and restaging, treatment efficacy assessment during or after treatment end and radiotherapy planning. Moreover, the continuous technological progress of image generation and the introduction of sophisticated software to use PET scan as a biomarker paved the way to calculate new prognostic markers such as the metabolic tumor volume (MTV) and the total amount of tumor glycolysis (TLG). FDG-PET/CT proved more sensitive than contrast-enhanced CT scan in staging of several type of lymphoma or in detecting widespread tumor dissemination in several solid cancers, such as breast, lung, colon, ovary and head and neck carcinoma. As a consequence the stage of patients was upgraded, with a change of treatment in $10 \%-15 \%$ of them. One of the most evident advantages of FDG-PET was its ability to detect, very early during treatment, significant changes in glucose metabolism or even complete shutoff of the neoplastic cell metabolism as a surrogate of tumor chemosensitivity assessment. This could enable clinicians to detect much earlier the effectiveness of a given antineoplastic treatment, as compared to the traditional radiological detection of tumor shrinkage, which usually takes time and occurs much later.
\end{abstract}


Keywords: FDG-PET; prognosis; oncology

\section{FDG-PET/CT for Tumor Staging}

Tumor staging is essential for a modern treatment strategy in oncology. An accurate tumor burden assessment at baseline is required to decide the optimal therapeutic strategy: whether the neoplasm is resectable or, due to a disseminated disease, only palliative treatment could be offered. Second, tumor burden is per se a prognostic factor and therefore staging at baseline turns out as a very important prognostic tool. Finally, disease extension definition is essential both for an early (that is, between chemotherapy cycles, often called "interim scanning") and final tumor response assessment, in the first case during adjuvant chemotherapy to identify ineffective treatment, in the second for final tumor response assessment after an effective treatment. Increasing numbers of patients with newly diagnosed cancer receive primary systemic therapy (so-called neo-adjuvant chemotherapy) followed by surgery. Histopathology provides an accurate assessment of treatment efficacy on the basis of the extent of residual tumor and regressive changes within tumor tissue. However, a variable proportion ranging from $20 \%$ to $40 \%$ of cancer patients achieve a pathologic complete response, a fact that necessitates methods for monitoring therapeutic effectiveness early during therapy. Interim scanning has an interesting clinical value, generally due to earlier changes in metabolic activity compared to variations in tumor size. Moreover shrinkage usually does not occur immediately after treatment, even in case of chemo-sensitive neoplasms [1]. FDG-PET/CT provides essential information regarding a response to primary chemotherapy: in this setting, baseline FDG-PET/CT is used as reference pre-treatment assessment of tumor extension. Interim FDG-PET/CT is compared to baseline FDG-PET/CT by visual or semi-quantitative assessment by standardized uptake value (SUV) calculation.

The SUV is calculated in a region of interest (ROI), as of the ratio of the FDG concentration in this area to the injected dose normalized to patient's body weight [2,3]; it is a relative easy calculation, frequently used in PET reporting and generally accepted as a semi-quantitative index for tumor

glucose metabolism. The most widely used parameter is $\mathrm{SUV}_{\mathrm{Max}}$, defined as the maximal SUV value in the ROI, and reduction in $\operatorname{SUV}_{\text {Max }}\left(\Delta \mathrm{SUV}_{\mathrm{Max}}\right)$ has been considered the most reliable indicator of the metabolic activity shutdown. A number of factors are known affecting the SUV calculation such as scanner calibration, clock synchronization between machine and injection time, patient body weight, fasting blood glucose level, image acquisition time, image reconstruction algorithm, partial volume effect, ROI definition [4-6]. More recently, new methods have been proposed to assess the burden of metabolically active tumor: the so-called Metabolic Tumor Volume (MTV) and the Total Lesion Glycolysis (TLG). Both parameters have been proposed as reliable indicators of the viable tumor bulk [7]. TLG is the product of median SUV value in a ROI ( $\left.\mathrm{SUV}_{\text {Mean }}\right)$ and MTV; it combines the anatomical and functional information of FDG-PET/CT [8]. The whole neoplastic burden could be assesses by the sum of MTV or TLG of the primary tumor, nodal and distant metastases. To calculate both quantitative parameters a preliminary tumor mapping with a manual contouring of all the tumor lesions by nuclear medicine physicians has been proposed. However, this procedure proved cumbersome when several lesions coalesce in a single bulky tumor mass, and time-consuming for 
the analytic measurement of $\mathrm{SUV}_{\mathrm{Max}}$ in every tumor lesion in advanced stage disseminated metastatic disease. New methods based on an adaptive threshold for $\mathrm{SUV}_{\mathrm{Max}}$ calculation (depending on tumor volume and tumor to background ratio), and semi-automated methods for tumor volume assessment have been proposed [9].

\subsection{Lung Cancer}

Staging Non-Small Cell Lung Cancer (NSCLC) by FDG-PET/CT is probably one of the main daily-practice indications encountered in a nuclear medicine department. Indeed, several non-invasive imaging modalities are available for staging NSCLC, but FDG-PET/CT utility and advantages have been clearly demonstrated long since. The addition of FDG-PET/CT to the conventional staging assessment was reportedly shown to change the management in $20 \%-30 \%$ of patients with NSCLC, mostly by upstaging disease and, notably, by redefining unresectable a previously defined resectable disease by traditional radiological means [10,11]. Several recent studies [12], and in particular the randomized multicenter study of Maziak et al. [13], reported that tumor staging with FDG-PET/CT immediately before surgery revealed more patients with mediastinal and distant metastatic disease than conventional imaging; disease was correctly upstaged in 23 of 167 FDG-PET/CT and in 11 of 162 conventional staging imaging modalities. Likewise, Fischer et al. noticed that the use of FDG-PET/CT for preoperative staging of NSCLC reduced both the total number of thoracotomies and the number of futile thoracotomies but did not affect overall mortality [14].

\subsubsection{T Staging}

FDG-ET/CT provides information on tumor staging according to TNM criteria. The utility of FDG-PET/CT for determining $\mathrm{T}$ stage, and in particular T3 or T4 invasion, has not been definitely determined. The evaluation of tumor spread to the pleura by FDG-PET/CT is probably the main advantage compared to conventional imaging. Actually, pleural effusion is relatively frequent in patients with NSCLC, and may be malignant or benign, in particular in patients with post-obstructive pneumonia. The sensitivity and specificity of FDG-PET/CT in determining pleural invasion range from $70 \%$ to $95 \%$ and $64 \%$ to $94 \%$ respectively [15]. The limitations of FDG-PET/CT for T staging are due to the anatomical localization and size measurement difficulties, microscopic disease underestimation, or absence of FDG uptake in case of low-metabolism tumors (bronchoalveolar cell carcinoma, carcinoid tumors). Nevertheless, FDG-PET/CT turned out the most accurate tool for $\mathrm{T}$ staging assessment, with a correct $\mathrm{T}$ staging definition in $86 \%$ of patients, as compared to $68 \%$ with computed tomography (CT) alone [16].

\subsubsection{N Staging}

Functional imaging with FDG-PET/CT proved to be superior to contrast-enhanced CT (CeCT) for $\mathrm{N}$ staging, in particular by adding metabolic information able to disclose morphologically undetectable nodal dissemination, ultimately increasing specificity and positive predictive value of $\mathrm{N}$ staging [17]. For example, in a prospective study (106 patients with NSCLC), the sensitivity, specificity and accuracy was higher with FDG-PET/CT (respectively 85\%, 84\% and 84\%) than with CeCT alone 
(respectively $70 \%, 69 \%$ and 69\%) [18]. However, the sensitivity of $\mathrm{N}$ staging by FDG-PET/CT remains disappointingly low (45\%) and false negative cases have been reported [19], particularly for lymph nodes size $<10 \mathrm{~mm}(\mathrm{Se}=32.4 \%)$ compared with lymph nodes $>10 \mathrm{~mm}(\mathrm{Se}=85.3 \%)$. Other limitations are the false-positive rates due to unspecific FDG uptake like inflammation or granulomatous disease (e.g., sarcoidosis), leading to a reduction in specificity [15]. Despite the above improvement in accuracy of $\mathrm{N}$ staging with FDG-PET/CT, surgical staging remains the standard, especially to detect occult mediastinal nodal invasion [20,21]. For these reasons, both endobronchial ultrasound-guided transbronchial needle aspiration (EBUS-TBNA) and endoscopic ultrasound-guided fine-needle aspiration (EUS-FNA) have been recommended as essential tools for tumor staging after FDG-PET/CT [22].

\subsubsection{Staging}

Long since, FDG-PET/CT proved very informative on metastatic spread in NSCLC, able to detect unsuspected distant metastases in up to $28 \%$ of patients [23], and to impact in a relevant way the treatment plan in as many as 53\% of cases [24]. FDG-PET/CT is for example useful for differentiating benign from malignant adrenal lesions, with a sensitivity and a specificity reported by Erasmus et al. of $100 \%$ and $80 \%-100 \%$ respectively [25], though in some cases a second imaging technique was needed [26]. FDG-PET/CT is also accurate for detecting bone metastasis, with an even higher accuracy than Magnetic Resonance Imaging (MRI) and bone scintigraphy (BS) in some publications [27].

\subsection{Colorectal Cancer (CRC)}

FDG-PET/CT is not routinely used in the staging of colorectal cancer, but could be proposed for problem solving, or in the presence of CEA elevation or resectable metastases [28], as proposed in the National Comprehensive Cancer Network guidelines (NCCN version 4.2013 [29]). Actually, for the local T staging, MRI and endorectal ultrasound (ERUS) are recommended [30]; for the detection of colorectal metastases ( $\mathrm{N}$ and $\mathrm{M}$ staging), the most frequently used modalities are US, CT, MRI and FDG-PET/CT [31].

In spite of the well-known limits of these metrics, ERUS, CT and MRI use a purely dimensional criterion to detect a nodal involvement [30]. However both criteria, functional and dimensional are still needed for $\mathrm{N}$ staging: FDG-PET/CT may provide additional metabolic information, but has limits essentially due to its spatial resolution, giving a lack of sensitivity. For example, in a Japanese study including 88 patients, FDG-PET/CT improved accuracy of preoperative lymph node involvement detection compared to nodal diameter, with a sensitivity and specificity of $51 \%$ and $85 \%$ for local lymph nodes and $62 \%$ and $92 \%$ for distant lymph nodes [32].

FDG-PET/CT seems to have only a limited value for M staging in CRC: for example, a meta-analysis of prospective studies (3,391 patients) aimed at assessing the role of imaging to detect liver metastases [33], and showed that MRI imaging is the preferred choice modality in patients who have not previously undergone therapy, in particular in evaluating lesions less than $1 \mathrm{~cm}$ (sensitivity $80 \%-88 \%$ and specificity $93 \%-97 \%$ ). FDG-PET/CT can be used as the second-line modality (sensitivity $81 \%-94 \%$, higher than $\mathrm{CT}$ ), but data about this modality were too limited for comparisons with others. 
FDG-PET/CT is not used routinely to detect lung metastatic spread of CRC; it may be accurate, especially when nodules have a sufficient size $(>9 \mathrm{~mm})$, with a sensitivity and specificity reported by Bamba et al. of $57.1 \%$ and $99.1 \%$ [34].

In patients with potentially operable metastatic colorectal cancer, FDG-PET/CT has a valuable role by improving staging accuracy and characterizing indeterminate lesions; in the study of Briggs et al., it could have a major impact on subsequent management in $30 \%$ of patients, and a minor impact in $12 \%$ of patients. Following FDG-PET/CT, as many as $35 \%$ of patients were no longer considered for surgery [35]. Likewise, another study of 341 patients with potentially resectable liver and/or pulmonary CRC metastases observed that FDG-PET/CT upstaged disease in 33.1\% and down staged disease in $24.9 \%$ compared to conventional imaging. As a consequence, surgery was averted in $33.8 \%$ patients, and FDG-PET/CT showed an overall sensitivity of $87.1 \%$ and specificity of $88 \%$ in detecting metastatic disease [36], in line with other observations [37,38].

A systematic review and economic evaluation recently concluded that there is insufficient evidence to authorize the use FDG-PET/CT routinely in primary CRC, but that using this imaging modality as a complementary imaging technique is cost-effective in the pre-operative staging of recurrent CRC (pooled sensitivity of $91 \%$ and specificity of $91 \%$ ) and in case of suspected metastatic disease (pooled sensitivity of $91 \%$ and specificity of $76 \%$ ). Although FDG-PET/CT may change patient management in some cases, we have to keep in mind that, at this time, the data are still discordant and the quality of studies is generally poor [39].

\subsection{Esophageal Cancer}

The role of FDG-PET/CT in esophageal cancer staging is still unsettled. According to the highlights of the EORTC St. Gallen International Expert Consensus on the primary therapy of gastric, gastroesophageal and esophageal squamous cell cancer (SCC) [40], there was uncertainty about the role of FDG-PET/CT as part of routine preoperative staging. Its value may rely in the detection of otherwise undiscovered distant metastases which could contraindicate the surgical approach, and in facilitating treatment planning for radiotherapy, but it is unclear if FDG-PET/CT scans add to the accuracy of state-of-the-art high quality CT to detect locally invasive tumour bulk. For the adenocarcinoma of the gastroesophageal junction and the esophagus, the prevailing recommendation is to stage patients with gold standard techniques such as endoscopy, CT, EUS and FDG-PET/CT.

For the local tumor extent, endoscopy, EUS, CT scan and MRI are classically recommended [41], and FDG-PET/CT alone is not routinely recommended. Both adenocarcinomas and SCCs have high FDG avidity, but false positive uptakes may be caused by esophagitis or post-dilatation, and false negative results may be encountered in small tumors [42]. EUS remains the imaging modality of choice for T staging because of its superior resolution.

For $\mathrm{N}$ staging, integrated FDG-PET/CT may improve the positive predictive value (PPV) of regional lymph nodes staging when compared with $\mathrm{CeCT}$ (PPV $=93.8 \%$ for FDG-PET/CT, versus $62.5 \%-73.7 \%$ for CeCT) [43]. Vazquez Sequeiros suggested that EUS is the most accurate technique for preoperative local-regional staging of esophageal carcinoma, once the CT and/or the PET have excluded the presence of distant metastasis [44]. According to this review, overall accuracy for $\mathrm{N}$ staging was $69 \%$ for CT, $56 \%$ for FDG-PET/CT, and $81 \%$ for EUS. EUS was the most sensitive 
technique, whereas CT and FDG-PET/CT were more specific tests. A more recent meta-analysis confirmed the limited accuracy of FDG-PET/CT for $\mathrm{N}$ staging, with a pooled sensitivity and specificity of $62 \%$ and $96 \%$ [45]. Another recent work underlined that the variable FDG avidity of the primary esophageal tumor could affect the detectability of lymph nodal metastases in esophageal cancer with a low metabolic activity [46]. FDG-PET/CT has a higher accuracy than CeCT for M staging; a meta-analysis observed that FDG-PET/CT has a sensitivity of $71 \%$ and specificity of $93 \%$ in the detection of distant metastases, in comparison to $52 \%$ and $91 \%$ for CT respectively [47]. When tumor spread was assessed with FDG-PET/CT using quantitative parameters such as $\mathrm{SUV}_{\mathrm{Max}}$, significant variation in TNM staging was evident especially for nodal lesions [48]. In a recent prospective study of 139 consecutive patients (all stages), FDG-PET/CT changed the stage group in 56 of $139(40 \%)$ patients and changed management in 47 of 139 (34\%) patients. FDG-PET/CT has also prognostic stratification in the primary staging of esophageal cancer [49].

Finally, baseline FDG-PET/CT was shown in preliminary reports, to have an independent prognostic value on esophageal cancer treatment outcome when PET-derived quantitative parameters were used for prognostic patient stratification. The latter included $\mathrm{SUV}_{\mathrm{Max}}$ and survival outcomes [50], FDG-PET/CT N stage [51] or pretreatment MTV and overall survival [52].

\subsection{Gastric and Gastroesophagal Cancer}

According to the highlights of the EORTC St. Gallen International Expert Consensus, the panellists agreed that there is currently no indication for FDG-PET/CT scans in routine staging of gastric cancer [40]. For preoperative T staging, EUS remains the choice modality, while both CT and PET are most useful to evaluate distant metastases [53], with variability sensitivity ranging from $33 \%$ to $81 \%$ and $47 \%$ to $81 \%$ respectively, and specificity ranging from $82 \%$ to $96 \%$ and $89 \%$ to $91 \%$ respectively. However, notably, FDG avidity was shown to depend on tumor histologic subtype; FDG-PET/CT has a significant lower sensitivity for diffuse type histology (mucinous, signet ring) than for the intestinal (non-mucinous) tumors [54]. In a similar way to some lymphoma subset, this observation may limit the value of FDG-PET/CT for staging in those cases. Another limitation of FDG-PET/CT staging is the false negative cases due to occult peritoneal dissemination. The rate of occult peritoneal disease varies from $20 \%$ to $25 \%$ in the literature [55], and laparoscopy and diagnostic washings prior to surgery may be warranted for patients who demonstrate advanced pathology at diagnosis (T3 or more, $\mathrm{N}+$ ) without evidence of metastatic dissemination.

\subsection{Pancreatic Cancer}

Multimodality imaging is critical in the diagnosis and management of pancreatic cancer (PC). FDG-PET/CT is increasingly viewed as a useful and accurate modality in diagnosing, staging and managing this neoplasm, but further studies are warranted at this time to confirm that [56].

For diagnosing PC, FDG-PET/CT has an acceptable pooled sensitivity and specificity of $91 \%$ and $81 \%$ respectively, according to a recent meta-analysis (30 studies, 1582 patients) [57]. The $\mathrm{SUV}_{\mathrm{Max}}$ of FDG-PET/CT can be used in the differential diagnosis of solitary pancreatic lesions and can also help in the prediction of proliferative activity of pancreatic cancer [58]. Indeed, in several observations, higher $\mathrm{SUV}_{\mathrm{Max}}$ of primary pancreatic tumor is associated with poor prognosis [59,60]; metabolic 
tumour burden like MTV and TLG may be some prognosis factors too [61]. As regards histologic subtypes, a recent study suggests that not only pancreatic ductal adenocarcinoma, but also solid pseudopapillary tumor has an increased FDG metabolism [62]. Like in other tumors, PC should sometimes be distinguished from inflammatory lesions mimicking cancer such as mass-forming pancreatitis: in this settings FDG-PET/CT proved of limited value either because the SUV Max values of the inflammatory lesion overlapped with those detected in pancreatic cancer [63], and the small volume of both lesions [64]. FDG-PET/MRI fusion image may significantly improve accuracy compared with that of FDG-PET/CT (96.6\% vs. 86.6\%) [65]. For evaluation of intraductal papillary mucinous neoplasms (IPMN), FDG-PET/CT seems to be promising in distinguishing benign from malignant lesions, and therefore for selecting patients for surgical treatment or for long-term follow-up [66,67]. For $\mathrm{N}$ and $\mathrm{T}$ staging of the disease, the accuracy of FDG-PET/CT remains unclear. According to the meta-analysis of Wang et al., the sensitivity and specificity for $\mathrm{N}$ stating are $64 \%$ and $81 \%$ respectively (four studies, 101 patients), and for liver metastasis 67\% and 96\% (seven studies, 316 patients) [57]. A number of reports focused on the role of FDG-PET/CT for early treatment response assessment, with interesting results for staging the disease [68]. For example, Topkan et al. observed that FDG-PET/CT alters initial management decisions in $36.6 \%$ of patients with locally advanced pancreatic carcinoma planned to undergo chemoradiation; in $26.8 \%$ of patients, FDG-PET/CT restaging showed distant metastases not detected by conventional staging [69].

\subsection{Head and Neck Squamous Cell Carcinoma}

The preoperative staging of squamous cell head and neck cancer (HNSCC) includes clinical examination and imaging methods like CeCT or MRI. Even these techniques could detect morphological invasion, small tumors are far better detected with FDG-PET/CT [70]. We should notice that necrotic lesions do not accumulate FDG, and diagnostic CeCT may be helpful for correct local staging in that case [71]. For $\mathrm{N}$ staging, a $\mathrm{N}$ plus stage cannot be ruled out by FDG-PET/CT, even with multiple time point dynamic imaging techniques, and surgeons should continue to rely on clinical evaluation to stage the patients and consider surgical staging if nodal metastases are clinically suspected [72]. Nevertheless, the clinical impact of FDG-PET/CT used for head and neck initial staging has been demonstrated in several publications. For example, in a prospective investigation (76 patients), FDG-PET/CT led to a TNM classification alteration in 34\%, and a change in radiotherapy planning technique and/or dose in 29\% [73]. Another observation related that the accuracy of PET and PET/CT for detecting primary tumors and cervical metastases was comparable, but significantly higher than that of CT/MRI (98\%-97\% vs. $86 \%-88 \%$ for primary; $92 \%-93 \%$ vs. $85 \%-86 \%$ for neck) [74]. Several other studies also demonstrate that pretreatment FDG-PET/CT is superior to conventional imaging and could alter the TNM stage in about 30\% [70]. Likewise, in patients with suspected recurrent head and neck cancer, a recent meta-analysis showed that FDG-PET/CT has high sensitivity (92\%), specificity (95\%) and accuracy (97\%) for screening distant metastasis before salvage treatment [75]. In addition to that, FDG-PET/CT may also detect other primary tumors like lung, gastric and esophageal cancer [76]. To summarize, FDG-PET/CT is a useful technique in HNSCC for planning the most appropriate treatment, offering the possibility to detect the primary tumor, locoregional and distant metastatic involvement, as well as another primary malignancy. 


\subsection{Breast Cancer}

The role of FDG-PET/CT in tumor staging and restaging is steadily increasing along with the body of evidence regarding its impact in breast cancer management [77]. The ability of FDG-PET/CT to detect primary tumor, locoregional and distant metastasis is described in current literature.

The initial breast cancer evaluation is commonly made by mammography, US and MRI. Even if multiple studies have shown the high accuracy of FDG-PET/CT in detecting suspected primary breast malignancy [78,79], we should remember that it is exclusively reserved as a staging tool for cases of pthology-proven disease [77]. Indeed, false negative cases have been reported, in case of tumor-size $<20 \mathrm{~mm}$, or lobular carcinoma subtype [79]. False positive cases could also be found, like fibroadenoma, inflammatory processes, gynecomastia, mastitis, granulomas, post-surgical changes, radiation necrosis or lactation [80]. Regarding the primary tumor, quantitative analysis has been correlated with histopathology characteristics. A higher $\mathrm{SUV}_{\mathrm{Max}}$ is correlated with more frequently detected distant metastases, aggressive histologic architecture, triple-negative receptor phenotype [81]. Groheux et al. also described the relationship between $\mathrm{SUV}_{\mathrm{Max}}$ and histologic grade (median of 9.7 for grade III) [82], and other studies noticed the more aggressive and prognostically poor invasive ductal carcinomas have increased average $\mathrm{SUV}_{\mathrm{Max}}$ relative to invasive lobular carcinomas [77].

For $\mathrm{N}$ staging, the imaging-guided sentinel lymph node biopsy (SLNB) with $\mathrm{Tc}^{99}$ colloid remains the gold standard, with high sensitivity and specificity for nodal disease detection. In patients with early-stage disease and clinically negative axillary nodes, the SLNB is recommended [83]. However, the search for a non-invasive technique has led to several studies evaluating the efficacy of conventional and/or functional imaging. The sensitivity of FDG-PET/CT in this application is variable in the literature. A meta-analysis (862 patients) observed a mean sensitivity of FDG-PET/CT of 56\% and a mean specificity of $96 \%$ [84]; PET performed less well for small metastases (mean sensitivity: $11 \%$ for micro metastases $\leq 2 \mathrm{~mm}$, and $57 \%$ for macro metastases $>2 \mathrm{~mm}$ ). In this study, MRI showed a higher sensitivity in detecting occult metastases than FDG-PET/CT. In another study (311 patients), a better sensitivity was observed (sensitivity $=82 \%$, specificity $=92 \%$ ) in evaluating axillary nodes [85]. Although the limited sensitivity of FDG-PET/CT for nodal staging, it generally outperforms conventional imaging regarding the detection of extra-axillary lymph node metastases [86]. Moreover, the specificity and positive predictive value of FDG-PET/CT are high (often exceeding 90\%) [77], which means that, in case of an FDG-avid axillary node, the sentinel lymph node procedure could be skipped and an immediate axillary lymph node dissection can be planned [87]. FDG-PET/CT may also detect other nodal metastases sites, like internal mammary chain and periclavicular chain [85]. It could therefore play a role in nodal staging of patients with clinical and/or histological risk factors. In pretreatment evaluation of breast cancer, several studies observed that FDG-PET/CT may change the initial staging compared to conventional imaging. For example, in a prospective study (106 patients with primary tumors larger than $3 \mathrm{~cm}$ ), FDG-PET/CT changed the initial staging in $42 \%$ of patients, and in $14 \%$ of patients, sites of extra-axillary malignancy were only detected by FDG-PET/CT. A treatment modification based on FDG-PET/CT results was done in 8\% [79]. Other publications underlined that FDG-PET/CT findings could have a clinical impact in breast cancer, like Riegger et al., who found that $14 \%$ of patients had a change in the disease management according to PET results [88]. In M staging, bone scintigraphy (BS) has traditionally been used as the first-line imaging technique for 
bone involvement despite its limited sensitivity for detecting pure lytic metastases [89]. Many studies suggest the superiority of FDG-PET/CT versus conventional BS, and the superiority or equivalence of FDG-PET/CT versus MRI imaging [77]. For example, in a study examining 132 bone lesions, the sensitivity of FDG-PET/CT was higher than BS (respectively 96\% and 76\%) [90]. FDG-PET/CT proved very useful for lung metastases detection, with the limits of a very low sensitivity for nodules with a largest diameter $\leq 8 \mathrm{~mm}$ [77], for visceral metastases, like adrenal masses with the exception of benign FDG-avid solid masses [91], or for liver metastasis, albeit MRI proved superior (like in colorectal cancer) [92]. Despite its high accuracy in breast cancer staging, FDG-PET/CT failed to prove cost-effective in baseline staging in the asymptomatic patient where other imaging modalities proved superior and could not be replaced by PET [77]. However, the benefits of FDG-PET/CT in the initial evaluation of breast cancer should be considered, and the choice of the staging imaging strategy should be done taking into account the clinical/histological risk factors.

\subsection{Other Gynecological Malignancies}

\subsubsection{Ovarian Cancer}

There is mounting evidence that FDG-PET/CT has an increasing role in the management of ovarian cancer, with its main indication to detect tumor recurrence in presence of rising CA-125 serum values and negative conventional imaging studies [93]. The benefits of the use of FDG-PET/CT in these settings has been reported several times in the literature [94,95], with a sensitivity of more than $90 \%$ in detecting occult metastases. In the study of Zimny et al., FDG-PET/CT preceded the conventional diagnosis by a median of 6 months in patients judged clinically free of disease. Menzel et al. suggest that a PET indication is worthwhile at CA 125 levels of approximately $30 \mathrm{U} / \mathrm{mL}$ [96]. A more recent prospective multi-center, cohort study (90 patients) confirmed the impact of FDG-PET/CT in suspected recurrent ovarian cancer, which affected disease management decisions in $60 \%$ of the cases (in $49 \%$ with a high, in $11 \%$ with a medium clinical impact) with a much higher detection rate compared to conventional imaging [97].

For the characterization of asymptomatic adnexal findings, FDG-PET/CT has no place due to lack of sensitivity [98], and MRI remains the best imaging modality choice.

For the initial staging of ovarian cancer, FDG-PET/CT is not routinely used. Nevertheless, some publications noticed that it could be interesting in advanced epithelial ovarian cancer, in particular for the detection of supradiaphragmatic lymph node metastases like parasternal lymph nodes, with better accuracy than conventional CT (detection rate: 67\% vs. 33\%) [99]. However, increased mediastinal FDG uptake was not shown to play a significant prognostic role, while complete cytoreduction did [100]. For the initial preoperative staging of ovarian cancer, FDG-PET/CT may be superior compared to CT alone [101,102], but some publications also observed limits, as De Iaco et al., who reported a sensitivity and specificity of 78 and $68 \%$ respectively, with a high rate of false negative results in lesions $<5 \mathrm{~mm}$ such as found in presence of peritoneal carcinomatosis [103].

However, conflicting results have been reported on the sensitivity of FDG-PET/CT scan in detecting peritoneal carcinomatosis; Turlakow, Suzuki and Kim reported higher diagnostic accuracy of FDG-PET/CT than CeCT in this settings, with a sensitivity and specificity for FDG-PET/CT of 
$67 \%-92.2 \%$ and $90 \%-94 \%$ respectively, as compared to $22 \%-88.5 \%$ and $65 \%-77 \%$ respectively for CeCT [104-106]. The sensitivity of FDG-PET/CT proved also similar to that of conventional MRI, and even better for detecting small peritoneal lesions $(<2 \mathrm{~cm})$ in patients with recurrent ovarian cancer [107]. However, FDG-PET/CT has limits, in particular for the detection of small peritoneal implants $(<5 \mathrm{~mm})$ because of the limited PET resolution, and surgical staging remains the gold standard [108]. The good performances of FDG-PET/CT in detecting peritoneal carcinomatosis lead to interesting information for optimizing patient selection for cytoreductive surgery in recurrent ovarian cancer; recently, Ebina et al. observed that FDG-PET/CT led to a change in management plan in $58.4 \%$ in that case, with a total number of patients in whom cytoreductive surgery was selected as the treatment of choice increased from 12 to 35 according to FDG-PET-CT results [109]. In the preoperative management, FDG-PET/CT is also able to detect distant metastases (25/95 patients upstaged from FIGO stage III to stage IV by FDG-PET/CT in a recent study [110]. However, upward stage migration did not worsen the prognosis of stage III patients, and in advanced ovarian cancer, the only prognostic factor that retained a significant prognostic value is the quality of response to cytoreductive therapy. Another study proposed FDG-PET/CT criteria such as FDG-PET/CT stage IV, pleural exudates, and PET-positive large bowel mesentery implants, which were statistically significant in the prognosis univariate analysis to guide the administration of neo-adjuvant chemotherapy in advanced ovarian cancer, but, once again, incomplete tumor debulking was the only statistically significant independent prognostic variable using multivariate analysis $(p=0.0001)$ [111]. Other prognostic factors like MTV or TGL may be interesting, but more data are needed at this time to confirm that [112].

\subsubsection{Uterine Cervical Cancer}

FDG-PET/CT has an increasing role in the primary evaluation of uterine cervical carcinoma, in particular for evaluating lymph node status and distant metastases [113].

The positive diagnosis of cervical carcinoma is established by clinical examination and cervical biopsy. The local extent of the disease is usually assessed by colposcopy, while parametrical and soft tissue invasion is detected by MRI, which has a high soft-tissue contrast resolution.

Cervical carcinoma is usually highly FDG avid, and the primary tumor may be observed on FDG-PET/CT, but its value is limited in defining local extent compared to MRI. On the other hand, FDG-PET/CT can be used for the initial evaluation of lymph node involvement [114], in particular in advanced stage disease. Indeed, FDG-PET/CT proved more accurate than CT alone for N staging, depending on the tumor stage [115], with sensitivity and specificity values of $53 \%-73 \%$ and $90 \%-97 \%$ respectively in early stage [116,117], and of $75 \%$ and $95 \%$, respectively in advanced stage [118].

A major issue in $\mathrm{N}$ staging is the relative weak sensitivity of anatomical and functional imaging for tumor detection, especially in case of microscopic nodal invasion, with a number of false negative results with FDG-PET/CT as high as $22 \%$ in case of para-aortic and pelvic occult metastases [119]. On the other hand, standard-technique MRI imaging showed a lower sensitivity than FDG-PET/CT (30.3\% vs. 57.6\%) [120], while Diffusion Weighted MRI (DW-MRI) proved superior (83.3\% vs. $38.9 \%$ ) [121]. Consequently, surgical staging is not necessary in presence of a positive FDG-PET/CT, while it remains the gold-standard procedure in case of a negative scan. Stage migration during 
cervical cancer staging occurs mainly for occult distant nodal invasion: nodal involvement at the highest level in para-aortic, retrocrural, supraclavicular areas is a significant prognostic factor [122]. The pre-operative tumor metabolic activity assessment by $\mathrm{SUV}_{\mathrm{Max}}$ measurement seems to have a prognostic role in uterine cervical cancer [114-123].

Despite significant improvement in tumor detection, FDG-PET/CT failed to supersede surgical staging in baseline tumor extension assessment. Gouy et al. [124] assessed the role of extended-field radiation on para-aortic nodes combined with chemotherapy in PET-negative patients, showing that disease outcome was similar to that of patients without extra-pelvic nodal invasion. These data seem to warrant the utility of surgical staging in PET-negative patients, albeit tumor spread in para-aortic nodes is rare in presence of PET negative scan ( $8 \%$ vs. $18 \%$ in PET-positive patients) [125].

FDG-PET/CT proved superior to conventional radiological techniques for $\mathrm{M}$ staging, like for example in case of bone metastases [126], leading to a modification in radiotherapy dose and fields in $34 \%$ and of the overall therapeutic strategy in $23 \%$ of the patients [127].

\subsubsection{Melanoma}

Several studies witnessed over the last decade the utility of FDG-PET/CT in the management of patients with advanced melanoma $[128,129]$. For example, in a study of 38 patients with melanoma stage II or III, a sensitivity of $97 \%$ and a specificity of $56 \%$ were calculated respectively for FDG-PET/CT, compared with $62 \%$ and $22 \%$, respectively for other imaging modalities, resulting in stage migration in $34 \%$ of the cases [130], suggesting that FDG-PET/CT could replace the standard battery of imaging tests currently performed on high risk melanoma patients $[131,132]$.

\subsubsection{Lymphoma}

The above-mentioned considerations on physiopathology of tracer uptake and mechanism of image generation in PET scan are the underpinnings of the functional imaging concept. Moreover, they could explain the higher accuracy of FDG-PET/CT in baseline lymphoma staging compared with traditional anatomical imaging techniques, such as CT or MRI. Fused FDG-PET/CT is by far the more accurate imaging strategy compared with CeCT or PET alone [133,134]. By upstaging one of three or four patients with lymphoma, FDG-PET/CT could, in theory, significantly improve final outcomes [135]. Most of the existing evidence in the literature on the contribution of FDG-PET/CT in lymphoma staging comes from studies performed in Hodgkin lymphoma (HL). FDG-PET/CT proved to be a more sensitive and specific imaging technique than other conventional modalities, including Gallium and CT for determination of extent of nodal and extranodal disease [136]. Stage migration occurs in nearly $25 \%$ of patients, mostly to upstage disease, leading to a change in treatment strategy in nearly $10 \%-15 \%$ of them [137]. The role of CeCT performed simultaneously in the same diagnostic session with the PET scan (PET/CeCT) is still a subject of debate. Direct comparison of unenhanced, low-dose, FDG-PET/CT and PET/CeCT did not show statistically significant differences in the number of detected nodal and extranodal sites, but lymphoma was occasionally upstaged with the help of $\mathrm{CeCT}$ or additional clinically relevant findings were identified [138,139]. PET/CeCT may be useful in patients with abdominal and pelvic involvement for delineating lymph nodes from adjacent bowel loops and vasculature [140]. 
As far as the contribution of functional imaging in Diffuse Large B-Cell lymphoma (DLBCL) staging, the overall accuracy of CeCT and FDG-PET/CT in lymphoma staging was assessed in a headto-head comparison of both techniques by Elstrom et al. in a mixed series of $37 \mathrm{HL}$ and 38 DLBCL patients. FDG-PET/CT detected additional lesions in one third of the patients staged with CT, and in $33 \%$ an increased clinical stage was demonstrated. Lymphoma therapy changed based on FDG-PET/CT in two patients. In contrast, diagnostic CT detected few splenic lesions, without any stage migration [141].

In the PET era, the role of bone marrow trephine biopsy (BMB) for lymphoma staging has been questioned. In a recent retrospective study, the role of routine BMB was assessed in a cohort of $454 \mathrm{HL}$ patients staged with FDG-PET/CT: BMB upstaged only five patients from stage III to IV and no BMB allocated patients in another treatment or risk group [142]. Although BMB remains essential for the diagnostic workup, bone marrow involvement is a rare finding at disease onset in HL, thus in patients staged with FDG-PET/CT, BMB should no longer be a routine procedure.

As for HL disease, FDG-PET/CT is being routinely used in DLBCL staging for its ability to evaluate nodal and extranodal disease sites like skeletal, liver and lung involvement. Recently, in a retrospective review conducted on 130 DLBCL patients, Khan et al. found a higher overall accuracy of FDG-PET/CT in detecting bone and bone marrow (B/BM) invasion by lymphoma compared with BMB, with a sensitivity and specificity of $94 \%, 100 \%$, and $40 \%, 100 \%$, respectively. The negative and positive predicting value was $98 \%$ and $100 \%$, respectively. However, these data should be interpreted with caution. In fact, the authors claimed that the criterion for bone marrow involvement was either histologically-proven DLBCL presence in the marrow biopsy or PET-positive B/BM involvement with a focal, focal and diffuse, or diffuse-only pattern of FDG uptake, irrespective of iliac crest biopsy or the pattern diffuse or focal of FDG uptake [143]. These results were somehow criticized or attenuated by Avigdor et al. who pointed out that bone marrow involvement detected by FDG-PET/CT has a similar Progression-Free Survival (PFS) and Overall Survival (OS) to those individuals with stage IV disease without an involved marrow, whereas marrow involvement identified by BMB was associated with a worse outcome [144]. In conclusion, although BMB involvement is largely predicted by FDG-PET/CT results, BMB remains essential for the diagnostic workup in DLBCL, especially in cases of discordant lymphoma where the low grade component of BM invasion could hardly be detected by FDG-PET/CT. Prognostic value of FDG-PET/CT in baseline staging also has been reported in Follicular Lymphoma (FL, grade 3 more specifically). In a mixed clinical-imaging prognostic score containing (1) osteo-medullar uptake; (2) $\mathrm{SUV}_{\mathrm{Max}} \geq 15$; (3) extranodal involvement and (4) largest diameter of lesion $\geq 7 \mathrm{~cm}$, number of nodal sites affected on PET $\geq 6$, Le Dortz et al. found that a score value $\geq 2$, whatever the Follicular International Prognostic Index (FLIPI) score, was the only predictive factor in multivariate analysis on treatment outcome. Furthermore, FDG-PET/CT detected more lesion than CT especially for lymph node involvement $(+51 \%)$ and extranodal lesions $(+89 \%)$ resulting in patient upstage in only $11 \%$ of the cases [145]. In the Italian prospective study FOLL-05, upward stage migration upon PET scan occurred in up to $62 \%$ of limited-stage FL patients, who otherwise would have been treated with radiation therapy alone, resulting in a treatment change in a relevant portion of the patients [146]. For Mantle Cell Lymphoma (MCL), the combination of $\mathrm{SUV}_{\mathrm{Max}}$ and MCL-adapted international prognostic index (M-IPI) was able to stratify patients into three prognostic groups: low (29\%; no relapse/progression), intermediate (42\%; median Event-Free Survival (EFS): 37 months), and high risk (29\%, median EFS: 22 months) of treatment failure 
$(p=0.004)$ [147]. High FDG-uptake was related to the presence of blastoid and large cellular variants of MCL, which are known to have a more aggressive disease course compared with common MCL [148]. Conversely, the low sensitivity of FDG-PET/CT in detecting bone marrow and/or gastrointestinal involvement by lymphoma did not lead to significant upward stage shift. In conclusion, FDG-PET/CT is strongly recommended before treatment onset for patients in routine staging workup in most lymphoma subset with the exception of marginal extranodal lymphoma, cutaneous lymphoma, and small lymphocytic/Chronic Lymphocytic Leukemia (SLL/CLL) lymphoma [149] (See Table 1).

Table 1. FDG-PET/CT for solid tumor staging.

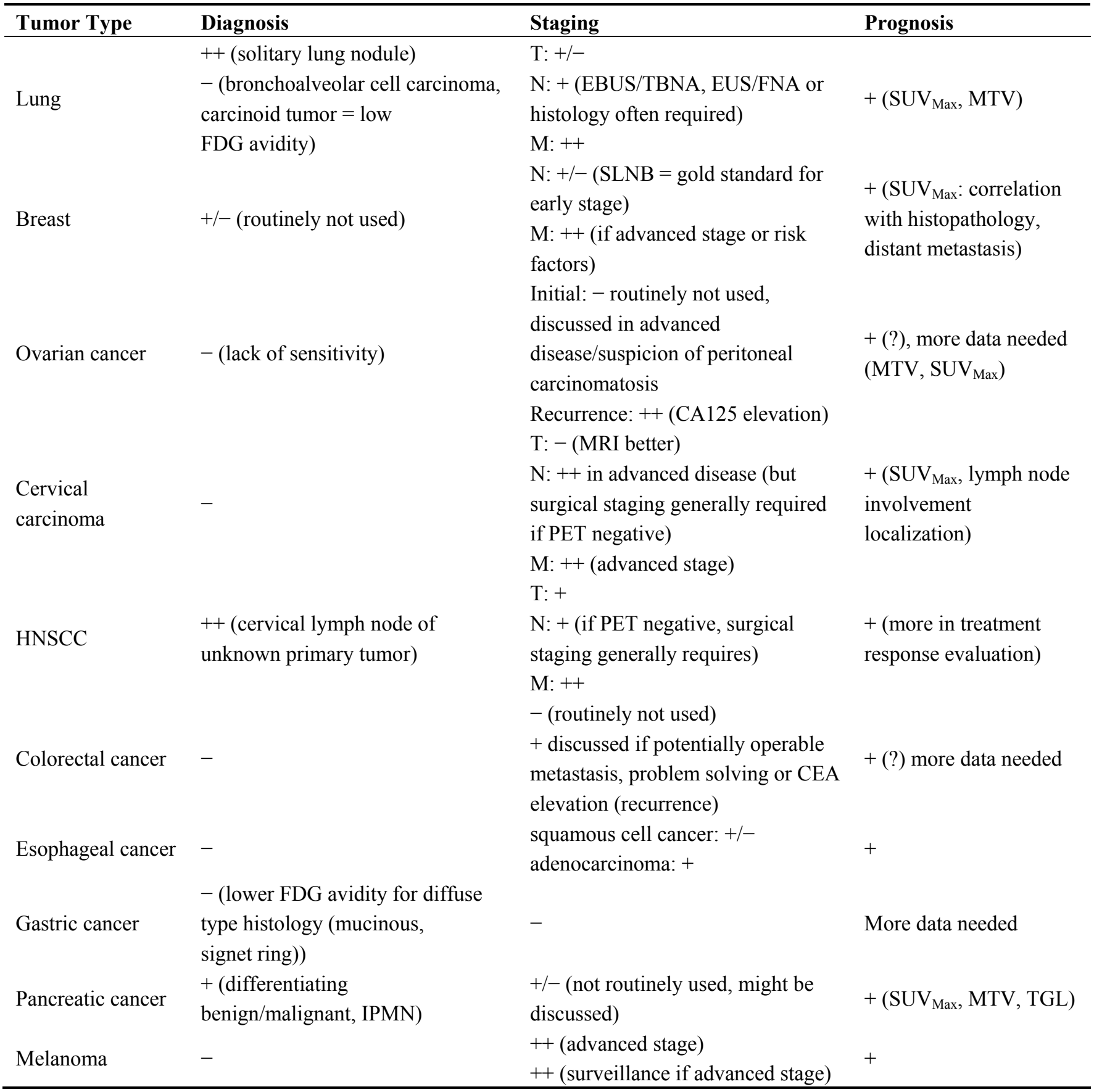

Legend of Table 1: -: PET not routinely recommended; +: for staging, PET indication may be discussed; interesting for prognosis; ++: PET recommended. 


\section{FDG-PET/CT in Solitary Pulmonary Nodule (SPN)}

Solitary Pulmonary Nodule (SPN) is one of the most frequent incidental findings $(14.8 \%$ of asymptomatic patients) [150], and could have both a benign or malignant origin. CeCT of high-resolution CT scan (HRCT) may give information on the morphologic characteristics of SPN (size, border, calcification, intra-nodular fat) and its size change, but this imaging modality has limits; $25 \%-39 \%$ of malignant nodules are inaccurately classified as benign [151,152]. The gold standard for diagnosing SPN is pathology, with a tissue sample obtained either surgically or by biopsy.

FDG-PET/CT is a non-invasive diagnostic tool which gives metabolic evaluation of the SPN, and may reduce the numbers of unnecessary samples.

A qualitative and quantitative (SUV measurement) assessment of the SPN metabolic activity can be interpreted on FDG-PET/CT. The $\mathrm{SUV}_{\mathrm{Max}}$ was shown to be predictor of the neoplastic nature of the tissue: in a large (585 patients) prospective study, 496 patients with a median $\mathrm{SUV}_{\mathrm{Max}}$ of 8.5 (range, 0 to 36) had a malignant neoplasm, and 89 patients with a median $\mathrm{SUV}_{\mathrm{Max}}$ of 4.9 (range, 0 to 28) had a benign lesion $(p<0.001)$. False negative FDG-PET/CT findings were: broncho-alveolar carcinoma (11), carcinoid (4), and renal cell (2). False positives findings were related to fungal infections (16) [153]. The threshold of $\mathrm{SUV}_{\mathrm{Max}}$ for distinguishing benign from malignant lesions is variable among the literature, and therefore lacks reproducibility. For example, Lowe et al. observed good performances of FDG-PET/CT with the most frequently $\mathrm{SUV}_{\mathrm{Max}}$ threshold used in the literature, which is 2.5 (overall sensitivity and specificity for detection of malignant nodules of 92\% and 90\%) [154].

A same cut-off value has been used in other publications, like Hashimoto et al., who calculated a sensitivity of $100 \%$, specificity of $63 \%$, positive and negative predictive values of $62 \%$ and $100 \%$, respectively. When an SUV of 1.59 was the cutoff for positive FDG-PET/CT results, the ROC analysis revealed a lower sensitivity (81\%), but higher specificity $(85 \%)$ [155]. In this study, the probability of malignancy in any visually evident lesion was about $60 \%$. Other studies suggest a higher cut-off value $\left(\mathrm{SUV}_{\mathrm{Max}}>3.5\right)$ [156], or a visual interpretation by experienced physician [157]. This variability among the different studies is probably explained by the fact that SUV may be affected by a large number of parameters, like equipment used, physic and biological factors. In most studies, the sensitivity of FDG-PET/CT is higher than its specificity [150]; FDG is a marker of glucose metabolism, and is not specific of neoplastic disease. Many benign abnormalities can produce false-positive findings on $\mathrm{PET} / \mathrm{CT}$, like granulomatosis, infection or inflammation. Consequently, in endemic regions of infectious or granulomatous lung diseases, FDG-PET/CT has significant limits. To give an example, a study of 279 patients in south-central United States with high prevalence of histoplasmosis, the specificity of PET/CT was only of $40 \%$ [158]. Another limit of FDG-PET/CT is its false-negatives rate encountered in case of small lesions $(<1 \mathrm{~cm}$, particularly $<7 \mathrm{~mm})$ [159], low tumor metabolic activity like bronchioloalveolar carcinoma [160], or hyperglycemia. Some authors have proposed dual-time point FDG-PET/CT imaging, using the change in SUV between early and delayed scans to help differentiate benign from malignant lesion. However, even if dual time point FDG-PET/CT appears to be more specific than single time point FDG-PET/CT ( $73 \%$ vs. 59\% respectively), the results of a recent meta-analysis indicate that dual time point FDG-PET/CT and single time point FDG-PET/CT have similar accuracy in the differential diagnosis of pulmonary nodules (Area Under Curve-AUC): 0.8244 vs. 0.8220) [161]. Despite of the limits of this imaging modality, FDG-PET/CT is one of the 
current mainstays of SPN evaluation. The ACCP evidence-based clinical practice guidelines (2nd edition) for the evaluation of patients with pulmonary nodules [162] recommend FDG-PET/CT if an indeterminate nodule is larger than $8 \mathrm{~mm}$ in a patient with low to moderate risk of malignancy (risk based on age, smoking history, exposure, cancer history). If CT scan shows growth of nodule, or patient has high risk of malignancy, tissue diagnosis is recommended.

\section{PET for Interim Tumor Response Assessment}

The definitive proof of the therapy effectiveness is improvement in survival. However, imaging is generally used to assess therapeutic effects earlier. Surrogate endpoints for survival should provide more objective and hopefully correct answers about the efficacy of treatment: the time to tumor progression and progression-free survival, when the disease recurs or progresses. Because cancers typically grow before they cause death, dimensional parameters provide readouts of tumor growth often considerably before the patients die of tumor itself. These metrics have been shown in some, but not all, cancers to be predictive of survival [163]. Current response assessment is based primarily on changes in tumor size measured with $\mathrm{CT}$ or other traditional radiological imaging modalities. The limits of anatomical imaging technique in tumor response evaluation will be mentioned in the next section of this article (see PET in tumor response).

The same limits of anatomical imaging are evident for tumor response monitoring during treatment: it appears intuitive that a reduction of tumor size after therapy indicates a better prognosis than does an unchanged or increasing tumor size. However, this assumption is not necessarily correct since the kinetics of tumor growth (and re-growth) after interim assessment could overtake the prognostic meaning of tumor size assessment in a given time point during treatment. Furthermore, tumor responses as assessed by radiological imaging modalities may be inaccurate because of errors in tumor measurements, errors in selection of measurable targets, and inter-observer variability of measurements [164]. For this reason, considerable efforts have been put in the last decade to redefine more reliable criteria. The progress in the knowledge of neoplastic cell metabolism prompted researchers the use of PET to assess the effect of cytostatic treatment on tumor cell metabolism objectively and quantitatively. First reports using planar FDG imaging for treatment monitoring were published more than $20 \mathrm{y}$ ago [165], and subsequent studies in the early 1990s suggested that tumor response might be identified earlier through changes in the FDG signal than through changes in measured size [166,167]. Because of a high tumor chemosensitivity, early tumor response assessment by functional imaging with a FDG-PET/CT scan performed after few cycles of chemotherapy has shown to predict with a very high accuracy the final treatment outcome in lymphoma [168-173]. Two main patterns of FDG uptake, resulting from different metabolic changes within the tumor, are observed in PET scans performed during chemotherapy in DLBCL and in HL. The first, resulting from a 'freezing' of the image fading during the chemotherapy-induced FDG uptake decline constitutes the net result of a dynamic balance between cell kill and re-growth; the second is a consequence of an abrupt and persistent switch-off of FDG uptake by the tumor and accessory cells [174]. These differences, depending on the number of chemotherapy cycles before interim PET and on a peculiar tumor histologic architecture, could also explain the variable performance of PET in these lymphoma subtypes [175-177]. 
A series of studies published in the millennium turnaround has stressed the higher accuracy of FDG-PET as compared to CT for monitoring tumor response to therapy because of its ability in differentiating residual viable neoplastic tissue from treatment-induced necrosis and fibrosis. Specifically, FDG-PET has been shown to be able to identify patients with a good response to therapy despite the presence of residual masses on CT. In malignant lymphoma as well as in several solid tumors, patients with negative PET results after completion of therapy have been found to have a favorable prognosis, in spite of evidence, in CT, of considerable residual tumor tissue.

In solid tumors, preoperative chemotherapy, the so-called neo-adjuvant treatment, has emerged in the last few years as a therapeutic approach to ease complete tumor resection and to improve final treatment outcome, with a consistent increase in disease-free survival. In this setting, patients without a response on FDG-PET may thereby undergo tumor resection earlier and avoid the side effects of ineffective therapy. Indeed interim PET proved as a reliable and robust prognostic tool for treatment adaptation in a number of solid neoplasms [178,179].

FDG-PET/CT has been extensively used for the evaluation of cytoreductive therapies. Several guidelines have been developed to permit quantitative or at least semi-quantitative assessments of changes, notably, the National Cancer Institute [180] and the EORTC (European Organization for the Research and Treatment of Cancer) guidelines [181]. It is expected that guidelines for patient preparation and protocols for image acquisition, reconstruction, and analysis will be broadly similar for treatment monitoring during cytoreductive therapies and cytostatic agents. For instance, it is just essential to perform a baseline scan before cytostatic treatment start, in order to pick up even a subtle FDG uptake change occurring during treatment. Some changes in FDG uptake could depend on pharmacodynamics, whereas others are associated with reduced tumor cell viability (depending on cytostatic treatment). For example, imatinib mesylate decreases tumor FDG uptake within hours to days of the commencement of treatment, whereas endocrine therapies, such as tamoxifen, increases FDG uptake within the same time frame. In general, effects occurring from hours to days after the initiation of treatment reflect pharmacodynamics (e.g., a direct effect on glucose transporter expression or hexokinase activity). Effects occurring after approximately 2-3 week or after 1-3 cycles of treatment are more characteristic of reduced cell viability [182].

Finally, before entering in the topic of interim tumor response assessment in different hematological and solid tumors, the reader should be informed on a number of warnings that should be used in interpreting the existing literature data. First, a consistent heterogeneity is found among different studies reporting on sensitivity and specificity of FDG-PET/CT for treatment monitoring. This depends mainly on the fact that some reports allude to sensitivity and specificity in detecting residual disease while other articles have focused on the ability of interim PET in detecting a tumor response [164]. Second, different metrics have been used to report PET results: Visual assessment of semi-quantitative methods. The former was used in chemosensitive disorders like lymphoma, where a complete response is observed in most cases after first-line chemotherapy [173,174].

In solid tumors, however, more subtle changes in the intensity of FDG uptake reduction occur, which are not easy recognized of standardized with visual assessment only [70]. Semi-quantitative methods comprehend tumor-to normal tissue ratio and SUV. Both parameters are affected by a number of variables, the most important being the image acquisition time. All these parameters have been the 
object of a wide debate, because SUV assessment and in general patients scanning across different PET centers lacks reproducibility and data comparison is impossible in this setting [183-185].

Early treatment response assessment with FDG-PET has been used as a helpful tool in a number of solid tumors (See Table 2).

Table 2. Indications of baseline and interim FDG-PET in principal solid tumor.

\begin{tabular}{ccc}
\hline Tumor & Initial Staging & Interim Assessment \\
\hline Breast & $+(+)$ & ++ \\
Lung (NSCLC) & $+(+)$ & ++ \\
Colorectal & -- & ++ \\
HNSCC & ++ & + \\
Esophagus & ++ & ++ \\
\hline
\end{tabular}

Legend of Table 2: ++: Generally useful; +: useful in selected cases; -: not useful.

\subsection{Lymphoma}

In HL, FDG-PET/CT enables an early evaluation of the metabolic changes occurring during the induction treatment as early as after the first [186], the second [170-172,187], and the third [188] cycle of chemotherapy (interim PET), with a predictive power on PFS even superseding the prognostic role of the International Prognostic Score (IPS) [189]. FDG-PET/CT yielded promising results as a surrogate of chemosensitivity for predicting tumor response also in DLBCL, albeit with a lower specificity [173,190-192], with an overall sensitivity and specificity of $43 \%-100 \%$ and $67 \%-100 \%$, respectively [174] for both lymphoma subtype. This high performance of interim PET is mainly due to the high chemosensitivity of lymphoma. At this writing, it is unknown whether a PET-adapted a strategy based on early chemotherapy intensification in patients with a positive interim PET scan could improve the final treatment outcome in the whole cohort of HL patients; several trials, designed to answer to this relevant, clinical question are underway [193]. As mentioned above, the predicting role on treatment outcome is higher in HL as compared to DLBCL, and differences in tumor pathobiology such as the neoplastic architecture and different ratio of neoplastic clone to microenvironment cells could explain this different performance [176]. Other reasons could be the wide range of the number of chemotherapy cycles received before interim PET, the difference in patient selection criteria (retrospective/prospective, first-line or second-line treatment), the different criteria for scan reporting, and whether interim PET was done only for observational aims or in the context of a PET-adaptive strategy. Finally, interim-PET was performed in other lymphoma subtype, such as FL [194] and in peripheral T-cell lymphoma [195] with interesting, but still preliminary results. In April 2009 at a workshop on interim PET in lymphoma held in Deauville (France), simple and reproducible rules have been proposed for interim FDG-PET/CT visual interpretation $[176,196]$, and these criteria have been recently retrospectively validated [197,198]. Briefly, the adopted rules include the following statements: (1) visual assessment is preferred, but SUV determination can be used in some cases; (2) interim PET interpretation should always be made by comparing the single foci of FDG uptake to those recorded in the baseline study; (3) the intensity of FDG uptake should be graded according to a five-point scale in which two reference organs, the mediastinal blood pool structures (MBPS) and liver, are used to define different grades of FDG uptake. Accordingly, the so-called five point scale has 
been proposed (Figure 1). The value of semi-quantitative FDG-PET/CT scan assessment was evaluated in the LYSA protocol LNH 2007-3B, in which interim FDG-PET/CT was performed after two (PET-2) and four (PET-4) cycles of chemotherapy and treatment adapted according to PET-4 results [173]. Optimal cutoff to predict treatment outcome was a $\triangle \mathrm{SUV}_{\mathrm{Max}}$ of PET-2 to PET-0 of $66 \%$ and of PET-4 to PET-0 of 70\%. Outcomes did not differ significantly if PET-2 and PET-4 were reported by visual assessment. On the contrary, a $\triangle \mathrm{SUV}_{\mathrm{Max}}$ value of PET-2 to PET- $0 \geq 66 \%$ identified patients with a significantly worse prognosis with a 2 -year PFS of $57 \%$ vs. $77 \%$.

\subsection{Breast Cancer}

Therapy response assessment in breast cancer patients is clinically relevant in cases with large and locally advanced tumors undergoing neo-adjuvant treatment with primary systemic chemotherapy. Schwarz-Dose et al. reported the $\mathrm{SUV}_{\mathrm{Max}}$ reduction $\left(\Delta \mathrm{SUV}_{\mathrm{Max}}\right)$ as semi-quantitative parameter to assess FDG-PET/CT predictive value with respect to pathological response [199]. A threshold of $45 \%$ reduction in SUV identified 11 of 15 responders, while the pathological non-responders were identified with a negative predictive value of $90 \%$ in the first cycle of chemotherapy. Martoni et al. and Keam et al. recently published similar results in patients undergoing neo-adjuvant chemotherapy [200,201]. Another unsettled issue is the optimal time point for interim FDG-PET/CT execution during neo-adjuvant chemotherapy treatment [1]. A number of studies reported a significantly higher $\mathrm{SUV}_{\mathrm{Max}}$ reduction as early as after the 1 st or 2 nd chemotherapy course in patients with histological evidence as compared to chemo-resistant ones [202-204]. Smith et al. in a small cohort of 22 breast cancer patients found that all the responders had a SUV reduction $\geq 55 \%$ as compared to pre-treatment value [203]. Overall accuracy in predicting treatment outcome after the 1 st and 2 nd cycle were $88 \%$ and $91 \%$, respectively.

\subsection{Colorectal Cancer}

Despite a curative intent, disease relapse often occurs in patients with stage III advanced colon cancer [205]. In this setting, chemotherapy seems to have no role, as disease could be eradicated by surgery or because of tumor chemo-resistance, in absence of predictors of treatment response.

Therefore a risk-adapted strategy with chemotherapy intensification for patients with a poor-prognosis disease while sparing toxicity for chemo-sensitive patients would be desirable and cost-effective.

In advanced colorectal cancer, a correlation between treatment outcome and FDG-PET/CT metabolic response after 1 or 2 months of chemotherapy has been reported [206-208], but results were considered inconsistent, mostly due to methodological issues in PET reporting in multi-metastatic disease [208]. Interim assessment as early as after one course of therapy seems to be the more promising approach with a very high negative predictive value (NPV) for non-responding patients a good and a high predicting value on overall survival [209]. However, procedure for scanner harmonization and protocol standardization for patient scanning image acquisition and reconstruction are in progress. For this reason a prospective multicenter clinical trial aimed at assessing the preoperative chemosensitivity testing with FDG-PET/CT as predictor of treatment benefit in Adjuvant stage III colon cancer has been launched on behalf of the Belgian Group for Digestive Oncology (BDGO), the so-called PePiTA Trial [205]. The trial will be conducted with a strict Quality Assurance and Quality Control program for scanner harmonization with a traditional ${ }^{18} \mathrm{~F}$ NEMA phantom and imaging generation and 
reconstruction to qualify PET centers to take part to the study. All the data will be centralized in the Core Lab of the study, at the Nuclear Medicine Department of the Jules Bordet Institute in Brussels.

\subsection{Head and Neck Squamous Cell Carcinoma}

The usefulness of interim FDG-PET/CT scanning in patients with HNSCC has been illustrated in a number of reports. Induction chemotherapy (ICT) has been used to select patients for organ preservation and determine subsequent treatments in patients with locally advanced HNSCC. Yoon et al. recently evaluated the efficacy of interim PET after ICT in HNSCC patients who achieved only partial response (PR) after ICT to predict clinical outcomes subsequent combined chemo-radiation [210]. A SUV Max $_{\text {of }} 4.8$ on interim FDG-PET/CT could predict final clinical CR after combined chemo-radiation $(100 \%$ vs. $20 \%, p=0.001)$, PFS (median, not reached vs. 8.5 months, $p<0.001$ ), and OS (median, not reached vs. 12.0 months, $p=0.001$ ) with a median follow-up of 20.3 months in surviving patients. The same held true for a $65 \%$ decrease in $\operatorname{SUV}_{\text {Max }}\left(\Delta \mathrm{SUV}_{\mathrm{Max}}\right)$ from baseline after ICT for final clinical CR $(100 \%$ vs. 33.3\%, $p=0.003)$, PFS (median, not reached $v s$. 8.9 months, $p<0.001$ ) and OS (median, not reached vs. 24.4 months, $p=0.001$ ) prediction. Ceulemans et al. prospectively compared the predictive ability of PET scan performed during radiotherapy to that performed 4 months after irradiation in a cohort of 40 HNSCC patients. The performance of interim was lower than end-of therapy FDG-PET/CT in terms of sensitivity (28.6\% vs. $78.6 \%, p<0.001)$, and negative predictive value (NPV) $(31 \% v s .60 \%, p<0.001)[211]$.

\subsection{Non-Small Cell Lung Cancer}

The number of patients presenting with NSCLC stage IV disease has increased over time [212]. This increase is most likely the result of better staging, because metastatic disease is identified long before it causes clinical symptoms [213]. Neo-adjuvant, preoperative chemotherapy for NSCLC has been extensively investigated, but its role in patient management remains controversial. Although responses to several courses of neo-adjuvant chemotherapy have been observed in up to $49 \%$ of the patients, no randomized trials have shown improvement in survival by preoperative chemotherapy and surgery as compared with surgery alone. FDG-PET/CT was reported to predict response to chemotherapy [214-216]. Hoekstra reported the results of a prospective multicenter study in which FDG-PET/CT was performed in 47 NSCLC patients before induction chemotherapy and after one and three cycles, and residual disease assessed in mediastinal lymph nodes [214]. Mediastinal lymph node status after induction chemotherapy by FDG-PET/CT predicted OS $(p=0.04)$ and PFS $(p=0.002)$. FDG-PET/CT was able to single out patients with different treatment outcome in the subset that showed treatment response according to CT. A positive FDG-PET/CT after one chemotherapy cycle predicted a poor PFS $(p=0.01)$. In a similar study, Decoster et al. prospectively assessed the predictive value of interim-FDG-PET/CT after 1 cycle of chemotherapy, compared to standard radiological assessment, according to the WHO recommendations. The concordance between the two imaging techniques was moderate (Spearman $r=0.62, p<0.01$ ). Surprisingly an early complete metabolic response did not improve patient overall survival [216].

More recently, several molecular-targeted agents such as the epidermal growth factor receptor tyrosine kinase inhibitors (EGFR-TKIs) erlotinib and gefitinib have emerged for treatment of NSCLC. 
Aukema et al. recently investigated the feasibility and the efficacy of early response monitoring with FDG-PET/CT during neo-adjuvant therapy with erlotinib in a small cohort of 23 NSCLC patients eligible for surgical resection [213]. Patients received preoperative erlotinib (150 mg) once daily for 3 weeks. FDG-PET/CT was performed before and at 1 week after erlotinib administration. Changes in tumor FDG uptake during treatment were prospectively assessed by $\mathrm{SUV}_{\text {Max }}$ measurement according to EORTC criteria [181]. According to these criteria, six patients $(26 \%)$ had a partial response within 1 week, 16 patients $(70 \%)$ had stable, and one patient $(4 \%)$ had progressive disease. The median percentage of necrosis in the resection specimens of treated patients was $40 \%$. In patients classified as "metabolic responders" ( $\left.\triangle \mathrm{SUV}_{\mathrm{Max}} \geq 25 \%\right)$, the median percentage necrosis in the metabolic responder group was $70 \%$, while the median percentage necrosis in metabolic non-responders was $40 \%$ $(p=0.09)$. In conclusion, early metabolic response corresponded to pathologic tumor regression in the resection specimen in most patients.

\subsection{Esophageal Cancer}

Esophageal cancer is among the ten most common malignancies worldwide and is associated with a high mortality [217]. In patients with locally advanced esophageal cancer, preoperative chemotherapy or chemo-radiotherapy has been shown to improve outcome with respect to survival. Patients who respond to neo-adjuvant therapy have a significantly improved survival, compared with patients who do not respond to the therapy; in this setting FDG-PET/CT could be the ideal tool to assess chemosensitivity early during treatment, but shared interpretation criteria are still matter of debate.

In one of the largest studies published so far on 211 consecutive patients who received neo-adjuvant chemotherapy followed by surgery, Miyata et al. reported the use of two semi-quantitative parameters for FDG-PET/CT scan interpretation: absolute post-treatment $\mathrm{SUV}_{\mathrm{Max}}$ value (post-SUV $\mathrm{Sax}_{\mathrm{Max}}$ ) and $\triangle \mathrm{SUV}_{\mathrm{Max}}$ of interim from baseline FDG-PET/CT [218]. FDG-PET/CT was performed before and 2-3 weeks after completion of neo-adjuvant chemotherapy. The mean reduction of $\mathrm{SUV}_{\mathrm{Max}}$ from baseline value ( $\triangle \mathrm{SUV}_{\mathrm{Max}}$ ) was 49.4 (from 11.4 to 5.8). $\Delta \mathrm{SUV}_{\mathrm{Max}}$ and the $\mathrm{SUV}_{\mathrm{Max}}$ value at the end of treatment (post-SUV $\mathrm{V}_{\mathrm{Max}}$ ) were able to predict pathologic response but not to distinguish partial from complete pathologic response. $\Delta \mathrm{SUV}_{\mathrm{Max}} \geq 50 \%$ was predictive of a superior 5-year overall survival $(56.5 \%$ vs. $39.6 \%, p=0.01)$ as did a post $\mathrm{SUV}_{\mathrm{Max}}<3.5(62.2 \%$ vs. $35.1 \%, p<0.0001)$.

Weber et al. monitored the response to treatment with FDG-PET/CT early in the course of therapy in 37 patients with locally advanced adenocarcinoma of the esophago-gastric junction [219]. FDG-PET/CT was carried out at baseline and 14 days after initiation of cisplatin-based polychemotherapy. For the quantitative assessment of therapy response, a circular ROI (diameter, $1.5 \mathrm{~cm}$ ) was placed over the tumor in the slice with the maximum SUV in the baseline scan. In the second FDG-PET/CT scan, the ROI was placed at the same position as in the baseline study. The authors showed that FDG-PET/CT allowed prediction of pathological response by metabolic response assessment as early as 2 weeks after the chemotherapy onset. They established a cutoff value of more than $35 \%$ in baseline mean SUV that allowed the prediction of clinical response with a sensitivity and specificity of $93 \%$ and $95 \%$, respectively.

On the basis of those findings FDG-PET/CT-response adapted clinical trials have been launched in single or multiple center settings. In the single-center MUNICON trial to prospectively evaluate 
the feasibility and potential effect on prognosis of administering FDG-PET/CT-response-guided chemotherapy 110 patients were evaluable for early metabolic response assessment after 2 weeks of induction chemotherapy [220].

Patients showing evidence of a metabolic response on FDG-PET/CT kept straight on with the original treatment for a maximum time of three months, while in case of no response patients underwent surgical resection two weeks later.

A histopathologic response was achieved in $58 \%$ of the metabolic responders. After a median follow-up of 2.3 years, median overall survival was not reached in metabolic responders, whereas median overall survival was 25.8 months in non-responders $(p=0.015)$. Early assessment with FDG-PET/CT of the response to therapy in patients with locally advanced adenocarcinomas has shown promising results in single-center studies and should now be evaluated in randomized, prospective multicenter trials. Such trials are very important because they (1) could pave the way to a possible implementation of this strategy in clinical practice; (2) establish the minimal requirement for inter-scanner calibration and standardization of image acquisition and reconstruction in order to make semi-quantitative parameters such as SUV reproducible and reliable [221].

\section{PET for Tumor Response}

\subsection{Radiological Imaging for Tumor Response}

The reduction in tumor size has been for decades the mainstay for monitoring the chemotherapy response in oncology. The World Health Organization (WHO) in the early eighties defined standard criteria for the assessment of tumor response [222]. The tumor bulk is defined as the sum of all nodal and extra-nodal lesions. The volume of nodal lesion should be measured by multiplying the 2 largest perpendicular diameters. The response to treatment is defined as a reduction $\geq 50 \%$ of the nodal and extranodal lesions. In case of complete disappearance of all the visible lesions the response is complete otherwise, the response is classified as a partial response. Twenty years later, the Response Evaluation Criteria in Solid Tumors (RECIST) for treatment response assessment [223], and subsequently, a revised version of the same criteria assessing a maximum of 5 tumor foci, vs. 10 in RECIST [224] was proposed, in order to simplify response assessment in clinical practice.

RECIST criteria were developed moving from the concept that tumor shrinkage is a surrogate for assessing tumor response. As in the WHO proposal, anatomical, cross-sectional imaging obtained with CT or MRI was the underpinning of this proposal, with a slight difference, stating that only the longest nodal diameter measurement was enough for assessing tumor bulk and chemotherapy response.

The issue of reproducibility of tumor size and tumor shrinkage measurement by using diameters of the lesions in trans-axial imaging techniques such as CT has been addressed by Moertel and Hanley in 1976 [225]. An experimental phantom was assembled filled with solid wood spheres embedded in a rubber matrix. Standard CT images were obtained and reproducibility of size measurement with rulers and calipers was checked among a panel of sixteen expert reviewers.

A disagreement on size measurement by $25 \%$ among reviewers on spheres with the identical diameter was present in $25 \%$ of reports and a disagreement of $50 \%$ in size in only $6.8 \%$ of cases. Although RECIST criteria have been used quite extensively in the past, some problems in tumor 
volume measurement were not the only pitfall in RECIST 1.0 and 1.1 versions: one issue is the choice of reducing intrinsically continuous phenomenon of tumor shrinkage and size reduction to a series of 4 levels of response (i.e., complete response, partial response, stable disease, and progressive disease). Having reduced a continuous phenomenon in few, pre-defined categories of response could have led to a loss of important information [226,227].

\subsection{The Problem of Residual Mass at the End of Treatment}

Another debated issue is the problem of residual mass at the end of treatment in several cancers and the inability of CT-based traditional radiological means to detect the persistence of tumor viable cells within a residual lesion harboring the tumor at baseline prompted the introduction of functional imaging for tumor response assessment in oncology.

This phenomenon was first described in lymphoma, where despite a good response to therapy, a residual mass can be demonstrated by radiological means in up to $80 \%$ of HL and in up to $40 \%$ of DLBCL patients after completion of treatment [228-230], even if only less then half of these masses will harbour residual disease [231]. Tumor masses often persist at the end of an adequate treatment for the presence of fibrotic tissue or extensive tumor necrosis. For the above reason the category of complete remission unconfirmed (RCU) was purposely proposed in the International Workshop criteria (IHP) for lymphoma response with the awareness that anatomic response criteria often underestimate the chemotherapeutic effect [232]. The significance of a residual mass at the end of treatment has been questioned also in a number of solid neoplasms. In HNSCC, Porceddu et al. [233] found the presence of a residual mass in 50/112 (45\%) consecutive patients in CR who achieved a complete response at the primary site. The patients underwent CeCT and FDG-PET/CT for nodal response assessment at 12 weeks after the end of (chemo) radiotherapy. Forty-one of 50 patients with a residual mass at CT showed a negative FDG-PET/CT at 12 weeks after therapy and none of them relapsed after a median follow-up of 28 months after CR entry. In patients affected by gastro-intestinal stromal tumors (GIST), a metabolic response of the tumor, documented by a negative FDG-PET/CT scan preceded by weeks the anatomical response detected on CT. By contrast, in presence of an active metabolic tissue, as observed in absence of FDG-PET/CT response at the end of treatment, is consistent with primary chemo-resistance. A positive FDG-PET/CT observed during follow-up in a patient with a completely negative FDG-PET/CT at the end of treatment points toward a secondary chemo-resistance [234].

Similar findings of very good or complete response to treatment in presence of a limited tumor size-response assessed by RECIST criteria, have been reported in hepatomas treated by sorafenib [235,236]. In the SHARP trial, the efficacy of sorafenib, and inhibitor of vascular endothelial growth factor (VEGF) and of platelet-derived growth factor (PDGF) was assessed in 602 patients affected by Hepatocellular Carcinoma (HCC) randomly assigned to assume sorafenib or placebo. Partial response by RECIST criteria was observed in $2 \%$ and $1 \%$, respectively. OS was slightly superior in the treated vs. placebo group (10.7 vs. 7.9 months, respectively; $p=0.001$ ); however, the median time to radiologic progression in the sorafenib was significantly higher than in the placebo group (5.5 vs. 2.8, respectively; $p<0.001$ ) [236]. In the same way in a number of hematological and non-hematological pediatric tumors the question whether a residual mass at the end of treatment is 
necessarily an harbinger of treatment failure could only be addressed only by functional imaging with FDG-PET/CT while traditional radiological imaging failed [237,238]. Thus, dimensional criteria proved to be no longer the more appropriate criteria in several neoplastic disorders.

The concept that tumor bulk decreases over time during cytostatic treatment was proven in experimental animal tumor models. Moreover, a strong relationship between FDG uptake entity and cancer cell number has been reported in a substantial number of studies $[239,240]$. Therefore, a decline in FDG uptake during tumor shrinkage results from reduction of viable tumor cell number, while a sustained increase in tumor activity with increasing SUV values is seen upon tumor regrowth. Such a relationship, however, is not linear and seems lost for very small tumor burden where the entity of the residual tracer uptake by the tumor is beyond the detection power of FDG-PET/CT scan. In these settings, the definition of complete response of the tumor to an ablative cytostatic treatment does not lie on objective findings but is inductive [241,242]. In most neoplastic disorders, the tumor mass at diagnosis corresponds to a weight of $10-100$ gr. or $10^{10}-10^{11}$ cells. Cytostatic drugs induce cell death by first-order kinetics in a constant fraction of the neoplastic population; a given dose will kill a fixed fractional number and not a constant absolute number of cells, whatever the dimension of the tumor. Thus a single dose of a cytostatic drug able to induce 1 log of neoplastic cell loss in a tumor bulk equivalent to $10^{11}$ cells should repeated 11 times to completely dissolve the tumor [243,244].

The resolution ability of the commercially available scanners is able to detect residual mass with a diameter ranging between 0.4 to $1 \mathrm{~cm}$, which translates in a tumor size corresponding to $0.1-0.5$ to 1 gr., or $10^{8}$ to $10^{9}$ cells [245,246]. As a consequence, the range detection of a FDG-PET/CT in tumor staging and restaging, for tumors in apparent complete remission, is only two logarithms. In conclusion, a complete metabolic response at the end of treatment could be observed in tumors with a very broad mass range, corresponding to a difference in neoplastic total cell number as large as 7 logarithms [163]. Thus, in spite of its favorable prognostic meaning a negative FDG-PET/CT could be compatible with the presence of residual tumor cells. More in general, FDG-PET/CT negativization at the end of treatment or, in some cases, even during treatment as observed in different types of cancer, should be considered not yet a complete tumor eradication but rather a very good prognostic indicator of treatment response and, possibly, long-term disease control achieved by an efficacious antineoplastic treatment.

Finally, three concepts should be well borne in mind: (1) several studies have shown that FDG-PET/CT is unsuitable to distinguish between minimal tumor burden vs. no tumor; (2) the scanners currently used are unable to detect microscopic residual tumor; (3) attempts to increase sensitivity of FDG-PET/CT by considering a residual FDG uptake an harbinger of residual tumor conflict with the presence of unspecific inflammation induced by chemo- or radiotherapy, thus compromising the specificity of this reading [163]. The same concepts could be extended to interim FDG-PET/CT during treatment: an negative interim FDG-PET/CT after few cycles of chemotherapy does not mean an early complete response (and disappearance) of the tumor at this time point, but rather an efficient fractional cell kill induced by the chemotherapy, leading, very likely, to a long-term tumor control by the chemotherapy itself. 


\subsection{Functional Imaging for Tumor Response}

The relationship between FDG uptake and tumor response was evaluated in pioneer studies where several scans performed during treatment documented progressive FDG uptake shutoff in responding cancers [166]. Thus a steadily decreasing FDG uptake over time, in most cases anticipates a complete pathological response at the end of treatment.

This tumor behavior, originally observed in breast cancer, is maintained across several other neoplasms such as lymphoma, lung cancer, HNSCC and esophageal cancers [163].

For all the above consideration, despite the important contribute of the traditional radiological means to document in a reproducible way the tumor bulk reduction induced by chemotherapy, the biologic predictive value of FDG-PET/CT appears to be greater in chemosensitive cancer such as lymphoma, lung cancer, mesothelioma, breast and esophageal cancer. For the above reasons, especially for the evident limitation of anatomical imaging for tumor response assessment, new criteria combining radiological and functional imaging have been proposed for treatment response assessment.

The EORTC criteria for tumor response assessment with FDG-PET/CT were proposed in the turn of millennium, in 1999. Briefly, a complete resolution of FDG accumulation is an indication of complete metabolic response. Partial metabolic response is characterized by more than $25 \%$ reduction of FDG uptake, stable metabolic disease shows an increase in FDG uptake of less than $25 \%$ or a decrease of less than $15 \%$, whereas an increase of more than 25 is attributed to progressive metabolic disease [181].

Ten years later, in an attempt to combine the anatomical and functional imaging results from RECIST and EORTC response criteria, new "mixed" criteria were proposed, the "PERCIST" —Positron Emission Tomography Response Criteria In Solid Tumors [163]. The most relevant innovation of these new criteria is that cancer response is expressed quantitatively as a continuous variable instead of being defined with four separate categories. The chosen unit of measure for this quantitative assessment is $\mathrm{SUV}_{\text {Peak }}$, the SUV value referred to a ROI of a sphere of tissue with a diameter of approximately $1.2 \mathrm{~cm}$ (to produce a $1-\mathrm{cm}^{3}$ volume spherical ROI). SUL (SUV corrected for lean body mass) is used instead SUV. Residual FDG uptake by the tumor is defined by the sum of SUL Peak in up to five tumor lesions. Residual pathological lesions should be compared with the corresponding baseline tumors and each baseline (pre-treatment) tumor SUL $L_{\text {Peak }}$ value must be $1.5 \times$ mean liver SUL +2 SDs of mean SUL. Due to a quantitative FDG-PET/CT assessment, FDG-PET/CT sites should adopt a protocol for patient scanning consistent with the National Cancer Institute and The Netherlands multicenter trial group recommendations, as well as a documented procedure for quality assurance and quality control for the procedures of scanner calibration [180,247].

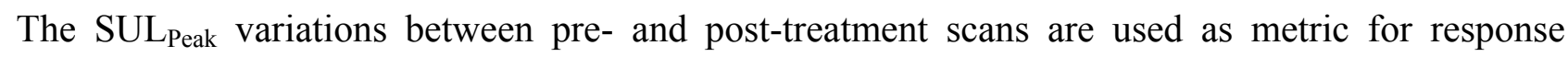
assessment. Metabolic complete response is defined as the complete clearance of all sites of FDG concentration. A partial decline $\geq$ than $30 \%$ of $\mathrm{SUV}_{\mathrm{Max}}$ and 0.8 unit decrease in SUL Peak between the most intense pre- and post-treatment lesions identify a partial response. The lesions considered are not necessarily anatomically coincident. An increase $\geq$ than $30 \% \mathrm{SUV}_{\mathrm{Max}}$ and a 0.8 unit increase in SUL $_{\text {Peak }}$ or the appearance of new lesions are the criteria for progressive disease. An adjunctive criterion for disease progression is an increase $\geq 75 \%$ of TLG. 
PERCIST criteria have been tested in limited reports in colorectal and in small cell lung cancer [248-250] and in bone metastases [251] with result similar or identical to EORTC classification. However, due to the complexity of the proposed quantitative assessment of residual tumor(s), and the logistic hurdles to be passed-by to put in place a thorough program of Quality assurance (QA) and quality control (QC) for scanner calibration in a multi-center settings, PERCIST has not been extensively used in clinical trial nor in the daily clinical practice.

In qualitative assessment of FDG-PET/CT scan performed at the end of treatment the intensity of FDG uptake in the residual sites still tracer-avid is compared to reference organs such as muscle, mediastinal blood pool, liver and brain. This interpretation key seems particularly attractive in neoplasms responsive to chemotherapy such as lymphoma, where a complete negativization of FDG-PET/CT scan is expected at the end of treatment in most cases. In this setting the IHP criteria have been proposed for end-of-treatment PET interpretation, combining the results of CeCT scan and FDG-PET/CT using both dimensional criteria with CT scan and dichotomized (positive or negative) FDG-PET/CT results [252,253]. These criteria, however, were validated only in a small cohort of 54 patients [254]. Moreover, dimensional criteria for lymph node size assessment were not fully reproducible, showing the least accuracy for diameters between 15 and $20 \mathrm{~mm}$ [255].

In 2009 during an international workshop of hemato-oncologists and nuclear medicine experts held in Deauville, simple and reproducible criteria for the interpretation of FDG-PET/CT performed interim during treatment by visual assessment were proposed for HL and DLBCL [196]. Here the residual FDG uptake assessed in the foci of persisting disease were compared to the FDG uptake in the so-called "reference" organs (mediastinal blood pool and liver), and scored along a five-point scale (5-PS) (See Figure $1[196,256])$.

Figure 1. Deauville score (DS).

\section{Deauville score (DS)}

- Score 1 no uptake

- Score 2 uptake $\leq$ mediastinum

- Score 3 uptake $>$ mediastinum but $\leq$ liver

- Score 4: moderately $\uparrow$ uptake $>$ liver

- Score 5 markedly $\uparrow$ uptake > liver and/or new sites of disease

Four years later, during the 12th International Congress on Malignant Lymphoma held in Lugano (Switzerland) in June 2013, a closed workshop attended by a group of haematologists and nuclear medicine experts was held with the aim of proposing and validating new response criteria for interim and end-of-treatment FDG-PET/CT in lymphoma, based on results of CeCT and FDG-PET/CT. Different criteria were proposed for FDG-avid and FDG-non avid lymphoma. For FDG-avid lymphoma ( $>90 \%$ of lymphoma subset) the 5-PS (See Figure 1) was proposed as interpretation key to 
quantify the entity of residual FDG uptake in FDG-PET/CT, along with dimensional criteria on CeCT. According to this proposal, patients with a score 1 to 3 were considered have complete metabolic response, patient with score 4-5 have partial response or progressive disease. Different from interim FDG-PET/CT, in which the 5-PS interpretation criteria were validated [197], in the case of FDG-PET/CT performed at the end of treatment these criteria were just a working proposal, awaiting confirmation in prospective studies. Furthermore, the choice to adopt the same cut-off value between score 3 and 4 to distinguish a negative from a positive FDG-PET/CT was somehow attenuated by the claim that "score 3 probably represents complete metabolic response (CMR), but patients may have different outcomes compared with score 1,2, depending on clinical context and treatment regimen" [257]. For non FDG-avid lymphoma (SLL/CLL lymphoma, extra-nodal marginal zone lymphoma, lymphoplasmacytic lymphoma, mycosis fungoides, and other cutaneous lymphoma), only classical dimensional criteria, like in RECIST, were proposed. In this lymphoma subset the size of the residual tumour bulk should be assessed on $\mathrm{CeCT}$, using the sum of the longest diameter of all the residual pathological lesions (See Table 3).

Table 3. New Lugano Criteria.

\begin{tabular}{|c|c|c|c|}
\hline $\begin{array}{c}\text { Response Assessment } \\
\text { at Interim }\end{array}$ & PET-CT Findings at Interim & $\begin{array}{l}\text { Remission Assessment } \\
\text { at End-of-Treatment }\end{array}$ & $\begin{array}{l}\text { PET-CT Findings } \\
\text { at End of Treatment }\end{array}$ \\
\hline $\begin{array}{l}\text { Complete Metabolic } \\
\text { Response (CMR) }\end{array}$ & $\begin{array}{l}\text { Score } 1,2 \\
\text { Score } 3 \text { also likely represents } \\
\text { a good response at interim but } \\
\text { an end-of-treatment scan is } \\
\text { recommended for further } \\
\text { evaluation. }\end{array}$ & $\begin{array}{l}\text { Complete Metabolic } \\
\text { Response } \\
\text { (CMR) }\end{array}$ & $\begin{array}{c}\text { Residual mass of any } \\
\text { size and Score } 1,2 . \\
\text { Score } 3 \text { should be interpreted } \\
\text { according to the clinical context } \\
\text { and pre-treatment prognosis but in } \\
\text { many patients indicates } \\
\text { a good prognosis/CMR with } \\
\text { standard treatment. For trials where } \\
\text { de-escalation strategies are being } \\
\text { investigated, it may be preferable to } \\
\text { consider score } 3 \text { as inadequate } \\
\text { response to avoid under-treatment. }\end{array}$ \\
\hline $\begin{array}{l}\text { Partial metabolic } \\
\text { response (PMR) }\end{array}$ & $\begin{array}{l}\text { Score } 4 \text { or } 5 \& \text { reduced } \\
\text { uptake from baseline }\end{array}$ & $\begin{array}{l}\text { Residual metabolic } \\
\text { disease (RMD) }\end{array}$ & $\begin{array}{l}\text { Score } 4 \text { or } 5 \text {, with reduced uptake } \\
\text { from baseline \& residual mass of } \\
\text { any size (but no new lesions) }\end{array}$ \\
\hline $\begin{array}{l}\text { No metabolic } \\
\text { response or } \\
\text { Progressive } \\
\text { Metabolic Disease } \\
\text { (NMR/PMD) } \\
\end{array}$ & $\begin{array}{c}\text { Score } 5 \& \text { no significant } \\
\text { decrease in uptake or } \\
\text { new FDG avid foci consistent } \\
\text { with lymphoma }\end{array}$ & $\begin{array}{l}\text { No Metabolic Response } \\
\text { or Progressive metabolic } \\
\text { disease (NMR/PMD) }\end{array}$ & $\begin{array}{c}\text { Score } 4 \text { or } 5 \& \text { no significant } \\
\text { change in uptake from baseline or } \\
\text { new FDG avid foci consistent } \\
\text { with lymphoma or increase in } \\
\text { uptake in previous disease foci }\end{array}$ \\
\hline
\end{tabular}

\subsection{Breast Cancer}

Two different clinical situations should be considered: the locally advanced, operable and the metastatic breast cancer. There is increasing clinical evidence for metastatic breast cancer and other advanced-stage solid tumors that FDG-PET/CT is the most accurate imaging procedure for 
assessment of the response at the end of treatment when both CT information and tumor metabolic activity are considered. In operable breast cancer chemotherapy treatment is administered before surgery. In this setting, the neo-adjuvant chemotherapy, (1) allows for a considerable tumor reduction before surgery; (2) can improve breast conservation rate [258]; (3) yields a pathological restaging in all the cases; (4) it achieves similar results to those obtained with initial surgery followed by conventional adjuvant chemotherapy in terms of disease-free survival, overall survival, and loco-regional control [259]. Although approximately $70 \%$ of operable locally advanced patients demonstrate a clinical response after neo-adjuvant chemotherapy, either on physical examination or on anatomic imaging, only 3\%-27\% achieve a complete histopathologic response [260-262].

Histopathology is often used as the reference standard for assessing the response to primary chemotherapy in breast cancer and pathologic complete response (pCR) is defined as the disappearance of the neoplastic cell in the biopsy tissue. However, Honkoop et al. found no difference in survival between patients with scattered microscopic foci of residual tumor cells and patients who achieved a pCR [263]. Moreover, despite the evidence of persisting neoplastic tissue, pathology seems to have a suboptimal predictive power: between $13 \%$ and $25 \%$ of patients in pCR experience a systemic recurrence within 5 years from pCR entry [264-266]. Furthermore, a number of patients showing persistence of neoplastic tissue do remain disease free for longtime. In metastatic breast cancer the most widely accepted and used surrogate parameter is tumor bulk reduction, usually assessed by RECIST criteria. However, since several cycles of treatment are needed before a change in tumor size can be assessed by anatomic imaging, functional imaging either at the end but also during treatment could, at least in theory, perform better [1]. A recent meta-analysis of the diagnostic performance of FDG-PET/CT in metastatic breast cancer included 18 articles published from January 1995 to June 2004: the median sensitivity was $92.7 \%$, and the median specificity was $81.6 \%$ [267]. Furthermore, comparisons with conventional imaging procedures used for staging (CT, US, and MRI) revealed that metabolic whole-body PET has a distinct advantage: the ability to assess tumor viability after previous treatments. A peculiar aspect is treatment efficacy evaluation of bone metastases because post-treatment healing is coupled with osteoblastic activity, ant the latter in turn, causes unspecific FGD uptake in the bone. Du et al. retrospectively analyzed the diagnostic contribute of FDG-PET/CT for bone metastases detection [268]. Briefly, 146 lesions were classified as osteolytic (77), osteoblastic (41), mixed (11) or negative (17) based on CT aspect. An increased FDG uptake ( $>80 \%$ of the cases in the first two abnormalities, $>60 \%$ in the third subset) was recorded on FDG-PET/CT. Most of them (80\%) as well as the CT-negative lesions showed a shutoff of FDG uptake upon treatment. Only 14 large lesions and nearly half (48\%) of the osteoblastic lesions maintained a sustained FDG uptake. Some of the formerly negative lesions became osteoblastic on CT. In conclusion, a clear discrepancy between morphologic aspect on CT and presence of viable neoplastic tissue on FDG-PET/CT was noted.

\subsection{Colorectal Cancer}

The role of imaging in colorectal cancer management has emerged in the last few years in parallel with the impressive progress in long-term disease control achieved by the modern treatment combining surgical resection and chemo-radiation. FDG-PET/CT has an important role in: (1) cancer (re)-staging in case of locally recurring or metastatic disease; (2) in detection of the site of recurrence in case of 
unexplained increase of CEA; and (3), in the assessment of residual masses after treatment. Here a only a brief mention will be made on treatment response monitoring after local ablative therapy of liver metastases, and monitoring radiotherapy and multimodality treatment responses in primary rectal cancer, while the emerging role of FDG-PET/CT in the prediction and evaluation of responses to treatment, such as monitoring chemotherapy responses has been reviewed in a detailed way in the section of cancer treatment monitoring.

Radical surgical resection is the preferred therapeutic option in advanced colorectal cancer with liver metastases. However, because of widespread metastatic disease dissemination in liver, complete resection with adequate tumor-free margins and adequate liver function reserve cannot be achieved in all patients. Selective loco-regional therapies have been proposed as alternative choice to radical surgery: cryosurgical ablation or cryotherapy (CSA) [269], radiofrequency ablation (RFA) [270], radioembolization using Yttrium ${ }^{90}\left({ }^{90} \mathrm{Y}\right)$ microspheres [271,272].

Different imaging techniques have been proposed for patient monitoring after loco-regional treatment of liver metastases [273-277]. FDG-PET/CT proved as a very accurate tool to discriminate unspecific inflammatory changes from persisting neoplastic uptake [273]. However problems arise in assessing treatment efficacy after radio-embolization with microspheres of ${ }^{90} \mathrm{Y}$. In this setting the preferred imaging technique is ${ }^{90} \mathrm{Y}$ bremsstrahlung CT-integrated single-photon emission computed tomography (SPECT) [278]. One drawback of this technique as, in general, for scintigraphy images is the low spatial resolution, for lesions $\leq 1$ centimeter [279-281]. Compared to SPECT/CT, FDG-PET/CT is a major step forward in metastatic liver disease detection after ${ }^{90} \mathrm{Y}$ microsphere treatment, allowing a direct imaging of ${ }^{90} \mathrm{Y}$ microspheres. Coincident ${ }^{18} \mathrm{~F}$ and ${ }^{90} \mathrm{Y}$ images could be acquired due to a decay of ${ }^{90} \mathrm{Y}$ to zirconium ${ }^{90}$, a $\beta+$ emitter [282-285].

The mainstay of therapy for colorectal cancer is radical surgery with radical resection of the mesial structures to prevent local and distant disease recurrence. However, despite the above radical surgical approach disease dissemination after surgery is still an ominous event. Locally advanced disease is the disease stage more frequently responsible for tumor spread and radiotherapy is often needed to improve disease control [286].

For locally advanced rectal cancer, neo-adjuvant chemoradiation has been proven successful [287,288]. Pathological examination of the resected tumor is the standard method to assess neo-adjuvant treatment outcome. Results are available only a few days after surgery and consequently cannot be used to guide individualized surgical procedure. However, first, accurate restaging to assess the success of pre-surgical neo-adjuvant treatment is mandatory for further treatment planning [289]. Two meta-analyses showed that CT, MRI and US are highly accurate in the staging of untreated tumors because of anatomic details to detect a peri-visceral or adjacent organ involvement [290,291]. The situation is completely opposed when these imaging modalities are intended to restage the tumor after chemoradiation, because very low accuracies ranging from only $30 \%$ to $60 \%$. Chemoradiation is expected to induce fibrosis or scarring of neoplastic tissue, and therefore radiological imaging in unsuitable for tumor restaging. On the other hand, tissue inflammation reduces the specificity of FDG-PET/CT since FDG selective uptake occurs not only in neoplastic but also in inflammatory tissue $[292,293]$. In a head-to-head study comparing anatomical with functional imaging, FDG-PET/CT predicted therapy outcomes significantly better than endorectal ultrasound, CT, and MRI. In the study of Amthauer et al. [294], FDG-PET/CT had a sensitivity of $100 \%$ and a specificity of $86 \%$ when 
a minimum post-therapeutic SUV reduction of $36 \%$ was used to define a response. The positive and negative predictive values were $93 \%$ and $100 \%$, respectively. Finally, Janssen et al. reported the results of a prospective study on FDG-PET/CT assessment of neo-adjuvant chemotherapy outcome in 51 patients with rectal cancer [295]: All patients underwent FDG-PET/CT imaging both before and 2 weeks after chemoradiation followed by local radical surgery. Tumor regression grade was calculated in resected samples according to Mandard criteria: grade 1-2 being considered pathological responders, grade 3-5 non-responders [296]. The best cutoff for value $\mathrm{SUV}_{\mathrm{Max}}$ reduction $\left(\Delta \mathrm{SUV}_{\mathrm{Max}}\right)$ in post treatment FDG-PET/CT scan to differentiate responders from non-responders was calculated by the ROC curve analysis. The most accurate cutoff value found in the training set for $\Delta \mathrm{SUV}_{\mathrm{Max}}$ was $48 \%$. The same value was found in the validation set, with an overall sensitivity to predict treatment outcome in the training and validation set of $64 \%-83 \%$ and $93 \%-100 \%$, respectively.

\subsection{Non-Small-Cell Lung Cancer}

Mortality for NSCLC has decreased dramatically in the last few years [297]. Since tumor stage remains in a multivariate analysis the only prognostic factor significantly correlated with survival among more than 169 different prognostic factors [298], the largest efforts to reduce mortality from this kind of tumor are directed to obtain an early tumor diagnosis: the earlier the diagnosis the better is the prognosis. Moreover, with the advent of functional imaging with PET the detection of unsuspected distant metastases became apparent much earlier and more patients once addressed to a curative treatment with a single modality became candidates to a more aggressive treatment because of a relatively good performance status. Moreover, fewer patients are likely to undergo futile thoracotomy for early stage disease and more patients are identified as requiring aggressive loco-regional or systemic treatment. At the same time the panoply of therapeutic options for NSCLC has dramatically widened, including more aggressive surgical techniques and the use of neo-adjuvant radiotherapy and chemotherapy regimens before surgery [299].

In a prospective study aimed at assessing the role of combined treatment with radiotherapy and chemotherapy in a cohort of 73 medically or surgically non-resectable, non operable, stage Ia to IIIb NSCLC, FDG-PET was compared with CeCT by MacManus et al. in treatment response assessment after radiotherapy or chemoradiotherapy [300]. Treatment response categories were defined as CR (complete response), PR (partial response), NR (non-response), PD (progressive disease). Concordant results were found in only $40 \%$ of the patients. Patients entering CR by CT were fewer than those in CR by FDG-PET/CT (10 vs. 34) and conversely more patients achieved PR or ND in the CT as compared to PET group (27 and two vs. 37 and 11, respectively. PD results were similar in both groups (10 vs. 9). Six cases were not assessable in the CT group. In multivariate analysis only PET response turned out significantly associated with a better survival as compared to classical prognostic markers $(p<0.0001)$. FDG-PET/CT showed a high predictive value on treatment outcome when erlotinib, an epidermal growth factor receptor (EGFR) inhibitor, was administered before radical surgery as neo-adjuvant treatment in NSCLC: in 70\% of the metabolic responders tumor necrosis had occurred, whereas necrosis was observed in only $40 \%$ of the non responders at 1 week after therapy [213]. Similar results were reported by Benz et al. [301] on FDG-PET/CT scan performed 2 weeks after the start of the neo-adjuvant erlotinib treatment, showing that an increasing metabolic activity in end of 
therapy PET was associated with a shorter time to progression (TTP) and a lower OS as compared to a longer TTP and OS for patients showing a stable of decreased FDG uptake (47 vs. 119 days and 87 vs. 828 days, respectively; $p<0.01$ and $<0.001$, respectively.

\subsection{Head and Neck Squamous Cell Carcinoma}

Several reports aimed at assessing the predictive value on treatment outcome FDG-PET/CT after radiation or combined modality treatment in HNSCC have been published. Prestwich et al. retrospectively assessed the overall accuracy of FDG-PET/CT in response assessment in locally advanced HNSCC for primary tumors or nodal disease [302]. The sensitivity, specificity, positive predictive value and negative predictive value for primary tumor and nodal disease were $100 \%, 89 \%$, $43 \%, 100 \%$, and $100 \%$, and $92 \%, 63 \%$, and $100 \%$, respectively. Due to this high performance the authors concluded the FDG-PET/CT performed in this setting could be decisional for HNSCC patients. In another study the role of FDG-PET/CT at the end of treatment of HNSCC was prospectively assessed: when FDG-PET/CT was performed 2 weeks after radiation therapy with a sensitivity and specificity of $86 \%$ and $85 \%$, respectively in a small cohort of 32 patients [303]. For relapse detection at 4 months, the sensitivity was reported to be $92 \%$ before treatment and 2 weeks and 4 months after treatment. Porceddu et al. assessed the value of FDG-PET/CT to predict the presence of residual viable tumor tissue within CT-detected residual masses three month after chemo-radiotherapy in a cohort of 112 consecutive HNSCC patients [233]. Fifty patients had a residual mass detected on CT; in 41 of them the FDG-PET/CT was negative. After a minimal follow-up of more than two years, none of these 41 with a FDG-negative residual mass and none of the 21 PET-negative patients without a residual mass experienced treatment failure.

\subsection{Epithelial Ovarian Cancer}

Ovarian carcinoma is the first cause of death in gynecologic neoplastic disorders characterized by a widespread presentation, an aggressive course and a very dismal prognosis, with less then $44 \%$ of the patients surviving more than 5 years after diagnosis. Nearly half of the patients acquire complete remission after first-line treatment and the median PFS is about one year and a half [304]. Treatment failure is observed in $20 \%-30 \%$ of the cases due to early disease progression or no treatment response. A similar proportion of patients experience disease relapse within the first 6 months after treatment end [305]. In ovarian carcinoma as well as in several other neoplasms a prompt diagnosis of disease recurrence is recommended in order to start as soon as possible a new salvage treatment. In this perspective, several combinations of imaging techniques with serial monitoring, a number of biomarkers have been proposed for a prompt diagnosis of disease relapse.

Significant controversy exists as to whether an earlier diagnosis of impending relapse with any of these modalities ultimately could improve patient survival [306-309]. FDG-PET/CT performs better than CeCT and MRI, particularly in the setting of suspected recurrence [310,311] and proved very useful in treatment decision to identify suitable patients for surgical resection from those in which chemotherapy is the only possible option [312,313]. In two small retrospective studies FDG-PET/CT was able to detect tumor relapse in presence of rising levels of CA 125 and equivocal CT findings or in case of symptoms and a negative CT, respectively [312,314]. In the first study FDG-PET/CT proved 
more accurate and precise than $\mathrm{CT}$ alone for localizing relapsing tumor with a sensitivity and specificity of $94.5 \%$ and $100 \%$, respectively [311]. Furthermore, in the study of Bhosale et al. $31 \%$ of patients with no evidence of disease on CT had lesions present on FDG-PET/CT [314].

However, subsequent studies showed the inability of FDG-PET/CT to detect small volume disease $<1 \mathrm{~cm}$ with FDG-PET/CT [315]; in those cases, CeCT scan should be planned whenever an increasing value of serum CA 125 is recorded or in presence of symptoms related to an impending relapse. FDG-PET/CT would be considered as a second-option imaging technique for patients candidates to second-line chemotherapy.

\section{MTV in Oncology}

Quantitative parameters for FDG-PET/CT interpretation proved as very robust prognostic parameters for treatment monitoring in oncology [163]. SUV is very easy to measure; it is readily available during PET interpretation and is an operator-independent variable. Its value has been considered to be strictly dependent from tissue glucose metabolism, provided that FDG-PET/CT are acquired in a standardized manner and proper scanner calibration procedures have been set up. Since the origin, different semi-quantitative and quantitative/kinetic analyses have been used to assess tumor metabolic response [316,317], but the methodology for determining total lesion glycolysis is still evolving; Different protocol for metabolic tumor volume assessment have been proposed with varied complexity of mathematic analysis, ranging from the more complex models of kinetic studies to methods based on tumor to background gradient of FDG uptake [316-318]. While full kinetic analysis required advanced sophisticated mathematical models and dynamic image acquisition, FDG-PET/CT allows a direct assessment of residual viable cell at a given time point during treatment. Moreover, a high tumor-to-background ratio encountered in most malignancies allows straightforward, automated and reproducible tumor delineation and determination of MTV. Interestingly, upon MTV multiplication

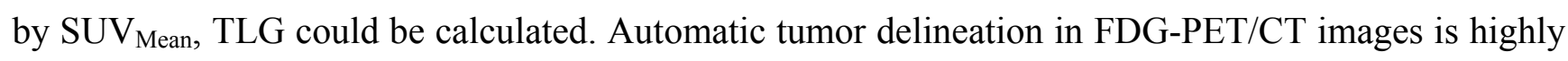
desirable for improved quantification, objective patient monitoring, and refinement of CT-based treatment planning in radiotherapy. However, the tumor segmentation task is challenging given the modest spatial resolution and the relatively high noise level in PET images. A large number of approaches have been proposed to segment tumors in PET images and the relative advantages or drawbacks discussed [7]. To date, there is no consensus on which method should be preferred for tumor segmentation, because of the difficulty in assessing tumor volumes in vivo [319]. Moreover, comparing the performance of these methods from the data published in the literature is almost impossible given the variety of situations in which evaluation studies have been conducted [7]. Finally, comparison of different segmentation methods on phantom-based studies could suffer from the impossibility of consider respiratory motion and heterogeneity of tumor FDG uptake.

\subsection{The Visual or Gradient Segmentation Method: The First One Applied and Still Widely Used}

The sharp variation of activity measured across the external edge of the tumor (the so-called tumor to background ratio: $\mathrm{T} / \mathrm{B}$ ) is the underpinning of the methods this gradient to calculate the tumor volume. Other similar, but more sophisticated methods including a denoising or deblurring filter or based on T/B gradient estimation have been proposed [320]. The tumor segmentation MIM (Software 
Inc., Cleveland, OH, USA) vista based on tumor to background gradient described by Werner-Wasik et al. relies on calculation of spatial derivatives measured along six radii moving from a starting point in the centrum of the tumor lesion, identified by the operator [321]. The measurement of $\mathrm{T} / \mathrm{B}$ gradient along these six radii provides the measures for tumor calculation. It has the advantage of being easily applicable from a technical point of view but is affected by a significant inter-observer variability [322] and it is time consuming.

\subsection{Fixed-Percentage Threshold Segmentation Method}

A totally different approach is used to measure the tumor volume based on a fixed threshold of $\mathrm{SUV}_{\mathrm{Max}}$ method. In brief, all the voxel measured within a pre defined sphere inside a given tumor mass exceeding a pre-defined percentage of $\mathrm{SUV}_{\mathrm{Max}}$ value are considered to measure the tumor volume of this mass. Cross sectional circles in three orthogonal plans are considered to cover all the mass volume [321]. Due to biological and physical factors there are no "normal" values for SUVs to be similarly used in every case. It has been shown that this method often fails, e.g., when the physiological background activity lies above the fixed threshold [323].

\subsection{Maximal Intensity Threshold}

New methods for tumor segmentation have been proposed such as the maximal intensity threshold, based on calculation of all the voxel exceeding an absolute $\mathrm{SUV}_{\mathrm{Max}}$ value. These methods were deemed more accurate for tumor identification and volume measurement compared to fixed-threshold ones $[323,324]$. However, criticism against these conclusions arose from the phantom studies, showing a superiority of gradient segmentation model for tumor quantification [321]. The error was low $(<5 \%)$ for tumor lesions with a diameter $>$ than $2 \mathrm{~cm}$. whatever the segmentation method used, while a significantly lower error value (8.2\%) was found adopting a $\mathrm{T} / \mathrm{B}$ gradient method compared to a $45 \%$ maximal intensity method for lesions with a diameter $\leq 2 \mathrm{~cm}$. Moreover, despite background noise measurement depends on acquisition time, the later does not seem to affect the results of $\mathrm{T} / \mathrm{B}$ gradient methods for MTV calculation [321].

\subsection{Adaptive Threshold Segmentation Method}

According to this method initial tumor volume estimate is determined on CT images. Approximate source to background (S/B) are then obtained for the corresponding tumor lesion on PET images. From the $\mathrm{CT}$ estimate of the lesion size and the PET estimate of the S/B ratio, the appropriate optimum threshold is chosen. The threshold is applied to PET images to obtain lesion activity and a final estimate of the lesion volume [325].

\subsection{Methods of Tumor Contour}

To calculate MTV and TLG a preliminary tumor mapping with a manual contouring of all the tumor lesions by nuclear medicine physicians has been proposed. However, this procedure proved cumbersome when several lesions coalesce in a single bulky tumor mass, and time-consuming for the analytic measurement of SUV $\mathrm{Sax}_{\text {in }}$ every tumor lesion in advanced, stage disseminated metastatic 
disease. With the semi-automatic method only two manual interventions are needed: the first consists in the identification of spatial orthogonal axes of the tumor mass. Then the dedicated software automatically calculated the tumor volume along these three axes as in MIM vista method (see above), and finally the operator manually adjusts the shape of the contour to fit the anatomical edge of the tumor mass [9].

\subsection{Other Methods}

Many new methods are still threshold-based, but either automate the choice of SUV threshold specific to an image [326,327] or apply thresholds to a combination (e.g., ratio) of SUV and an image-specific background value [328,329]. More segmentation algorithms are entering PET oncology from the field of computer vision [330] including the use of image gradients [320], deformable contour models [331,332], mutual information in hybrid images [333] and histogram mixture models for heterogeneous regions [334]. The current staging methods in oncology are based on the appraisal of size, number and location of the neoplastic lesions at baseline but they do not provide accurate measurement of tumor volume. The latter, in fact, is only loosely correlated to its size; as an example, patients presenting with micro-metastases in lymph nodes may show the same $\mathrm{N}$ value in TNM staging than patients with bulky nodal lesions [335]. Data available in patients affected by HNSCC, lung carcinoma, esophageal carcinoma and gynecological malignancies suggest that MTV and to a lesser extent TLG have the potential to become valuable in the imaging of human solid tumors as prognostic biomarkers for short- to intermediate-term survival outcomes, adding value to clinical staging, for assessment of response to treatment with neo-adjuvant and concurrent chemotherapy, and for treatment optimization [336]. Based on early treatment response assessment using changes in metabolic tumor volume over time, it might be possible to select patients who require a more aggressive treatment to improve their outcome.

TNM staging system is currently the most widely applied prognostic system for patients with HNSCC, but it proved suboptimal for identification of patients at high risk of recurrence [337]. Chung et al. studied 43 clinically node-negative patients with oral HNSCC who had all undergone primary tumor resection and elective neck dissection in addition to a pretreatment FDG-PET/CT examination [338]. In order to correlate the prognostic impact of imaging results and surgical staging the following parameters were evaluated: age, gender, clinical T stage, $\mathrm{SUV}_{\mathrm{Max}}$, MTV and depth of invasion, lympho-vascular invasion, pathological tumor volume and histological differentiation. In multivariate analysis, MTV proved the only relevant prognostic factor in predicting tumor spread (hazard ratio 54.66, CI 1.05-2842.86); patients with an MTV of $>6 \mathrm{~mL}$ had significantly higher numbers of occult metastases. Dibble et al. [339] reported the value of MTV and TLG in a cohort of 45 patients with histologically proven oral or oro-pharyngeal HNSCC staged at baseline with FDG-PET/CT. The only parameters significantly associated with survival and treatment outcome were MTV and TLG. The median cut-off point of $7.7 \mathrm{~mL}$ for primary tumor MTV was the best predictive value on receiver operator characteristics (ROC) analysis. MTV and TLG in primary tumor and of lymph nodes were prospectively assessed by Chan et al. [340] in a cohort of 196 stage III/IVb patients affected by HNSCC of the nasopharynx, Combination of a TLG $>330 \mathrm{~mL}$ and stage IV were 
predictive of local recurrence, while high $\mathrm{SUV}_{\mathrm{Max}}$ of lymph nodes and stage IV were predictive of distant disease spread.

In lung cancer, the FDG uptake intensity at baseline and after neo-adjuvant chemotherapy proved a powerful predictor of survival, and $\mathrm{SUV}_{\mathrm{Max}}$ overtook TNM staging in predicting chemotherapy response [336]. Therefore the prognostic value of $\mathrm{SUV}_{\mathrm{Max}}$ of the primary tumor in FDG-PET/CT studies for risk stratification in NSCLC patients has been increasingly used [341]. Lee et al. identified 19 patients with lung cancer (18 of them with NSCLC) staged and re-staged by FDG-PET/CT scans before and after therapy, and at the time of progression in 15 out of 19 experiencing tumor relapse [342]. Metabolically active tumor regions were segmented on pre- and post-treatment FDG-PET/CT scans semi automatically using custom software. Median MTV was $27 \mathrm{~mL}$ and, in multivariate analysis, an increase in MTV $\geq 25 \mathrm{~mL}$ in end of treatment was associated with increased hazard of progression and of death (5.4-fold and 7.6-fold), statistically significant ( $p=0.0014$ and $p=0.001$ ) after controlling for stage, treatment intent, age, Karnofsky performance status, and weight loss. In a comprehensive large review on 11 (10 retrospective, one prospective) studies for a total number of 1,204 patients suffering from lung cancer (955 with NSCLL and 249 SCLL) both in limited and extended disease, Van de Wiele et al. [336] found that MTV in nearly all studies and in lower proportion, TLG were predictive of disease free survival overall survival or macroscopic/microscopic disease spread.

Among patients affected by esophageal carcinoma most present with widespread disease and, in spite of improvements in surgical and neo-adjuvant therapies, die for their disease within 1 year from diagnosis $[343,344]$. Resectable disease and TNM stage at the time of diagnosis are by far the most important prognostic factors. Additional factors are tumor length, the number and the proportion of involved lymph nodes. Tumor resection is possible in stage I-III, but in stage III, due to the presence of a locally advanced disease tumor control with surgery alone is poor, and neo-adjuvant chemotherapy seems useful in prolonging time to progression.

Interim assessment of clinical response with FDG-PET/CT during neo-adjuvant treatment is required to permit early treatment adaptation [345,346]. To detect pathological responders from non-responders before surgical resection of locally advanced esophageal carcinoma, Jayachandran et al. calculated the predictive value of 0.5. In their series of 37 patients, both MTV2.5 and TLG2.5 (MTV and TLG defined using a fixed SUV threshold of 2.5) were predictive of response and survival, whereas $\mathrm{SUV}_{\text {Max }}$ was not [347]. It was suggested that FDG-PET/CT scan may potentially guide (surgical) therapy after chemo-radiotherapy. MTV assessment with FDG-PET/CT has been used to determine tumor length and locally advanced tumor bulk [348,349]. MTV2.5 (was also used in a study by Roedl et al. for metabolic tumor length determination on pre- and post-treatment scans after neo-adjuvant chemo-radiotherapy in a series of 47 patients, to check whether a tumor length decrease $\geq 33 \%$ was predictive of histopathological response [350]. Using this threshold, a sensitivity and specificity for predicting pathological response of $91 \%$ and $92 \%$, respectively, were obtained, versus $86 \%$ and $61 \%$ using a threshold $\geq 43 \% \mathrm{SUV}_{\mathrm{Max}}$ decrease from baseline values.

Between $20 \%$ and $40 \%$ of patients with cervix carcinoma of the uterus, treated with concurrent chemoradiation, will have recurrent or persistent disease [351]. As in other solid cancers the early identification of high-risk patients for treatment failure could pave the way to an intensified treatment for this patient subset. Kidd et al. have also shown in a series of 25 patients with stage Ib1-IVa 
cervical cancer scheduled to undergo chemoradiation that higher pretreatment MTV40\% values $\left(\geq 55.4 \mathrm{~cm}^{3}\right)$ are significantly associated with poor treatment response in multivariate analysis [352].

In conclusion, despite the heterogeneity in the clinical behavior and aggressiveness of the neoplastic disorders, the prognostic meaning of MTV and TLG persist across different types of solid cancer and seems more evident in locally-advanced tumors, where the risk of disease recurrence after surgery is highest and the benefits of neo-adjuvant chemotherapy or chemoradiation more evident. Since TNM stage is often used to decide the indication of neo-adjuvant chemotherapy, MTV showed higher predictive value and superseded anatomical tools such as TNM staging to guide preoperative treatment.

\section{FDG-PET and Multiple Myeloma}

Multiple Myeloma (MM) is a hematologic neoplasm characterized by bone marrow invasion of a neoplastic clone of plasma cells, presence of bone lytic lesions and extra-medullary organ invasion in later stages of the disease [353]. The disease course is heterogeneous with a patient survival ranging from a few months to more than a decade [354]. In this perspective a still unfilled need exists for reliable prognostic tool identification in order to predict the individual disease outcome in a single-patient basis. As in other solid and hematologic cancers one of the most important prognostic factors is tumor dissemination (or stage) at baseline; the most frequently involved organ is bone and bone marrow but, due to the heterogeneity of the pattern of bone and bone marrow invasion by disease standardization and grading of tumor spread in this organ has been difficult [355].

The MM staging system was introduced in 1975 by Durie and Salmon to allow patient stratification with different tumor bulk by calculating the measurable theoretical myeloma cell mass [356]. However, the emergence in the last decade of new imaging techniques, as MRI and FDG-PET/CT have provided new insights on tumor spread at disease onset and gradually revolutionized staging system developing a more integrating clinical and imaging approach, the Durie/Salmon PLUS myeloma staging [357]. Sensitivity of FDG-PET/CT proved very high for detection of extra-skeletal lesions, while MRI outperforms FDG-PET/CT for detecting bone and bone marrow invasion by disease, especially for spine and pelvic localizations [358].

FDG-PET/CT proved a powerful prognosticator in MM: MTV measurement at baseline, and longitudinal studies aimed at assessing the role of minimal residual disease detection by FDG-PET/CT are the most innovative aspect of functional imaging in this disease.

Durie and Salmon staging [356] and later on the International Staging System [355] have been proposed for tumor spread assessment, laying on a wide array of biochemical markers and conventional radiographic imaging for bone lytic lesion detection. However, a definite cutoff for serum albumin and beta2-microglobulin still lacks due to a frequently coexisting impaired renal function; furthermore, conventional radiography can significantly underestimate lytic lesions because up to $30 \%$ of the trabecular bone could be missed in standard radiographs. In this setting, a number of different diseases showing a wide range of tumor bulk and could be included in the same category of stage III MM.

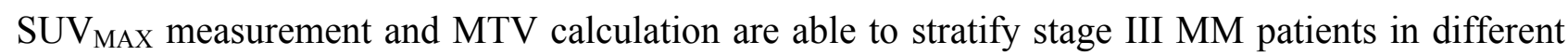
subsets according to tumor bulk, characterized by different prognosis and treatment outcome. These new insights on MM tumor burden originate from the pioneer retrospective study by Fonti et al. on the prognostic value of MTV assessed at baseline by FDG-PET/CT in a cohort of 47 stage IIIA MM 
patients treated with bortezomid and lenalidomide or thalidomide and autologous stem cell transplantation in half of the patients [359]. ROI for MTV calculations were defined as focal lesions detected on CT scan showing a $\mathrm{SUV}_{\text {Max }} \geq 2.5$ on PET. MTV was calculated using an automated contouring program based on a fixed threshold value of $40 \%$ of $\mathrm{SUV}_{\mathrm{Max}}$. At follow-up, patients who developed progressive disease or dying for myeloma showed a significantly higher MTV (74.7 \pm 19.3 vs. $29.8 \pm 5.1 \mathrm{~mL}, p=0.009$ and $123.2 \pm 29.8 \pm 4.2, p=0.0001)$. PFS was significantly prolonged in patients with MTV $<42.2 \mathrm{~mL}$ as compared with patients with MTV $\geq 42.2 \mathrm{~mL}(p=0.0465)$ and OS was significantly better in patients with MTV $<77.6 \mathrm{~mL}$ as compared had MTV $\geq 77.6 \mathrm{~mL}$. In conclusion MTV seems to be closely correlated to plasma cell mass and may be a valid clinical help to correlate with patients outcome.

Zamagni et al. analyzed the prognostic relevance of FDG-PET/CT on treatment outcome in a longitudinal prospective study [358]. In a cohort of $192 \mathrm{MM}$ patients consecutively enrolled, FDG-PET/CT was performed at diagnosis, after induction therapy with thalidomide and dexametasone (TD), after double autologous stem cell transplantation (ASCT) and later during follow-up. Presence at baseline of at least three focal lesions (44\% of cases), a $\mathrm{SUV}_{\mathrm{Max}}>4.2$ (46\%), and extra-medullary disease (EMD; 6\%) adversely affected 4-year estimates of progression-free survival (PFS; $>3$ FLs: 50\%; SUV > 4.2: 43\%; presence of EMD: $28 \%$ ). $\mathrm{SUV}_{\mathrm{Max}}>4.2$ and EMD were also correlated with shorter overall survival (OS; 4-year rates: $77 \%$ and $66 \%$, respectively). Patients with a negative baseline FDG-PET/CT were 24\% and positive 76\%. A diffuse bone marrow FDG uptake was observed in $17 \%$ while $59 \%$ had focal lesions with an $\mathrm{SUV}_{\mathrm{Max}}>4.2$ in $46 \%$ and $\mathrm{SUV}<$ or equal 4.2 in $54 \%$. This threshold of 4.2 in SUV could distinguish a 4-year PFS of $4.2 \%$ and $66 \%$, respectively $(p=0.003)$ and 4 -year OS of $76 \%$ and $92 \%$, respectively $(p=0.02)$. After induction FDG-PET/CT-positive patients were $63 \%$, the same $\mathrm{SUV}_{\mathrm{Max}}$ threshold lower of higher than 4.2 was able to distinguish two distinct patient cohorts with a a 4 -year PFS of $44 \%$ and $69 \%$, respectively $(p=0.007)$ and a 4 -year OS of $75 \%$ and $88 \%$, respectively $(p=0.09)$. After ASCT 4-year PFS and OS for FDG-PET/CT-negative patients were $47 \%$ and $79 \%$, respectively, compared with values of $32 \%(p=0.02)$ and $66 \%(p=0.02)$, respectively, for FDG-PET/CT-positive patients.

In conclusion, with the limitations intrinsic to a quantitative analysis of FDG-PET/CT data in absence of information on scanner calibrations, image acquisition and reconstruction, and fasting glucose levels of the scanned patients, and of a retrospective nature of the study, this preliminary report seems to stress the relevant prognostic role of FDG-PET/CT throughout the during the natural history of the disease and could pave the way to prospective studies aimed at assessing a patient-adapted strategy for MM treatment.

\section{FDG-PET/CT in Surveillance of Cancer}

At this time, FDG-PET/CT is not routinely recommended in the surveillance of most cancers [360,361]. Actually, there is insufficient evidence to define the clinical impact of FDG-PET/CT in this indication [362]. This is the case for lymphoma, for which several studies observed a low PPV of FDG-PET/CT, with a high rate of false-positive results [363]. However, some data suggest that FDG-PET/CT may be interesting in surveillance and follow-up; this is the case of a prospective study of 91 HNSCC patients, which observed a high effectiveness of FGD-PET/CT in the assessment of the 
disease recurrence (sensitivity 100\%, specificity 85\%, PPV 77\%, NPV 100\%). In this study, FDG-ET/CT examination was performed $11.6 \pm 4.4$ months after the end of the treatment. In contrast, a recent metaanalysis observed more various rates of sensitivity and PPV (75\%-100\% and 50\%-90\%, respectively) for the surveillance of head and neck cancer [362]. For colorectal cancer, a randomized controlled trial of 130 patients reported that recurrences were detected after a shorter time (12.1 vs. 15.4 months; $p=0.01)$ in the PET group compared to the conventional group, and recurrences were also more frequently (10 vs. two patients) cured by surgery in the PET group [364]. The authors concluded that FDG-PET/CT may permit the earlier detection of recurrence of colorectal cancer, but once again, more data are warranted to confirm that. The potential risks of using FDG-PET/CT for surveillance are overtreatment caused by false-positives, and unnecessary radiation exposure. The lack of a common definition of surveillance (the minimal time since last treatment, the absence of clinical or other diagnostic suspicion of recurrence) is also an unsolved issue at this time [362]. In the case of surveillance of melanoma, the data are clearer [129], and FDG-PET/CT is a promising tool in this field. Finally, we should remember that FDG-PET/CT is a useful imaging modality in case of elevation of serum tumor markers, in particular in ovarian cancer [97].

\section{New PET Technologies and Tracers}

FDG is the most frequently used PET radiopharmaceutical, but other PET tracers are progressively developed and used, not only in literature publications, but also in clinical practice [365]. We will expose some example to illustrate this topic.

The main application of ${ }^{18} \mathrm{~F}$-fluorocholine $(\mathrm{FCH})$ and ${ }^{11} \mathrm{C}$-fluorocholine, some phospholipid cell membrane metabolism markers, is prostate cancer. FDG is also often suboptimal in prostate cancer, because of low tracer avidity, and FCH-PET/CT is a promising alternative molecular imaging in that field [367]. Its main indication is prostate-specific antigen (PSA) elevation, which has been demonstrated as a useful issue in several publications (pooled sensitivity of $85.6 \%$ and pooled specificity of $92.6 \%$ in the meta-analysis of Evangelista et al.) [367,368]. Due to the strong relationship between PSA kinetics and detection rate of FCH-PET/CT, it should be taken into account in the selection of prostate cancer patients who should undergo FCH-PET/CT for restaging [369]. A recent meta-analysis observed that FCH-PET/CT led to a change in treatment in 381 (41\%) of 938 patients who performed FCH-PET/CT during staging and restaging for biochemical recurrence [368]. However, in staging of patients with proven but untreated, high-risk prostate cancer patients, there is limited but promising evidence warranting further studies; in this setting, Choline-PET/CT has a pooled sensitivity and specificity of 84\% (95\% confidence interval: 68\%-93\%) and 79\% (95\% CI, 53\%-93\%), respectively [370], and provides low sensitivity in the detection of lymph node metastases prior to surgery (pooled sensitivity 49.2\%) [371].

For brain imaging, FDG uptake correlates with tumor grade, cell density, biological aggressiveness and survival in patients with primary or recurrent gliomas [372]. However, this approach can be limited due to the high physiological cerebral activity. Dual time point imaging which takes advantage of the slower dephosphorylation of tumoral FDG vs. normal brain improves tumor to normal brain contrast [373,374]. Fluorinated amino acids, 18F-fluoroethyltyrosine (FET) and 18F-Fluorodihydroxyphenylalanine $\left({ }^{18} \mathrm{~F}\right.$-DOPA) are an interesting alternative for brain tumor imaging 
due to the specific over-expression of amino-acids transporters in brain tumors unlike normal brain tissue. Both of them show comparable results to 11C-methionine PET and are able to help diagnosing tumor recurrence versus radiation necrosis, guiding stereotactic biopsy and treatment evaluation [375-377]. They are also useful in brain metastases evaluation [378]. Relative differences are still debated especially concerning low-grade gliomas evaluations [379].

${ }^{18} \mathrm{~F}$-DOPA, the immediate precursor of dopamine, has also high diagnostic performances in adrenal and extra-adrenal paragangliomas [380] or in the detection of recurrent medullary thyroid carcinoma, and may be an interesting alternative imaging modality in neuroendocrine tumors [381].

Other tracers like ${ }^{18} \mathrm{~F}$-fluorothymidine (FLT), a thymidine analogs, explore the cell proliferation and can be used to detect tumors in many areas of the body. We have to take into account that background uptake of FLT is high in the liver, marrow, and renal system, limiting use in these organs; actually, its most promising use is in monitoring tumor treatment response [382,383].

More specific tracers may also be used, like ${ }^{18} \mathrm{~F}$-fluoride, an osseous marker which has higher image quality than SPECT-CT bone scintigraphy equivalent [365]. According to the SNM Practice Guideline for Sodium ${ }^{18} \mathrm{~F}$-Fluoride PET/CT Bone Scans 1.0, PET/CT ${ }^{18} \mathrm{~F}$-fluoride bone scans may be used to identify skeletal metastases, including localization and determination of the extent of disease [384].

Other specific radiopharmaceuticals exist, like ${ }^{124}$ I-Iodine for PET/CT imaging of thyroid cancer, ${ }^{18} \mathrm{~F}$-fluoroestradiol for imaging of estrogen receptors in breast cancer, or ${ }^{68} \mathrm{Ga}$-labelled somatostatin analogue [365].

The main application of ${ }^{68} \mathrm{Ga}$ generator is the exploration of somatostatin receptors. ${ }^{68} \mathrm{Ga}$-labelled somatostatin analogue PET/CT is useful for the staging of neuroendocrine tumors (gastro-entero-pancreatic or bronchial neuroendocrine tumors mainly), and seems to be superior compared to ${ }^{111}$ In-DTPA-octreotide SPECT-CT [385,386]. This radiopharmaceutical may provide additional diagnostic information in a high proportion of patients with consequent high management impact. ${ }^{68}$ Ga-labelled somatostatin analogue PET/CT could replace ${ }^{111}$ In-DTPA-octreotide scintigraphy at centers where it is available given its superior accuracy, faster acquisition and lower radiation exposure. Moreover, it offers the possibility to noninvasively evaluate neuroendocrine tumor cells for the presence of somatostatin receptor expression, with direct therapeutic implications [387].

In addition to new radiopharmaceuticals, new PET technologies, mainly FDG-PET/MRI, may offer new opportunities. Hybrid FDG-PET/MRI may be particularly interesting because of its superior resolution and soft tissue contrast (e.g., tumors in the brain, the head-and-neck region, or the pelvis), and its lack of ionizing radiation exposure [388]. Recent studies show the effectiveness of whole-body FDG-PET/MRI imaging in oncology, observing that FDG-PET/MRI performed comparatively to FDG-PET/CT in lesion detection and quantitative measurements [389,390]. Several potential applications are described in the literature: the evaluation of bone metastases from prostate cancer with simultaneous FCH-PET/MRI [391], the follow-up of head and neck cancer patients [392], the initial staging of pediatric lymphoma as a radiation-free alternative to FDG-PET/CT [393], or the lung cancer M-staging especially for brain and liver metastases [394]. 


\section{Conclusions}

During the last 20 years the continuous technological progress has revolutionized the role of PET scan in Oncology. Moving from its original role for tumor staging and restaging PET/CT has become a seminal tool for tumor prognostication: as a new metrics for tumor volume and spread measurement at baseline and as a compass for treatment tailoring both in lymphoma and solid tumors like esophageal carcinoma, inoperable non-small cell lung carcinoma and metastatic breast cancer. Challenges for its use with nuclear magnetic resonance in a single-shot scan, and as a unit of measure for tumor burden quantification are opening new frontiers for the medical imaging research.

\section{Abbreviations}

\begin{tabular}{|c|c|}
\hline FDG & 18-Fluorodeoxyglucose \\
\hline PET & Positron Emission Tomography \\
\hline $\mathrm{CT}$ & Computed Tomography \\
\hline FDG-PET/CT & Combined Positron Emission Tomography and Computed Tomography \\
\hline SPECT & Single-photon Emission Computed Tomography \\
\hline MRI & Magnetic Resonance Imaging \\
\hline US & Ultrasound \\
\hline EUS & Endoscopy Ultrasound \\
\hline BS & Bone Scintigraphy \\
\hline SUV & Standardized Uptake Value \\
\hline $\mathrm{SUV}_{\mathrm{Max}}$ & Maximal SUV Value \\
\hline $\mathrm{SUV}_{\text {Mean }}$ & Median SUV Value \\
\hline SUV $V_{\text {Peak }}$ & SUV value in a1 $-\mathrm{cm}^{3}$ volume spherical ROI \\
\hline$\Delta \mathrm{SUV}$ & reduction in $\mathrm{SUV}_{\mathrm{Max}}$ \\
\hline ROI & Region Of Interest \\
\hline MTV & Metabolic Tumor Volume \\
\hline TLG & Total Lesion Glycolysis \\
\hline $\mathrm{CeCT}$ & Contrast-Enhanced Computed Tomography \\
\hline BMB & Bone Marrow Biopsy \\
\hline $\mathrm{B} / \mathrm{BM}$ & Bone and Bone Marrow \\
\hline PPV & Positive Predictive Value \\
\hline NPV & Negative Predictive Value \\
\hline AUC & Area Under Curve \\
\hline ROC & Receiver Operating Charateristic \\
\hline
\end{tabular}

\section{Conflicts of Interest}

The authors declare no conflict of interest. 


\section{References}

1. Avril, N.; Sassen, S.; Roylance, R. Response to therapy in breast cancer. J. Nucl. Med. 2009, $50,55 \mathrm{~S}-63 \mathrm{~S}$.

2. Strauss, L.G.; Conti, P.S. The applications of PET in clinical oncology. J. Nucl. Med. 1991, 32, 623-648.

3. Zasadny, K.R.; Wahl, R.L. Standardized uptake values of normal tissues at PET with 2-[fluorine18]-fluoro-2-deoxy-D-glucose: Variations with body weight and a method for correction. Radiology 1993, 189, 847-850.

4. American Joint Committee on Cancer. AJCC Cancer Staging Manual, 6th ed.; Springer: New York, NY, USA, 2002.

5. Adebonojo, S.A.; Bowser, A.N.; Moritz, D.M.; Corcoran, P.C. Impact of revised stage classification of lung cancer on survival: A military experience. Chest 1999, 115, 1507-1513.

6. Mountain, C.F. Revisions in the International System for Staging Lung Cancer. Chest 1997, 111, $1710-1717$.

7. Tylski, P.; Stute, S.; Grotus, N.; Doyeux, K.; Hapdey, S.; Gardin, I.; Vanderlinden, B.; Buvat, I. Comparative assessment of methods for estimating tumor volume and standardized uptake value in (18)F-FDG PET. J. Nucl. Med. 2010, 51, 268-276.

8. Larson, S.M.; Erdi, Y.; Akhurst, T.; Mazumdar, M.; Macapinlac, H.A.; Finn, R.D.; Casilla, C.; Fazzari M.; Srivastava N.; Yeung, H.W. Tumor Treatment Response Based on Visual and Quantitative Changes in Global Tumor Glycolysis Using PET-FDG Imaging. The Visual Response Score and the Change in Total Lesion Glycolysis. Clin. Positron Imaging 1999, 2, 159-171.

9. Obara, P.; Pu, Y. Prognostic value of metabolic tumor burden in lung cancer. Chin. J. Cancer Res. (Chung-Kuo Yen Cheng Yen Chiu) 2013, 25, 615-622.

10. Weng, E.; Tran, L.; Rege, S.; Safa, A.; Sadeghi, A.; Juillard, G.; Mark, R.; Santiago, S.; Brown, C.; Mandelkern, M. Accuracy and clinical impact of mediastinal lymph node staging with FDG-PET imaging in potentially resectable lung cancer. Am. J. Clin. Oncol. 2000, 23, 47-52.

11. Changlai, S.P.; Tsai, S.C.; Chou, M.C.; Ho, Y.J.; Kao, C.H. Whole body 18F-2-deoxyglucose positron emission tomography to restage non-small cell lung cancer. Oncol. Rep. 2001, 8, 337-339.

12. Taus, A.; Aguilo, R.; Curull, V.; Suarez-Pinera, M.; Rodriguez-Fuster, A.; Rodriguez de Dios, N.; Zuccarino, F.; Vollmer, I.; Sánchez-Font, A.; Belda-Sanchis, J.; et al. Impact of 18F-FDG PET/CT in the Treatment of Patients With Non-Small Cell Lung Cancer. Arch. Bronconeumol. 2014, 50, 99-104.

13. Maziak, D.E.; Darling, G.E.; Inculet, R.I.; Gulenchyn, K.Y.; Driedger, A.A.; Ung, Y.C.; Miller, J.D.; Gu, C.S.; Cline, K.J.; Evans, W.K.; et al. Positron emission tomography in staging early lung cancer: A randomized trial. Ann. Internal Med. 2009, 151, 221-228, W-48.

14. Fischer, B.; Lassen, U.; Mortensen, J.; Larsen, S.; Loft, A.; Bertelsen, A.; Ravn, J.; Clementsen, P.; Høgholm, A.; Larsen, K.; et al. Preoperative staging of lung cancer with combined PET-CT. N. Engl. J. Med. 2009, 361, 32-39.

15. Imai, K.; Minamiya, Y.; Saito, H.; Motoyama, S.; Sato, Y.; Ito, A.; Yoshino, K.; Kudo, S.; Takashima, S.; Kawaharada, Y.; et al. Diagnostic imaging in the preoperative management of lung cancer. Surg. Today 2014, 44, doi:10.1007/s00595-013-0660-z. 
16. De Wever, W.; Ceyssens, S.; Mortelmans, L.; Stroobants, S.; Marchal, G.; Bogaert, J.; Verschakelen, J.A. Additional value of PET-CT in the staging of lung cancer: Comparison with CT alone, PET alone and visual correlation of PET and CT. Eur. Radiol. 2007, 17, 23-32.

17. Chao, F.; Zhang, H. PET/CT in the staging of the non-small-cell lung cancer. J. Biomed. Biotechnol. 2012, 2012, 783739.

18. Shim, S.S.; Lee, K.S.; Kim, B.T.; Chung, M.J.; Lee, E.J.; Han, J.; Choi, J.Y.; Kwon, O.J.; Shim, Y.M.; Kim, S. Non-small cell lung cancer: Prospective comparison of integrated FDG PET/CT and CT alone for preoperative staging. Radiology 2005, 236, 1011-1019.

19. Bille, A.; Pelosi, E.; Skanjeti, A.; Arena, V.; Errico, L.; Borasio, P.; Mancini, M.; Ardissone, F. Preoperative intrathoracic lymph node staging in patients with non-small-cell lung cancer: Accuracy of integrated positron emission tomography and computed tomography. Eur. J. CardioThoracic Surg. 2009, 36, 440-445.

20. Li, S.; Zheng, Q.; Ma, Y.; Wang, Y.; Feng, Y.; Zhao, B.; Yang, Y. Implications of False Negative and False Positive Diagnosis in Lymph Node Staging of NSCLC by Means of (18)FFDG PET/CT. PLoS One 2013, 8, e78552.

21. Tandberg, D.J.; Gee, N.G.; Chino, J.P.; D’Amico, T.A.; Ready, N.E.; Coleman, R.E.; Kelsey, C.R. Are discordant positron emission tomography and pathological assessments of the mediastinum in non-small cell lung cancer significant? J. Thoracic Cardiovasc. Surg. 2013, 146, 796-801.

22. Adams, K.; Shah, P.L.; Edmonds, L.; Lim, E. Test performance of endobronchial ultrasound and transbronchial needle aspiration biopsy for mediastinal staging in patients with lung cancer: Systematic review and meta-analysis. Thorax 2009, 64, 757-762.

23. Eschmann, S.M.; Friedel, G.; Paulsen, F.; Budach, W.; Harer-Mouline, C.; Dohmen, B.M.; Bares, R. FDG PET for staging of advanced non-small cell lung cancer prior to neoadjuvant radio-chemotherapy. Eur. J. Nucl. Med. Mol. Imaging 2002, 29, 804-808.

24. Seltzer, M.A.; Yap, C.S.; Silverman, D.H.; Meta, J.; Schiepers, C.; Phelps, M.E.; Gambhir, SS.; Rao, J.; Valk, P.E.; Czernin, J. The impact of PET on the management of lung cancer: The referring physician's perspective. J. Nucl. Med. 2002, 43, 752-756.

25. Erasmus, J.J.; Patz, E.F., Jr.; McAdams, H.P.; Murray, J.G.; Herndon, J.; Coleman, R.E. Goodman, P.C. Evaluation of adrenal masses in patients with bronchogenic carcinoma using 18F-fluorodeoxyglucose positron emission tomography. AJR Am. J. Roentgenol. 1997, 168, $1357-1360$.

26. Stone, W.Z.; Wymer, D.C.; Canales, B.K. Fluorodeoxyglucose-positron-emission tomography/ computed tomography imaging for adrenal masses in patients with lung cancer: Review and diagnostic algorithm. J. Endourol. 2014, 28, 104-111.

27. DiPerna, C.A.; Wood, D.E. Surgical management of T3 and T4 lung cancer. Clin. Cancer Res. 2005, 11, 5038s-5044s.

28. Tagliabue, L. The emerging role of FDG PET/CT in rectal cancer management: Is it time to use the technique for early prognostication? Eur. J. Nucl. Med. Mol. Imaging 2013, 40, 652-656.

29. NCCN version 4.2013. Available online: http://www.NCCN.org (accessed on 25 July 2014).

30. Kekelidze, M.; D’Errico, L.; Pansini, M.; Tyndall, A.; Hohmann, J. Colorectal cancer: Current imaging methods and future perspectives for the diagnosis, staging and therapeutic response evaluation. World J. Gastroenterol. 2013, 19, 8502-8514. 
31. Bipat, S.; Niekel, M.C.; Comans, E.F.; Nio, C.Y.; Bemelman, W.A.; Verhoef, C.; Stoker, J. Imaging modalities for the staging of patients with colorectal cancer. Neth. J. Med. 2012, 70, 26-34.

32. Tsunoda, Y.; Ito, M.; Fujii, H.; Kuwano, H.; Saito, N. Preoperative diagnosis of lymph node metastases of colorectal cancer by FDG-PET/CT. Jpn. J. Clin. Oncol. 2008, 38, 347-353.

33. Niekel, M.C.; Bipat, S.; Stoker, J. Diagnostic imaging of colorectal liver metastases with CT, MR imaging, FDG PET, and/or FDG PET/CT: A meta-analysis of prospective studies including patients who have not previously undergone treatment. Radiology 2010, 257, 674-684.

34. Bamba, Y.; Itabashi, M.; Kameoka, S. Value of PET/CT imaging for diagnosing pulmonary metastasis of colorectal cancer. Hepatogastroenterology 2011, 58, 1972-1974.

35. Briggs, R.H.; Chowdhury, F.U.; Lodge, J.P.; Scarsbrook, A.F. Clinical impact of FDG PET-CT in patients with potentially operable metastatic colorectal cancer. Clin. Radiol. 2011, 66, 1167-1174.

36. Kochhar, R.; Liong, S.; Manoharan, P. The role of FDG PET/CT in patients with colorectal cancer metastases. Cancer Biomarkers 2010, 7, 235-248.

37. Grassetto, G.; Fornasiero, A.; Bonciarelli, G.; Banti, E.; Rampin, L.; Marzola, M.C.; Massaro, A; Galeotti, F.; del Favero, G.; Pasini, F. Additional value of FDG-PET/CT in management of "solitary" liver metastases: Preliminary results of a prospective multicenter study. Mol. Imaging Biol. 2010, 12, 139-144.

38. Bonanni, L.; De'liguori Carino, N.; Deshpande, R.; Ammori, B.J.; Sherlock, D.J.; Valle, J.W.; Tam, E.; O’Reilly, D.A. A comparison of diagnostic imaging modalities for colorectal liver metastases. Eur. J. Surg. Oncol. 2014, 40, 545-550.

39. Brush, J.; Boyd, K.; Chappell, F.; Crawford, F.; Dozier, M.; Fenwick, E.; Glanville, J.; McIntosh, H.; Renehan, A.; Weller, D.; et al. The value of FDG positron emission tomography/computerised tomography $(\mathrm{PET} / \mathrm{CT})$ in pre-operative staging of colorectal cancer: A systematic review and economic evaluation. Health Technol. Assess. 2011, 15, 1-192, iii-iv.

40. Lutz, M.P.; Zalcberg, J.R.; Ducreux, M.; Ajani, J.A.; Allum, W.; Aust, D.; Bang, Y.J.; Cascinu, S.; Hölscher, A.; Jankowski, J.; et al. Highlights of the EORTC St. Gallen International Expert Consensus on the primary therapy of gastric, gastroesophageal and oesophageal cancerDifferential treatment strategies for subtypes of early gastroesophageal cancer. Eur. J. Cancer 2012, 48, 2941-2953.

41. Tangoku, A.; Yamamoto, Y.; Furukita, Y.; Goto, M.; Morimoto, M. The new era of staging as a key for an appropriate treatment for esophageal cancer. Ann. Thoracic Cardiovasc. Surg. 2012, 18, 190-199.

42. Karaosmanoglu, A.D.; Blake, M.A. Applications of PET-CT in patients with esophageal cancer. Diagn. Int. Radiol. 2012, 18, 171-182.

43. Okada, M.; Murakami, T.; Kumano, S.; Kuwabara, M.; Shimono, T.; Hosono, M.; Shiozaki, H. Integrated FDG-PET/CT compared with intravenous contrast-enhanced CT for evaluation of metastatic regional lymph nodes in patients with resectable early stage esophageal cancer. Ann. Nucl. Med. 2009, 23, 73-80.

44. Vazquez-Sequeiros, E.; Foruny-Olcina, J.R. Linear EUS: The clinical impact of $\mathrm{N}$ staging in esophageal carcinoma. Minerva Med. 2007, 98, 313-319.

45. Shi, W.; Wang, W.; Wang, J.; Cheng, H.; Huo, X. Meta-analysis of 18FDG PET-CT for nodal staging in patients with esophageal cancer. Surg. Oncol. 2013, 22, 112-116. 
46. Manabe, O.; Hattori, N.; Hirata, K.; Itoh, K.; Hosokawa, M.; Takahashi, H.; Oyama-Manabe, N.; Tamaki, N. Diagnostic accuracy of lymph node metastasis depends on metabolic activity of the primary lesion in thoracic squamous esophageal cancer. J. Nucl. Med. 2013, 54, 670-676.

47. Van Vliet, E.P.; Heijenbrok-Kal, M.H.; Hunink, M.G.; Kuipers, E.J.; Siersema, P.D. Staging investigations for oesophageal cancer: A meta-analysis. Br. J. Cancer 2008, 98, 547-557.

48. Hsu, P.K.; Lin, K.H.; Wang, S.J.; Huang, C.S.; Wu, Y.C.; Hsu, W.H. Preoperative positron emission tomography/computed tomography predicts advanced lymph node metastasis in esophageal squamous cell carcinoma patients. World J. Surg. 2011, 35, 1321-1326.

49. Barber, T.W.; Duong, C.P.; Leong, T.; Bressel, M.; Drummond, E.G.; Hicks, R.J. 18F-FDG PET/CT has a high impact on patient management and provides powerful prognostic stratification in the primary staging of esophageal cancer: A prospective study with mature survival data. J. Nucl. Med. 2012, 53, 864-871.

50. Sun, G.; Tian, J.; Gorospe, E.C.; Johnson, G.B.; Hunt, C.H.; Lutzke, L.S.; Leggett, C.L.; Iyer, P.G.; Wang, K.K. Utility of baseline positron emission tomography with computed tomography for predicting endoscopic resectability and survival outcomes in patients with early esophageal adenocarcinoma. J. Gastroenterol. Hepatol. 2013, 28, 975-981.

51. Chan, D.S.; Fielding, P.; Roberts, S.A.; Reid, T.D.; Ellis-Owen, R.; Lewis, W.G. Prognostic significance of 18-FDG PET/CT and EUS-defined tumour characteristics in patients with oesophageal cancer. Clin. Radiol. 2013, 68, 352-357.

52. Shum, W.Y.; Ding, H.J.; Liang, J.A.; Yen, K.Y.; Chen, S.W.; Kao, C.H. Use of pretreatment metabolic tumor volumes on PET-CT to predict the survival of patients with squamous cell carcinoma of esophagus treated by curative surgery. Anticancer Res. 2012, 32, 4163-4168.

53. Sehdev, A.; Catenacci, D.V. Gastroesophageal cancer: Focus on epidemiology, classification, and staging. Discov. Med. 2013, 16, 103-111.

54. Stahl, A.; Ott, K.; Weber, W.A.; Becker, K.; Link, T.; Siewert, J.R.; Schwaiger, M.; Fink, U.; FDG PET imaging of locally advanced gastric carcinomas: Correlation with endoscopic and histopathological findings. Eur. J. Nucl. Med. Mol. Imaging 2003, 30, 288-295.

55. Nath, J.; Moorthy, K.; Taniere, P.; Hallissey, M.; Alderson, D. Peritoneal lavage cytology in patients with oesophagogastric adenocarcinoma. Br. J. Surg. 2008, 95, 721-726.

56. Dibble, E.H.; Karantanis, D.; Mercier, G.; Peller, P.J.; Kachnic, L.A.; Subramaniam, R.M. PET/CT of cancer patients: Part 1, pancreatic neoplasms. AJR Am. J. Roentgenol. 2012, 199, 952-967.

57. Wang, Z.; Chen, J.Q.; Liu, J.L.; Qin, X.G.; Huang, Y. FDG-PET in diagnosis, staging and prognosis of pancreatic carcinoma: A meta-analysis. World J. Gastroenterol. 2013, 19, 4808-4817.

58. Hu, S.L.; Yang, Z.Y.; Zhou, Z.R.; Yu, X.J.; Ping, B.; Zhang, Y.J. Role of SUV(max) obtained by 18F-FDG PET/CT in patients with a solitary pancreatic lesion: Predicting malignant potential and proliferation. Nucl. Med. Commun. 2013, 34, 533-539.

59. Choi, H.J.; Kang, C.M.; Lee, W.J.; Song, S.Y.; Cho, A.; Yun, M.; Lee, J.D.; Kim, J.H.; Lee, J.H. Prognostic value of $18 \mathrm{~F}$-fluorodeoxyglucose positron emission tomography in patients with resectable pancreatic cancer. Yonsei Med. J. 2013, 54, 1377-1383. 
60. Moon, S.Y.; Joo, K.R.; So, Y.R.; Lim, J.U.; Cha, J.M.; Shin, H.P.; Yang, Y.J. Predictive value of maximum standardized uptake value (SUVmax) on 18F-FDG PET/CT in patients with locally advanced or metastatic pancreatic cancer. Clin. Nucl. Med. 2013, 38, 778-783.

61. Xu, H.X.; Chen, T.; Wang, W.Q.; Wu, C.T.; Liu, C.; Long, J.; Xu, J.; Zhang,Y.J.; Chen, R.H.; Liu, L.; et al. Metabolic tumour burden assessed by F-FDG PET/CT associated with serum CA19-9 predicts pancreatic cancer outcome after resection. Eur. J. Nucl. Med. Mol. Imaging 2014, 41, 1093-1102.

62. Kim, Y.I.; Kim, S.K.; Paeng, J.C.; Lee, H.Y. Comparison of F-18-FDG PET/CT findings between pancreatic solid pseudopapillary tumor and pancreatic ductal adenocarcinoma. Eur. J. Radiol. 2014, 83, 231-235.

63. Kato, K.; Nihashi, T.; Ikeda, M.; Abe, S.; Iwano, S.; Itoh, S.; Shimamoto, K.; Naganawa, S. Limited efficacy of (18)F-FDG PET/CT for differentiation between metastasis-free pancreatic cancer and mass-forming pancreatitis. Clin. Nucl. Med. 2013, 38, 417-421.

64. Matsumoto, I.; Shirakawa, S.; Shinzeki, M.; Asari, S.; Goto, T.; Ajiki, T.; Fukumoto, T.; Kitajima, K.; Ku, Y. 18-Fluorodeoxyglucose positron emission tomography does not aid in diagnosis of pancreatic ductal adenocarcinoma. Clin. Gastroenterol. Hepatol. 2013, 11, 712-718.

65. Nagamachi, S.; Nishii, R.; Wakamatsu, H.; Mizutani, Y.; Kiyohara, S.; Fujita, S.; Futami, S.; Sakae, T.; Furukoji, E.; Tamura, S. The usefulness of (18)F-FDG PET/MRI fusion image in diagnosing pancreatic tumor: Comparison with (18)F-FDG PET/CT. Ann. Nucl. Med. 2013, 27, 554-563.

66. Baiocchi, G.L.; Bertagna, F.; Gheza, F.; Grazioli, L.; Calanducci, D.; Giubbini, R.; Giubbini, R.; Portolani, N.; Giulini, S.M. Searching for indicators of malignancy in pancreatic intraductal papillary mucinous neoplasms: The value of 18FDG-PET confirmed. Ann. Surg. Oncol. 2012, 19, 3574-3580.

67. Bertagna, F.; Treglia, G.; Baiocchi, G.L.; Giubbini, R. F18-FDG-PET/CT for evaluation of intraductal papillary mucinous neoplasms (IPMN): A review of the literature. Jpn. J. Radiol. 2013, 31, 229-236.

68. Javery, O.; Shyn, P.; Mortele, K. FDG PET or PET/CT in patients with pancreatic cancer: When does it add to diagnostic CT or MRI? Clin. Imaging 2013, 37, 295-301.

69. Topkan, E.; Parlak, C.; Yapar, A.F. FDG-PET/CT-based restaging may alter initial management decisions and clinical outcomes in patients with locally advanced pancreatic carcinoma planned to undergo chemoradiotherapy. Cancer Imaging 2013, 13, 423-428.

70. Pauwels, E.K.; Coumou, A.W.; Kostkiewicz, M.; Kairemo, K. [ ${ }^{18}$ F]fluoro-2-deoxy-d-glucose positron emission tomography/computed tomography imaging in oncology: Initial staging and evaluation of cancer therapy. Med. Princ. Pract. 2013, 22, 427-437.

71. Haerle, S.K.; Strobel, K.; Ahmad, N.; Soltermann, A.; Schmid, D.T.; Stoeckli, S.J. Contrast-enhanced ${ }^{18}$ F-FDG-PET/CT for the assessment of necrotic lymph node metastases. Head Neck 2011, 33, 324-329.

72. Carlson, E.R.; Schaefferkoetter, J.; Townsend, D.; McCoy, J.M.; Campbell, P.D., Jr.; Long, M. The use of multiple time point dynamic positron emission tomography/computed tomography in patients with oral/head and neck cancer does not predictably identify metastatic cervical lymph nodes. J. Oral Maxillofac. Surg. 2013, 71, 162-177. 
73. Connell, C.A.; Corry, J.; Milner, A.D.; Hogg, A.; Hicks, R.J.; Rischin, D.; Peters, L.J. Clinical impact of, and prognostic stratification by, F-18 FDG PET/CT in head and neck mucosal squamous cell carcinoma. Head Neck 2007, 29, 986-995.

74. Roh, J.L.; Yeo, N.K.; Kim, J.S.; Lee, J.H.; Cho, K.J.; Choi, S.H.; Nam, S.Y.; Kim, S.Y. Utility of $2-[18 \mathrm{~F}]$ fluoro-2-deoxy-D-glucose positron emission tomography and positron emission tomography/computed tomography imaging in the preoperative staging of head and neck squamous cell carcinoma. Oral Oncol. 2007, 43, 887-893.

75. Gao, S.; Li, S.; Yang, X.; Tang, Q. FDG PET-CT for distant metastases in patients with recurrent head and neck cancer after definitive treatment. A meta-analysis. Oral Oncol. 2013, 50, 163-167.

76. Xu, G.Z.; Guan, D.J.; He, Z.Y. (18)FDG-PET/CT for detecting distant metastases and second primary cancers in patients with head and neck cancer. A meta-analysis. Oral Oncol. 2011, 47, $560-565$.

77. Bourgeois, A.C.; Warren, L.A.; Chang, T.T.; Embry, S.; Hudson, K.; Bradley, Y.C. Role of positron emission tomography/computed tomography in breast cancer. Radiol. Clin. N. Am. 2013, 51, 781-798.

78. Garami, Z.; Hascsi, Z.; Varga, J.; Dinya, T.; Tanyi, M.; Garai, I.; Damjanovich, L.; Galuska, L. The value of 18-FDG PET/CT in early-stage breast cancer compared to traditional diagnostic modalities with an emphasis on changes in disease stage designation and treatment plan. Eur. J. Surg. Oncol. 2012, 38, 31-37.

79. Bernsdorf, M.; Berthelsen, A.K.; Wielenga, V.T.; Kroman, N.; Teilum, D.; Binderup, T.; Tange, U.B.; Andersson, M.; Kjær, A.; Loft, A.; et al. Preoperative PET/CT in early-stage breast cancer. Ann. Oncol. 2012, 23, 2277-2282.

80. Adejolu, M.; Huo, L.; Rohren, E.; Santiago, L.; Yang, W.T. False-positive lesions mimicking breast cancer on FDG PET and PET/CT. AJR Am. J. Roentgenol. 2012, 198, W304-W314.

81. Koolen, B.B.; Vrancken Peeters, M.J.; Wesseling, J.; Lips, E.H.; Vogel, W.V.; Aukema, T.S.; van Werkhoven, E.; Gilhuijs, K.G.; Rodenhuis, S.; Rutgers, E.J.; et al. Association of primary tumour FDG uptake with clinical, histopathological and molecular characteristics in breast cancer patients scheduled for neoadjuvant chemotherapy. Eur. J. Nucl. Med. Mol. Imaging 2012, 39, 1830-1838.

82. Groheux, D.; Giacchetti, S.; Moretti, J.L.; Porcher, R.; Espie, M.; Lehmann-Che, J.; de Roquancourt, A.; Hamy, A.S.; Cuvier, C.; Vercellino, L.; et al. Correlation of high 18F-FDG uptake to clinical, pathological and biological prognostic factors in breast cancer. Eur. J. Nucl. Med. Mol. Imaging 2011, 38, 426-435.

83. Lyman, G.H.; Giuliano, A.E.; Somerfield, M.R.; Benson, A.B., 3rd; Bodurka, D.C.; Burstein, H.J.; Cochran, A.J.; Cody, H.S., 3rd; Edge, S.B.; Galper, S.; et al. American Society of Clinical Oncology guideline recommendations for sentinel lymph node biopsy in early-stage breast cancer. J. Clin. Oncol. 2005, 23, 7703-7720.

84. Cooper, K.L.; Meng, Y.; Harnan, S.; Ward, S.E.; Fitzgerald, P.; Papaioannou, D.; Wyld, L.; Ingram, C.; Wilkinson, I.D.; Lorenz, E. Positron emission tomography (PET) and magnetic resonance imaging (MRI) for the assessment of axillary lymph node metastases in early breast cancer: Systematic review and economic evaluation. Health Technol. Assess. 2011, 15, iii-iv, 1-134. 
85. Koolen, B.B.; Valdes Olmos, R.A.; Elkhuizen, P.H.; Vogel, W.V.; Vrancken Peeters, M.J.; Rodenhuis, S.; Rutgers, E.J. Locoregional lymph node involvement on 18F-FDG PET/CT in breast cancer patients scheduled for neoadjuvant chemotherapy. Breast Cancer Res. Treat. 2012, 135, 231-240.

86. Koolen, B.B.; Vogel, W.V.; Vrancken Peeters, M.J.; Loo, C.E.; Rutgers, E.J.; Valdes Olmos, R.A. Molecular Imaging in Breast Cancer: From Whole-Body PET/CT to Dedicated Breast PET. J. Oncol. 2012, 2012, 438647.

87. Peare, R.; Staff, R.T.; Heys, S.D. The use of FDG-PET in assessing axillary lymph node status in breast cancer: A systematic review and meta-analysis of the literature. Breast Cancer Res. Treat. 2010, 123, 281-290.

88. Riegger, C.; Herrmann, J.; Nagarajah, J.; Hecktor, J.; Kuemmel, S.; Otterbach, F.; Hahn, S.; Bockisch, A.; Lauenstein, T.; Antoch, G.; et al. Whole-body FDG PET/CT is more accurate than conventional imaging for staging primary breast cancer patients. Eur. J. Nucl. Med. Mol. Imaging 2012, 39, 852-863.

89. Piccardo, A.; Altrinetti, V.; Bacigalupo, L.; Puntoni, M.; Biscaldi, E.; Gozza, A.; Cabria, M.; Iacozzi, M.; Pasa, A.; Morbelli, S.; et al. Detection of metastatic bone lesions in breast cancer patients: Fused (18)F-Fluoride-PET/MDCT has higher accuracy than MDCT. Preliminary experience. Eur. J. Radiol. 2012, 81, 2632-2638.

90. Hahn, S.; Heusner, T.; Kummel, S.; Koninger, A.; Nagarajah, J.; Muller, S.; Boy, C.; Forsting, M.; Bockisch, A.; Antoch, G.; et al. Comparison of FDG-PET/CT and bone scintigraphy for detection of bone metastases in breast cancer. Acta Radiol. 2011, 52, 1009-1014.

91. Berland, L.L.; Silverman, S.G.; Gore, R.M.; Mayo-Smith, W.W.; Megibow, A.J.; Yee, J.; Brink, J.A.; Baker, M.E.; Federle, M.P.; Foley, W.D.; et al. Managing incidental findings on abdominal CT: White paper of the ACR incidental findings committee. J. Am. Coll. Radiol. 2010, 7, 754-773.

92. Tatsumi, M.; Cohade, C.; Mourtzikos, K.A.; Fishman, E.K.; Wahl, R.L. Initial experience with FDG-PET/CT in the evaluation of breast cancer. Eur. J. Nucl. Med. Mol. Imaging 2006, 33, 254-262.

93. Havrilesky, L.J.; Kulasingam, S.L.; Matchar, D.B.; Myers, E.R. FDG-PET for management of cervical and ovarian cancer. Gynecol. Oncol. 2005, 97, 183-191.

94. Zimny, M.; Siggelkow, W. Positron emission tomography scanning in gynecologic and breast cancers. Curr. Opin. Obst. Gynecol. 2003, 15, 69-75.

95. Zimny, M.; Siggelkow, W.; Schroder, W.; Nowak, B.; Biemann, S.; Rath, W.; Buell, U. 2-[Fluorine-18]-fluoro-2-deoxy-d-glucose positron emission tomography in the diagnosis of recurrent ovarian cancer. Gynecol. Oncol. 2001, 83, 310-315.

96. Menzel, C.; Dobert, N.; Hamscho, N.; Zaplatnikov, K.; Vasvatekis, S.; Matic, V.; Wördehoff, N.; Grünwald, F. The influence of CA 125 and CEA levels on the results of (18)F-deoxyglucose positron emission tomography in suspected recurrence of epithelial ovarian cancer. Strahlenther. Onkol. 2004, 180, 497-501.

97. Fulham, M.J.; Carter, J.; Baldey, A.; Hicks, R.J.; Ramshaw, J.E.; Gibson, M. The impact of PET-CT in suspected recurrent ovarian cancer: A prospective multi-centre study as part of the Australian PET Data Collection Project. Gynecol. Oncol. 2009, 112, 462-468. 
98. Rieber, A.; Nussle, K.; Stohr, I.; Grab, D.; Fenchel, S.; Kreienberg, R.; Reske, S.N.; Brambs, H.J. Preoperative diagnosis of ovarian tumors with MR imaging: Comparison with transvaginal sonography, positron emission tomography, and histologic findings. AJR Am. J. Roentgenol. 2001, 177,123-129.

99. Hynninen, J.; Auranen, A.; Carpen, O.; Dean, K.; Seppanen, M.; Kemppainen, J.; Lavonius, M.; Lisinen, I., Virtanen, J.; Grénman, S. FDG PET/CT in staging of advanced epithelial ovarian cancer: Frequency of supradiaphragmatic lymph node metastasis challenges the traditional pattern of disease spread. Gynecol. Oncol. 2012, 126, 64-68.

100. Bats, A.S.; Hugonnet, F.; Huchon, C.; Bensaid, C.; Pierquet-Ghazzar, N.; Faraggi, M.; Lécuru, L. Prognostic significance of mediastinal 18F-FDG uptake in PET/CT in advanced ovarian cancer. Eur. J. Nucl. Med. Mol. Imaging 2012, 39, 474-480.

101. Yoshida, Y.; Kurokawa, T.; Kawahara, K.; Tsuchida, T.; Okazawa, H.; Fujibayashi, Y.; Yonekura, Y.; Kotsuji, F. Incremental benefits of FDG positron emission tomography over CT alone for the preoperative staging of ovarian cancer. AJR Am. J. Roentgenol. 2004, 182, 227-233.

102. Castellucci, P.; Perrone, A.M.; Picchio, M.; Ghi, T.; Farsad, M.; Nanni, C.; Messa, C.; Meriggiola, M.C.; Pelusi, G.; Al-Nahhas, A.; et al. Diagnostic accuracy of 18F-FDG PET/CT in characterizing ovarian lesions and staging ovarian cancer: Correlation with transvaginal ultrasonography, computed tomography, and histology. Nucl. Med. Commun. 2007, 28, 589-595.

103. De Iaco, P.; Musto, A.; Orazi, L.; Zamagni, C.; Rosati, M.; Allegri, V.; Cacciari, N.; Al-Nahhas, A.; Rubello, D.; Venturoli, S.; et al. FDG-PET/CT in advanced ovarian cancer staging: Value and pitfalls in detecting lesions in different abdominal and pelvic quadrants compared with laparoscopy. Eur. J. Radiol. 2011, 80, e98-e103.

104. Turlakow, A.; Yeung, H.W.; Salmon, A.S.; Macapinlac, H.A.; Larson, S.M. Peritoneal carcinomatosis: Role of (18)F-FDG PET. J. Nucl. Med. 2003, 44, 1407-1412.

105. Kim, H.W.; Won, K.S.; Zeon, S.K.; Ahn, B.C.; Gayed, I.W. Peritoneal carcinomatosis in patients with ovarian cancer: Enhanced CT versus 18F-FDG PET/CT. Clin. Nucl. Med. 2013, 38, 93-97.

106. Suzuki, A.; Kawano, T.; Takahashi, N.; Lee, J.; Nakagami, Y.; Miyagi, E.; Hirahara, F.; Togo, S.; Shimada, H.; Inoue, T. Value of $18 \mathrm{~F}-F D G$ PET in the detection of peritoneal carcinomatosis. Eur. J. Nucl. Med. Mol. Imaging 2004, 31, 1413-1420.

107. Sanli, Y.; Turkmen, C.; Bakir, B.; Iyibozkurt, C.; Ozel, S.; Has, D.; Yilmaz, E.; Topuz, S.; Yavuz, E.; Unal, S.N.; et al. Diagnostic value of PET/CT is similar to that of conventional MRI and even better for detecting small peritoneal implants in patients with recurrent ovarian cancer. Nucl. Med. Commun. 2012, 33, 509-515.

108. Drieskens, O.; Stroobants, S.; Gysen, M.; Vandenbosch, G.; Mortelmans, L.; Vergote, I. Positron emission tomography with FDG in the detection of peritoneal and retroperitoneal metastases of ovarian cancer. Gynecol. Obst. Investig. 2003, 55, 130-134.

109. Ebina, Y.; Watari, H.; Kaneuchi, M.; Takeda, M.; Hosaka, M.; Kudo, M.; Yamada, H.; Sakuragi, N. Impact of FDG PET in optimizing patient selection for cytoreductive surgery in recurrent ovarian cancer. Eur. J. Nucl. Med. Mol. Imaging 2014, 41, 446-451. 
110. Fruscio, R.; Sina, F.; Dolci, C.; Signorelli, M.; Crivellaro, C.; Dell’Anna, T.; Cuzzocrea, M.; Guerra, L.; Milani, R.; Messa, C. Preoperative 18F-FDG PET/CT in the management of advanced epithelial ovarian cancer. Gynecol. Oncol. 2013, 131, 689-693.

111. Risum, S.; Loft, A.; Engelholm, S.A.; Hogdall, E.; Berthelsen, A.K.; Nedergaard, L.; Lundvall, L.; Høgdall, C. Positron emission tomography/computed tomography predictors of overall survival in stage IIIC/IV ovarian cancer. Int. J. Gynecol. Cancer 2012, 22, 1163-1169.

112. Chung, H.H.; Kwon, H.W.; Kang, K.W.; Park, N.H.; Song, Y.S.; Chung, J.K.; Kang, S.B.; Kim, J.W. Prognostic value of preoperative metabolic tumor volume and total lesion glycolysis in patients with epithelial ovarian cancer. Ann. Surg. Oncol. 2012, 19, 1966-1972.

113. Patel, C.N.; Nazir, S.A.; Khan, Z.; Gleeson, F.V.; Bradley, K.M. 18F-FDG PET/CT of cervical carcinoma. AJR Am. J. Roentgenol. 2011, 196, 1225-1233.

114. Chung, H.H.; Cheon, G.J.; Kang, K.W.; Kim, J.W.; Park, N.H.; Song, Y.S. Preoperative PET/CT FDG standardized uptake value of pelvic lymph nodes as a significant prognostic factor in patients with uterine cervical cancer. Eur. J. Nucl. Med. Mol. Imaging 2014, 41, 674-681.

115. Kidd, E.A.; Siegel, B.A.; Dehdashti, F.; Grigsby, P.W. Pelvic lymph node F-18 fluorodeoxyglucose uptake as a prognostic biomarker in newly diagnosed patients with locally advanced cervical cancer. Cancer 2010, 116, 1469-1475.

116. Wright, J.D.; Dehdashti, F.; Herzog, T.J.; Mutch, D.G.; Huettner, P.C.; Rader, J.S.; Gibb, R.K.; Powell, M.A.; Gao, F.; Siegel, B.A.; et al. Preoperative lymph node staging of early-stage cervical carcinoma by [18F]-fluoro-2-deoxy-d-glucose-positron emission tomography. Cancer 2005, 104, 2484-2491.

117. Sironi, S.; Buda, A.; Picchio, M.; Perego, P.; Moreni, R.; Pellegrino, A.; Colombo, M.; Mangioni, C.; Messa, C.; Fazio, F. Lymph node metastasis in patients with clinical early-stage cervical cancer: Detection with integrated FDG PET/CT. Radiology 2006, 238, 272-279.

118. Herrera, F.G.; Prior, J.O. The role of PET/CT in cervical cancer. Front. Oncol. 2013, 3, 34.

119. Gouy, S.; Morice, P.; Narducci, F.; Uzan, C.; Gilmore, J.; Kolesnikov-Gauthier, H.; Querleu, D.; Haie-Meder, C.; Leblanc, E. Nodal-staging surgery for locally advanced cervical cancer in the era of PET. Lancet Oncol. 2012, 13, e212-e220.

120. Choi, H.J.; Roh, J.W.; Seo, S.S.; Lee, S.; Kim, J.Y.; Kim, S.K.; Kang, K.W.; Lee, J.S.; Jeong, J.Y.; Park, S.Y. Comparison of the accuracy of magnetic resonance imaging and positron emission tomography/computed tomography in the presurgical detection of lymph node metastases in patients with uterine cervical carcinoma: A prospective study. Cancer 2006, 106, 914-922.

121. Kitajima, K.; Yamasaki, E.; Kaji, Y.; Murakami, K.; Sugimura, K. Comparison of DWI and PET/CT in evaluation of lymph node metastasis in uterine cancer. World J. Radiol. 2012, 4, $207-214$.

122. Im, H.J.; Yoon, H.J.; Lee, E.S.; Kim, T.S.; Kim, J.Y.; Chung, J.K.; Park, S.Y. Prognostic implication of retrocrural lymph node involvement revealed by (18)F-FDG PET/CT in patients with uterine cervical cancer. Nucl. Med. Commun. 2014, 35, 268-275.

123. Zhao, Q.; Feng, Y.; Mao, X.; Qie, M. Prognostic value of fluorine-18-fluorodeoxyglucose positron emission tomography or PET-computed tomography in cervical cancer: A meta-analysis. Int. J. Gynecol. Cancer 2013, 23, 1184-1190. 
124. Gouy, S.; Morice, P.; Narducci, F.; Uzan, C.; Martinez, A.; Rey, A.; Bentivegna, E.; Pautier, P.; Deandreis, D.; Querleu, D.; et al. Prospective multicenter study evaluating the survival of patients with locally advanced cervical cancer undergoing laparoscopic para-aortic lymphadenectomy before chemoradiotherapy in the era of positron emission tomography imaging. J. Clin. Oncol. 2013, 31, 3026-3033.

125. Margulies, A.L.; Peres, A.; Barranger, E.; Perreti, I.; Brouland, J.F.; Toubet, E.; Sarda-Mantel, L.E.; Thoury, A.; Chis, C.; Walker, F.; et al. Selection of patients with advanced-stage cervical cancer for para-aortic lymphadenectomy in the era of PET/CT. Anticancer Res. 2013, 33, 283-286.

126. Liu, F.Y.; Yen, T.C.; Chen, M.Y.; Lai, C.H.; Chang, T.C.; Chou, H.H.; , Hong, J.H.; Chen, Y.R.; $\mathrm{Ng}, \mathrm{K} . \mathrm{K}$. Detection of hematogenous bone metastasis in cervical cancer: 18F-fluorodeoxyglucosepositron emission tomography versus computed tomography and magnetic resonance imaging. Cancer 2009, 115, 5470-5480.

127. Akkas, B.E.; Demirel, B.B.; Vural, G.U. Clinical impact of ${ }^{18}$ F-FDG PET/CT in the pretreatment evaluation of patients with locally advanced cervical carcinoma. Nucl. Med. Commun. 2012, 33, 1081-1088.

128. Keu, K.V.; Iagaru, A.H. The clinical use of PET/CT in the evaluation of melanoma. Methods Mol. Biol. 2014, 1102, 553-580.

129. Danielsen, M.; Hojgaard, L.; Kjaer, A.; Fischer, B.M. Positron emission tomography in the follow-up of cutaneous malignant melanoma patients: A systematic review. Am. J. Nucl. Med. Mol. Imaging 2013, 4, 17-28.

130. Eigtved, A.; Andersson, A.P.; Dahlstrom, K.; Rabol, A.; Jensen, M.; Holm, S.; Sørensen, S.S.; Drzewiecki, K.T.; Højgaard, L.; Friberg, L. Use of fluorine-18 fluorodeoxyglucose positron emission tomography in the detection of silent metastases from malignant melanoma. Eur. J. Nucl. Med. 2000, 27, 70-75.

131. Rinne, D.; Baum, R.P.; Hor, G.; Kaufmann, R. Primary staging and follow-up of high risk melanoma patients with whole-body $18 \mathrm{~F}$-fluorodeoxyglucose positron emission tomography: Results of a prospective study of 100 patients. Cancer 1998, 82, 1664-1671.

132. Holder, W.D., Jr.; White, R.L., Jr.; Zuger, J.H.; Easton, E.J., Jr.; Greene, F.L. Effectiveness of positron emission tomography for the detection of melanoma metastases. Ann. Surg. 1998, 227, 764-771.

133. Kwee, T.C.; Kwee, R.M.; Nievelstein, R.A. Imaging in staging of malignant lymphoma: A systematic review. Blood 2008, 111, 504-516.

134. Munker, R.; Glass, J.; Griffeth, L.K.; Sattar, T.; Zamani, R.; Heldmann, M.; Shi, R.; Lilien, D.L. Contribution of PET imaging to the initial staging and prognosis of patients with Hodgkin's disease. Ann. Oncol. 2004, 15, 1699-1704.

135. Rigacci, L.; Vitolo, U.; Nassi, L.; Merli, F.; Gallamini, A.; Pregno, P.; Alvarez, I.; Salvi, F.; Sancetta, R.; Castagnoli, A.; et al. Positron emission tomography in the staging of patients with Hodgkin's lymphoma. A prospective multicentric study by the Intergruppo Italiano Linfomi. Ann. Hematol. 2007, 86, 897-903.

136. Hutchings, M.; Loft, A.; Hansen, M.; Pedersen, L.M.; Berthelsen, A.K.; Keiding, S.; D'Amore, F.; Boesen, A.M.; Roemer, L.; Specht, L. Position emission tomography with or without computed tomography in the primary staging of Hodgkin's lymphoma. Haematologica 2006, 91, 482-489. 
137. Cheson, B.D. Role of functional imaging in the management of lymphoma. J. Clin. Oncol. 2011, 29, 1844-1854.

138. Rodriguez-Vigil, B.; Gomez-Leon, N.; Pinilla, I.; Hernandez-Maraver, D.; Coya, J.; Martin-Curto, L.; Madero, R. PET/CT in lymphoma: Prospective study of enhanced full-dose PET/CT versus unenhanced low-dose PET/CT. J. Nucl. Med. 2006, 47, 1643-1648.

139. Chalaye, J.; Luciani, A.; Enache, C.; Beaussart, P.; Lhermite, C.; Evangelista, E.; Sasanelli, M.; Safar, V.; Meignan, M.; Haioun, C.; et al. Clinical impact of contrast-enhanced CT combined with low-dose F-FDG PET/CT on routine lymphoma patient management. Leuk. Lymphoma 2014, doi:10.3109/10428194.2014.900761.

140. Pinilla, I.; Gomez-Leon, N.; del Campo-Del Val, L.; Hernandez-Maraver, D.; Rodriguez-Vigil, B.; Jover-Diaz, R.; Coya, J. Diagnostic value of CT, PET and combined PET/CT performed with low-dose unenhanced CT and full-dose enhanced CT in the initial staging of lymphoma. Q. J. Nucl. Med. Mol. Imaging 2011, 55, 567-575.

141. Elstrom, R.L.; Leonard, J.P.; Coleman, M.; Brown, R.K. Combined PET and low-dose, noncontrast $\mathrm{CT}$ scanning obviates the need for additional diagnostic contrast-enhanced $\mathrm{CT}$ scans in patients undergoing staging or restaging for lymphoma. Ann. Oncol. 2008, 19, 1770-1773.

142. El-Galaly, T.C.; d'Amore, F.; Mylam, K.J.; de Nully Brown, P.; Bogsted, M.; Bukh, A.; Specht, L.; Loft, A.; Iyer, V.; Hjorthaug, K.; et al. Routine bone marrow biopsy has little or no therapeutic consequence for positron emission tomography/computed tomography-staged treatment-naive patients with Hodgkin lymphoma. J. Clin. Oncol. 2012, 30, 4508-4514.

143. Khan, A.B.; Barrington, S.F.; Mikhaeel, N.G.; Hunt, A.A.; Cameron, L.; Morris, T.; Carr, R. PET-CT staging of DLBCL accurately identifies and provides new insight into the clinical significance of bone marrow involvement. Blood 2013, 122, 61-67.

144. Avigdor, A. Staging DLBCL: Bone marrow biopsy or PET-CT? Blood 2013, 122, 4-5.

145. Le Dortz, L.; de Guibert, S.; Bayat, S.; Devillers, A.; Houot, R.; Rolland, Y.; Cuggia, M.; Le Jeune, F.; Bahri, H.; Barge, M.L.; et al. Diagnostic and prognostic impact of 18F-FDG PET/CT in follicular lymphoma. Eur. J. Nucl. Med. Mol. Imaging 2010, 37, 2307-2314.

146. Luminari, S.; Biasoli, I.; Arcaini, L.; Versari, A.; Rusconi, C.; Merli, F.; Spina, M.; Ferreri, A.J.; Zinzani, P.L.; Gallamini, A.; et al. The use of FDG-PET in the initial staging of 142 patients with follicular lymphoma: A retrospective study from the FOLL05 randomized trial of the Fondazione Italiana Linfomi. Ann. Oncol. 2013, 24, 2108-2112.

147. Bodet-Milin, C.; Touzeau, C.; Leux, C.; Sahin, M.; Moreau, A.; Maisonneuve, H.; Morineau, N.; Jardel, H.; Moreau, P.; Gallazini-Crépin, C.; et al. Prognostic impact of 18F-fluoro-deoxyglucose positron emission tomography in untreated mantle cell lymphoma: A retrospective study from the GOELAMS group. Eur. J. Nucl. Med. Mol. Imaging 2010, 37, 1633-1642.

148. Karam, M.; Ata, A.; Irish, K.; Feustel, P.J.; Mottaghy, F.M.; Stroobants, S.G.; Verhoef, G.E.; Chundru, S.; Douglas-Nikitin, V.; Oliver Wong, C.Y.; et al. FDG positron emission tomography/computed tomography scan may identify mantle cell lymphoma patients with unusually favorable outcome. Nucl. Med. Commun. 2009, 30, 770-778.

149. Barrington, S.F.; Mikhaeel, N.G.; Kostakoglu, L.; Meignan, M.; Hutchings, M.; MÜeller, S.P.; Lawrence, H.S.; Zucca, E.; Fisher, R.I.; Trotman, J.; et al. Role of Imaging in the Staging and 
Response Assessment of Lymphoma: Consensus of the International Conference on Malignant Lymphomas Imaging Working Group. J. Clin. Oncol. 2014, doi:10.1200/JCO.2013.54.8800.

150. Sim, Y.T.; Poon, F.W. Imaging of solitary pulmonary nodule-a clinical review. Quant. Imaging Med. Surg. 2013, 3, 316-326.

151. Erasmus, J.J.; McAdams, H.P.; Connolly, J.E. Solitary pulmonary nodules: Part II. Evaluation of the indeterminate nodule. Radiographics 2000, 20, 59-66.

152. Gurney, J.W.; Lyddon, D.M.; McKay, J.A. Determining the likelihood of malignancy in solitary pulmonary nodules with Bayesian analysis. Part II. Application. Radiology 1993, 186, 415-422.

153. Bryant, A.S.; Cerfolio, R.J. The maximum standardized uptake values on integrated FDG-PET/CT is useful in differentiating benign from malignant pulmonary nodules. Ann. Thoracic Surg. 2006, $82,1016-1020$.

154. Lowe, V.J.; Fletcher, J.W.; Gobar, L.; Lawson, M.; Kirchner, P.; Valk, P.; Karis, J.; Hubner, K.; Delbeke, D.; Heiberg, E.V.; et al. Prospective investigation of positron emission tomography in lung nodules. J. Clin. Oncol. 1998, 16, 1075-1084.

155. Hashimoto, Y.; Tsujikawa, T.; Kondo, C.; Maki, M.; Momose, M.; Nagai, A.; Ohnuki, T.; Nishikawa, T.; Kusakabe, K. Accuracy of PET for diagnosis of solid pulmonary lesions with 18F-FDG uptake below the standardized uptake value of 2.5. J. Nucl. Med. 2006, 47, 426-431.

156. Nguyen, N.C.; Kaushik, A.; Wolverson, M.K.; Osman, M.M. Is there a common SUV threshold in oncological FDG PET/CT, at least for some common indications? A retrospective study. Acta Oncol. 2011, 50, 670-677.

157. Kim, S.K.; Allen-Auerbach, M.; Goldin, J.; Fueger, B.J.; Dahlbom, M.; Brown, M.; Czernin, J.; Schiepers, C. Accuracy of PET/CT in characterization of solitary pulmonary lesions. J. Nucl. Med. 2007, 48, 214-220.

158. Deppen, S.; Putnam, J.B., Jr.; Andrade, G.; Speroff, T.; Nesbitt, J.C.; Lambright, E.S.; Massion, P.P.; Walker, R.; Grogan, E.L. Accuracy of FDG-PET to diagnose lung cancer in a region of endemic granulomatous disease. Ann. Thoracic Surg. 2011, 92, 428-433.

159. Gould, M.K.; Maclean, C.C.; Kuschner, W.G.; Rydzak, C.E.; Owens, D.K. Accuracy of positron emission tomography for diagnosis of pulmonary nodules and mass lesions: A meta-analysis. JAMA 2001, 285, 914-924.

160. Orlacchio, A.; Schillaci, O.; Antonelli, L.; D’Urso, S.; Sergiacomi, G.; Nicoli, P.; Simonetti, G. Solitary pulmonary nodules: Morphological and metabolic characterisation by FDG-PET-MDCT. La Radiol. Med. 2007, 112, 157-173.

161. Zhang, L.; Wang, Y.; Lei, J.; Tian, J.; Zhai, Y. Dual time point 18FDG-PET/CT versus single time point $18 \mathrm{FDG}-\mathrm{PET} / \mathrm{CT}$ for the differential diagnosis of pulmonary nodules: A meta-analysis. Acta Radiol. 2013, 54, 770-777.

162. Gould, M.K.; Fletcher, J.; Iannettoni, M.D.; Lynch, W.R.; Midthun, D.E.; Naidich, D.P.; Ost, D.E.; American College of Chest Physicians. Evaluation of patients with pulmonary nodules: When is it lung cancer? ACCP evidence-based clinical practice guidelines (2nd edition). Chest 2007, 132, 108S-130S.

163. Wahl, R.L.; Jacene, H.; Kasamon, Y.; Lodge, M.A. From RECIST to PERCIST: Evolving considerations for PET Response Criteria in Solid Tumors. J. Nucl. Med. 2009, 50, 122S-150S.

164. Weber, W.A. Assessing Tumor Response to Therapy. J. Nucl. Med. 2009, 50, 1S-10S 
165. Minn, H.; Paul, R.; Ahonen, A. Evaluation of treatment response to radiotherapy in head and neck cancer with fluorine-18 fluorodeoxyglucose. J. Nucl. Med. 1988, 29, 1521-1525.

166. Wahl, R.L.; Zasadny, K.; Helvie, M.; Hutchins, G.D.; Weber, B.; Cody, R. Metabolic monitoring of breast cancer chemo-hormonotherapy using positron emission tomography: Initial evaluation. J. Clin. Oncol. 1993, 11, 2101-2111.

167. Hoekstra, O.S.; van Lingen, A.; Ossenkoppele, G.J.; Golding, R.; Teule, G.J. Early response monitoring in malignant lymphoma using fluorine-18 fluorodeoxyglucose single-photon emission tomography. Eur. J. Nucl. Med. 1993, 20, 1214-1217.

168. Mikhaeel, N.G.; Hutchings, M.; Fields, P.A.; O’Doherty, M.J.; Timothy, A.R. FDG-PET after two to three cycles of chemotherapy predicts progression-free and overall survival in high-grade non-Hodgkin lymphoma. Ann. Oncol. 2005, 16, 1514-1523.

169. Hutchings, M.; Loft, A.; Hansen, M.; Pedersen, L.M.; Buhl, T.; Jurlander, J.; Buus, S.; Keiding, S.; D'Amore, F.; Boesen, A.M.; et al. FDG-PET after two cycles of chemotherapy predicts treatment failure and progression-free survival in Hodgkin lymphoma. Blood 2006, 107, 52-59.

170. Gallamini, A.; Rigacci, L.; Merli, F.; Nassi, L.; Bosi, A.; Capodanno, I.; Vitolo, U.; Sancetta, R.; Iannitto, E.; Trentin, L.; et al. The predictive value of positron emission tomography scanning performed after two courses of standard therapy on treatment outcome in advanced stage Hodgkin's disease. Haematologica 2006, 91, 475-481.

171. Cerci, J.J.; Pracchia, L.F.; Linardi, C.C.; Delbeke, D.; Izaki, M.; Trindade, E.; Soares, J., Jr; Buccheri. V.; Meneghetti, J.C. 18F-FDG PET after 2 cycles of ABVD predicts event-free survival in early and advanced Hodgkin lymphoma. J. Nucl. Med. 2010, 51, 1337-1343.

172. Casasnovas, R.O.; Meignan, M.; Berriolo-Riedinger, A.; Bardet, S.; Julian, A.; Thieblemont, C.; Vera, P.; Bologna, S.; Brière, J.; Jais, J.P.; et al. SUVmax reduction improves early prognosis value of interim positron emission tomography scans in diffuse large B-cell lymphoma. Blood 2011, 118, 37-43.

173. Terasawa, T.; Lau, J.; Bardet, S.; Couturier, O.; Hotta, T.; Hutchings, M.; Nihashi, T.; Nagai, H. Fluorine-18-fluorodeoxyglucose positron emission tomography for interim response assessment of advanced stage Hodgkin's lymphoma and diffuse large B-cell lymphoma: A systematic review. J. Clin. Oncol. 2009, 27, 1906-1914.

174. Meignan, M.; Gallamini, A.; Haioun, C.; Polliack, A. Report on the Second International Workshop on interim positron emission tomography in lymphoma held in Menton, France, 8-9 April 2010. Leuk. Lymphoma 2010, 51, 217111-217180.

175. Meignan, M.; Itti, E.; Gallamini, A.; Hioun, C. Interim 18F-fluorodeoxyglucose positron emission tomography in diffuse large B-cell lymphoma: Qualitative or quantitative InterpretationWhere do we stand? Leuk. Lymphoma 2009, 50, 1753-1756.

176. Gallamini, A.; Fiore, F.; Sorasio, R.; Meignan, M. Interim positron emission tomography scan in Hodgkin lymphoma: Definitions, interpretation rules, and clinical validation. Leuk. Lymphoma 2009, 50, 1761-1764.

177. Gallamini, A. Positron emission tomography scanning: A new paradigm for the management of Hodgkin's lymphoma. Haematologica 2010, 95, 1046-1048.

178. Facey, K.; Bradbury, I.; Laking, G.; Payne, E. Overview of the clinical effectiveness of positron emission tomography imaging in selected cancers. Health Technol. Assess. 2007, 11, 44. 
179. Fletcher, J.W.; Djulbegovic, B.; Soares, H.P.; Siegel, B.A.; Lowe, V.J.; Lyman, G.H.; Coleman, R.E.; Wahl, R.; Paschold, J.C.; Avril, N.; et al. Recommendations on the use of 18 F-FDG PET in oncology. J. Nucl. Med. 2008, 49, 480-508.

180. Shankar, L.K.; Hoffman, J.M.; Bacharach, S.; Graham, M.M.; Karp, J.; Lammertsma, A.A.; Larson, S.; Mankoff, D.A.; Siegel, B.A.; van den Abbeele, A.; et al. Consensus recommendations for the use of $18 \mathrm{~F}-\mathrm{FDG}$ PET as an indicator of therapeutic response in patients in National Cancer Institute trials. J. Nucl. Med. 2006, 47, 1059-1066.

181. Young, H.; Baum, R.; Cremerius, U.; Herholz, K.; Hoekstra, O.; Lammertsma, A.A.; Pruim, J.; Price, P. Measurement of clinical and subclinical tumour response using [18F]-fluorodeoxyglucose and positron emission tomography: Review and 1999 EORTC recommendations. European Organization for Research and Treatment of Cancer (EORTC) PET Study Group. Eur. J. Cancer 1999, 35, 1773-1782.

182. Contractor, K.B.; Aboagye, E.O. Monitoring predominantly cytostatic treatment response with 18F-FDG PET. J. Nucl. Med. 2009, 50, 97S-105S.

183. Castell, F.; Cook, G.J. Quantitative techniques in 18 FDG PET scanning in oncology. Br. J. Cancer 2008, 98, 1597-1601.

184. Keyes, J.W., Jr. SUV: Standard uptake or silly useless value? J. Nucl. Med. 1995, 36, 1836-1839.

185. Kostakoglu, L.; Goldsmith, S.J.; Leonard, J.P.; Christos, P.; Furman, R.R.; Atasever, T.; Chandramouly, A.; Verma, S.; Kothari, P.; Coleman, M. FDG-PET after 1 cycle of therapy predicts outcome in diffuse large cell lymphoma and classic Hodgkin disease. Cancer 2006, 107, 2678-2687.

186. Zinzani, P.L.; Rigacci, L.; Stefoni, V.; Broccoli, A.; Puccini, B.; Castagnoli, A.; Vaggelli, L.; Zanoni, L.; Argnani, L.; Baccarani, M.; et al. Early interim 18 F-FDG PET in Hodgkin's lymphoma: Evaluation on 304 patients. Eur. J. Nucl. Med. Mol. Imaging 2012, 39, 4-12.

187. Hutchings, M.; Mikhaeel, N.G.; Fields, P.A.; Nunan, T.; Timothy, A.R. Prognostic value of interim FDG-PET after two or three cycles of chemotherapy in Hodgkin lymphoma. Ann. Oncol. 2005, 16, 1160-1168.

188. Gallamini, A.; Hutchings, M.; Rigacci, L.; Specht, L.; Merli, F.; Hansen, M.; Patti, C.; Loft, A.; di Raimondo, F.; D’Amore, F.; et al. Early interim 2-[18F]fluoro-2-deoxy-D glucose positron emission tomography is prognostically superior to international prognostic score in advancedstage Hodgkin's lymphoma: A report from a joint Italian-Danish study. J. Clin. Oncol. 2007, 25, 3746-3752.

189. Lin, C.; Itti, E.; Haioun, C.; Petegnief, Y.; Luciani, A.; Dupuis, J.; Talbot, J.N.; Rahmouni, A.; Meignan, M. Early 18F-FDG PET for prediction of prognosis in patients with diffuse large B-Cell lymphoma: SUV-based assessment versus visual analysis. J. Nucl. Med. 2007, 48, 1626-1632.

190. Moskowitz, C.H.; Schoder, H.; Teruya-Feldstein, J.; Sima, C.; Iasonos, A.; Portlock, C.S.; Straus, D.; Noy, A.; Palomba, M.L.; O’Connor, O.A.; et al. Risk adapted dose-dense immunochemoterapy determined by interim FDG-PET in advanced stage diffuse large B-cell lymphoma. J. Clin. Oncol. 2010, 28, 1896-1903.

191. Pregno, P.; Chiappella, A.; Bello, M.; Botto, B.; Ferrero, S.; Franceschetti, S.; Giunta, F.; Ladetto, M.; Limerutti, G.; Menga, M.; et al. Interim 18-FDG-PET/CT failed to predict the 
outcome in diffuse large B-cell lymphoma patients treated at the diagnosis with rituximabCHOP. Blood 2012, 119, 2066-2073.

192. Cashen, A.F.; Dehdashti, F.; Luo, J.; Homb, A.; Siegel, B.A.; Bartlett, N.L. 18-FDG-PET/CT for early response assessment in diffuse large B-cell lymphoma: Poor predictive value of international harmonization project interpretation. J. Nucl. Med. 2011, 52, 386-392.

193. Gallamini, A.; Kostakoglu, L. Interim FDG-PET in Hodgkin lymphoma: A compass for a safe navigation in clinical trials? Blood 2012, 120, 4913-4920.

194. Dupuis, J.; Berriolo-Riedinger, A.; Julian, A.; Brice, P.; Tychyj-Pinel, C.; Tilly, H.; Mounier, N.; Gallamini, A.; Feugier, P.; Soubeyran, P.; et al. Impact of [(18)F]fluorodeoxyglucose positron emission tomography response evaluation in patients with high tumor burden follicular lymphoma treated with immunochemotherapy: A prospective study from the Groupe d'Etudes des Lymphomes de l'Adulte and GOELAMS. J. Clin. Oncol. 2012, 30, 4317-4322.

195. Pellegrini, C.; Casadei, B.; Derenzini, E.; Stefoni, V.; Derenzini, E.; Gandolfi, L.; Casadei, B.; Maglie, R.; Pileri, S.; Zinzani, P.L. Prognostic Value of Interim Positron Emission Tomography in Patients With Peripheral T-Cell Lymphoma. Oncologist 2014, 19, 746-750.

196. Meignan, M.; Gallamini, A.; Haioun, C. Report on the first international workshop on interim-PET scan in Lymphoma. Leuk. Lymphoma 2009, 50, 1257-1260.

197. Biggi, A.; Gallamini, A.; Chauvie, S.; Hutchings, M.; Kostakoglu, L.; Gregianin, M.; Meignan, M.; Malkowski, B.; Hofman, M.S. Barrington, S.F.; et al. International validation study for interim PET in ABVD-treated, advanced-stage Hodgkin lymphoma: Interpretation criteria and concordance rate among reviewers. $J$. Nucl. Med. 2013, 54, 683-690.

198. Gallamini, A.; Barrington, S.F.; Biggi, A.; Chauvie, S.; Kostakoglu, L.; Gregianin, M.; Meignan, M.; Mikhaeel, G.N.; Loft, A.; Zaucha, J.M.; et al. The predictive role of interim Positron Emission Tomography on Hodgkin lymphoma treatment outcome is confirmed using the 5-point scale interpretation criteria. Haematologica 2014, 99, 1107-1113.

199. Schwarz-Dose, J.; Untch, M.; Tiling, R.; Sassen, S.; Mahner, S.; Kahlert, S.; Harbeck, N.; Lebeau, A.; Brenner, W.; Schwaiger, M.; et al. Monitoring primary systemic therapy of large and locally advanced breast cancer by using sequential positron emission tomography imaging with [18F] fluorodeoxyglucose. J. Clin. Oncol. 2009, 27, 535-541.

200. Martoni, A.A.; Zamagni, C.; Quercia, S.; Rosati, M.; Cacciari, N.; Bernardi, A.; Musto, A.; Fanti, S.; Santini, D.; Taffurelli, M. Early (18)F-2-fluoro-2-deoxy-D-glucose positron emission tomography may identify a subset of patients with estrogen receptor-positive breast cancer who will not respond optimally to preoperative chemotherapy. Cancer 2010, 116, 805-813.

201. Keam, B.; Im, S.A.; Koh, Y.; Han, S.W.; Oh, D.Y.; Cho, N.; Kim, J.H.; Han, W.; Kang, K.W.; Moon, W.K.; et al. Early metabolic response using FDG PET/CT and molecular phenotypes of breast cancer treated with neoadjuvant chemotherapy. BMC Cancer 2011, 11, 452.

202. Schelling, M.; Avril, N.; Nahrig, J.; Kuhn, W.; Römer, W.; Sattler, D.; Werner, M.; Dose, J.; Jänicke, F.; Graeff, H.; et al. Positron emission tomography using [18F]fluorodeoxyglucose for monitoring primary chemotherapy in breast cancer. J. Clin. Oncol. 2000, 18, 1689-1695.

203. Smith, I.C.; Welch, A.E.; Hutcheon, A.W.; Miller, I.D.; Payne, S.; Chilcott, F.; Waikar, S; Whitaker, T.; Ah-See, A.K.; Eremin, O.; et al. Positron emission tomography using [18F]- 
fluorodeoxy-D-glucose to predict the pathologic response of breast cancer to primary chemotherapy. J. Clin. Oncol. 2000, 18, 1676-1688.

204. Rousseau, C.; Devillers, A.; Sagan, C.; Ferrer, L.; Bridji, B.; Campion, L.; Ricaud, M.; Bourbouloux, E.; Doutriaux, I.; Clouet, M.; et al. Monitoring of early response to neoadjuvant chemotherapy in stage II and III breast cancer by [18F]fluorodeoxyglucose positron emission tomography. J. Clin. Oncol. 2006, 24, 5366-5372.

205. Hendlisz, A.; Golfinopoulos, V.; Deleporte, A.; Paesmans, M.; El Mansy, H.; Garcia, C.; Peeters, M.; Annemans, L.; Vandeputte, C.; Maetens, M.; et al. Preoperative chemosensitivity testing as predictor of treatment benefit in adjuvant stage III colon cancer (PePiTA): Protocol of a prospective BGDO (Belgian Group for Digestive Oncology) multicentric study. BMC Cancer 2013, 13, 190-199.

206. De Geus-Oei, L.F.; van Laarhoven, H.W.; Visser, E.P.; Hermsen, R.; van Hoorn, B.A.; Kamm, Y.J.; Krabbe, P.F.; Corstens, F.H.; Punt, C.J.; Oyen, W.J. Chemotherapy response evaluation with FDG-PET in patients with colorectal cancer. Ann. Oncol. 2008, 19, 348-352.

207. Byström, P.; Berglund, A.; Garske, U.; Jacobsson, H.; Sundin, A.; Nygren, P.; Frödin, J.E.; Glimelius, B. Early prediction of response to first-line chemotherapy by sequential [18 F]-2fluoro-2-deoxy-D-glucose positron emission tomography in patients with advanced colorectal cancer. Ann. Oncol. 2009, 20, 1057-1061.

208. Kim, J.; Choi, S.; Yi, H.; Lim, J.; Lee, M.; Hyun, I.; Kim, C. Prediction of response to chemotherapy using sequential F-18-fluorodeoxyglucose (FDG) positron emission tomography (PET) in patients with metastatic colorectal cancer. J. Clin. Oncol. 2007, 25, 2536.

209. Hendlisz, A.; Golfinopoulos, V.; Garcia, C.; Covas, A.; Emonts, P.; Ameye, L.; Paesmans, M.; Deleporte, A.; Machiels, G.; Toussaint, E.; et al. Serial FDG-PET/CT for early outcome prediction in patients with metastatic colorectal cancer undergoing chemotherapy. Ann. Oncol. 2012, 23, 1687-1693.

210. Yoon, D.H.; Cho, Y.; Kim, S.Y.; Nam, S.Y.; Choi, S.H.; Roh, J.L.; Lee, S.W.; Song, S.Y.; Lee, J.H.; Kim, J.S.; et al. Usefulness of interim FDG-PET after induction chemotherapy in patients with locally advanced squamous cell carcinoma of the head and neck receiving sequential induction chemotherapy followed by concurrent chemoradiotherapy. Int. J. Radiat. Oncol. Biol. Phys. 2011, 81, 118-125.

211. Ceulemans, G.; Voordeckers, M.; Farrag, A.; Verdriers, D.; Storme, G.; Everaert, H. Can 18-FDG-PET during radiotherapy replace post-therapy scanning for detection/demonstration of tumor response in head and neck cancer? Int. J. Radiat. Oncol. Biol. Phys. 2011, 81, 938-942.

212. Goldstraw, P.; Crowley, J.; Chansky, K.; Giroux, D.J.; Groome, P.A.; Rami-Porta, R.; Postmus, P.E.; Rusch, V.; Sobin, L.; International Association for the Study of Lung Cancer International Staging Committee; et al. The IASLC Lung Cancer Staging Project: Proposals for the revision of the TNM stage groupings in the forthcoming (seventh) ed. of the TNM Classification of malignant tumours. J. Thorac. Oncol. 2007, 2, 706-714.

213. Aukema, T.S.; Kappers, I.; Valdés Olmos, R.A.; Codrington, H.E.; van Tinteren, H.; van Pel, R.; Klomp, H.M.; NEL Study Group. Is 18F-FDG PET/CT useful for the early prediction of histopathologic response to neoadjuvant erlotinib in patients with non-small cell lung cancer? J. Nucl. Med. 2010, 51, 1344-1348. 
214. Hoekstra, C.J.; Stroobants, S.G.; Smit, E.F.; Vansteenkiste, J.; van Tinteren, H.; Postmus, P.E.; Golding, R.P.; Biesma, B.; Schramel, F.J.; van Zandwijk, N.; et al. Prognostic relevance of response evaluation using [18F]-2-fluoro-2-deoxy-D-glucose positron emission tomography in patients with locally advanced non-small-cell lung cancer. J. Clin. Oncol. 2005, 23, 8362-8370.

215. Lee, D.H.; Kim, S.K.; Lee, H.Y.; Lee, S.Y.; Park, S.H.; Kim, H.Y.; Kang, K.W.; Han, J.Y.; Kim, H.T.; Lee, J.S. Early prediction of response to first-line therapy using integrated 18F-FDG PET/CT for patients with advanced/metastatic non-small cell lung cancer. J. Thorac. Oncol. 2009, 4, 816-821.

216. Decoster, L.; Schallier, D.; Everaert, H.; Nieboer, K.; Meysman, M.; Neyns, B.; de Mey, J.; de Grève, J. Complete metabolic tumour response, assessed by 18-fluorodeoxyglucose positron emission tomography (18FDGPET), after induction chemotherapy predicts a favourable outcome in patients with locally advanced non-small cell lung cancer (NSCLC). Lung Cancer 2008, 62, $55-61$.

217. Jemal, A.; Murray, T.; Ward, E.; Samuels, A.; Tiwari, R.C.; Ghafoor, A.; Feuer, E.J.; Thun, M.J. Cancer statistics. CA Cancer. J. Clin. 2005, 55, 10-30.

218. Miyata, M.; Yamasaki, M.; Takahashi, T.; Murakami, K.; Tanaka, K.; Yukinori, K.; Nakajima, K.; Takiguchi, S.; Morii, E.; Hatazawa, J.; et al. Determinants of response to neoadjuvant chemotherapy for esophageal cancer using 18F-fluorodeoxiglucose Positron Emission Tomography (18F-FDGPET). Ann. Surg. Oncol. 2013, doi:10.1245/s10434-013-3343-5.

219. Weber, W.A.; Ott, K.; Becker, K.; Dittler, H.J.; Helmberger, H.; Avril, N.E.; Meisetschläger, G.; Busch, R.; Siewert, J.R.; Schwaiger, M.; et al. Prediction of response to preoperative chemotherapy in adenocarcinomas of the esophagogastric junction by metabolic imaging. J. Clin. Oncol. 2001, 19, 3058-3065.

220. Lordick, F.; Ott, K.; Krause, B.J.; Weber, W.A.; Becker, K.; Stein, H.J.; Lorenzen, S.; Schuster, T.; Wieder, H.; Herrmann, K.; et al. PET to assess early metabolic response and to guide treatment of adenocarcinoma of the oesophagogastric junction: The MUNICON phase II trial. Lancet Oncol. 2007, 8, 797-805.

221. Krause, B.J.; Herrmann, K.; Wieder, H.; Meyer zum Buschenfelde, C. 18F-FDG PET and 18F-FDG PET/CT for assessing response to therapy in esophageal cancer. J. Nucl. Med. 2009, 50, 89S-96S.

222. Miller, A.B.; Hoogstraten, B.; Staquet, M.; Winkler, A. Reporting results of cancer treatment. Cancer 1981, 47, 207-214.

223. Therasse, P.; Arbuck, S.G.; Eisenhauer, E.A.; Wanders, J.; Kaplan, R.S.; Rubinstein, L.; Verweij, J.; van Glabbeke, M.; van Oosterom, A.T.; Christian, M.C.; et al. New guidelines to evaluate the response to treatment in solid tumors. European Organization for Research and Treatment of Cancer, National Cancer Institute of the United States, National Cancer Institute of Canada. J. Natl. Cancer Inst. 2000, 92, 205-216.

224. Eisenhauer, E.A.; Therasse, P.; Bogaerts, J.; Schwartz, L.H.; Sargent, D.; Ford, R.; Dancey, J.; Arbuck, S.; Gwyther, S.; Mooney, M.; et al. New response evaluation criteria in solid tumors: Revised RECIST guideline (version1.1). Eur. J. Cancer 2009, 45, 228-247.

225. Moertel, C.G.; Hanley, J.A. The effect of measuring error on the results of therapeutic trials in advanced cancer. Cancer 1976, 38, 388-394. 
226. Karrison, T.G.; Maitland, M.L.; Stadler, W.M.; Ratain, M.J. Design of phase II cancer trials using a continuous endpoint of change in tumor size: Application to a study of sorafenib and erlotinib in non small-cell lung cancer. J. Natl. Cancer Inst. 2007, 99, 1455-1461.

227. Michaelis, L.C.; Ratain, M.J. Measuring response in a post-RECIST world: From black and white to shades of grey. Nat. Rev. Cancer 2006, 6, 409-414.

228. Radford, J.A.; Cowan, R.A.; Flanagan, M.; Dunn, G.; Crowther, D.; Johnson, R.J.; Eddleston, B. The significance of residual mediastinal abnormality on the chest radiograph following treatment for Hodgkin's disease. J. Clin. Oncol. 1988, 6, 940-946.

229. Surbone, A.; Longo, D.L.; de Vita, V.T., Jr.; Ihde, D.C.; Duffey, P.L.; Jaffe, E.S.; Solomon, D.; Hubbard, S.M.; Young, R.C. Residual abdominal masses in aggressive non-Hodgkin's lymphoma after combination chemotherapy: Significance and management. J. Clin. Oncol. 1988, 6, 1832-1837.

230. Naumann, R.; Vaic, A.; Beuthien-Baumann, B.; Bredow, J.; Kropp, J.; Kittner, T.; Franke, W.G.; Ehninger, G. Prognostic value of positron emission tomography in the evaluation of post-treatment residual mass in patients with Hodgkin's disease and non-Hodgkin's lymphoma. Br. J. Haematol. 2001, 115, 793-800.

231. Canellos, G.P. Residual mass in lymphoma may not be residual disease. J. Clin. Oncol. 1988, 6, 931-933.

232. Cheson, B.D.; Horning, S.J.; Coiffier, B.; Fisher, R.I.; Connors, J.M.; Lister, T.A.; Vose, J.; Grillo-López, A.; Hagenbeek, A.; et al. Report of an international workshop to standardize response criteria for non-Hodgkin's lymphomas. NCI Sponsored International Working Group. J. Clin. Oncol. 1999, 17, 1244.

233. Porceddu, S.V.; Pryor, D.I.; Burmeister, E.; Burmeister, B.H.; Poulsen, M.G.; Foote, M.C.; Panizza, B.; Coman, S.; McFarlane, D.; Coman, W.; et al. Results of a prospective study of positron emission tomography-directed management of residual nodal abnormalities in node-positive head and neck cancer after definitive radiotherapy with or without systemic therapy. Head Neck 2011, 33, 1675-1682.

234. Van den Abbeele, A.D. The Lessons of GIST_PET and PET/CT: A New Paradigm for Imaging. Oncologist 2008, 13, 8-13.

235. Forner, A.; Ayuso, C.; Varela, M.; Rimola, J.; Hessheimer, A.J.; de Lope, C.R., Reig, M.; Bianchi, L.; Llovet, J.M.; Bruix, J. Evaluation of tumor response after locoregional therapies in hepatocellular carcinoma: Are response evaluation criteria in solid tumors reliable? Cancer 2009, $115,616-623$.

236. Llovet, J.M.; Ricci, S.; Mazzaferro, V.; Hilgard, P.; Gane, E.; Blanc, J.F.; de Oliveira, A.C.; Santoro, A.; Raoul, J.L.; Forner, A.; et al. Sorafenib in advanced hepatocellular carcinoma. N. Engl. J. Med. 2008, 359, 378-390.

237. André, N.; Fabre, A.; Colavolpe, C.; Jacob, T.; Gaudart, J.; Coze, C.; Paris, M.; Gentet, J.C.; Guedj, E.; Michel, G.; et al. FDG PET and evaluation of posttherapeutic residual tumors in pediatric oncology: Preliminary experience. J. Pediatr. Hematol. Oncol. 2008, 30, 343-346.

238. Jadvar, H.; Connolly, L.P.; Fahey, F.H.; Shulkin, B.L. PET and PET/CT in Pediatric Oncology Semin. Nucl. Med. 2007, 37, 316-331. 
239. Brucher, B.L.; Weber, W.; Bauer, M.; Fink, U.; Avril, N.; Stein, H.J.; Werner, M.; Zimmerman, F.; Siewert, J.R.; Schwaiger, M. Neoadjuvant therapy of esophageal squamous cell carcinoma: Response evaluation by positron emission tomography. Ann. Surg. 2001, 233, 300-309.

240. Vansteenkiste, J.F.; Stroobants, S.G.; de Leyn, P.R.; Dupont, P.J.; Verbeken, E.K. Potential use of FDG-PET scan after induction chemotherapy in surgically staged IIIa-N2 non-small-cell lung cancer: A prospective pilot study. The Leuven Lung Cancer Group. Ann. Oncol. 1998, 9, 1193-1198.

241. Bryant, A.S.; Cerfolio, R.J.; Klemm, K.M.; Ojha, B. Maximum standard uptake value of mediastinal lymph nodes on integrated FDG-PET-CT predicts pathology in patients with non-small cell lung cancer. Ann. Thorac. Surg. 2006, 82, 417-422.

242. Dooms, C.; Verbeken, E.; Stroobants, S.; Nackaerts, K.; de Leyn, P.; Vansteenkiste, J. Prognostic stratification of stage IIIA-N2 non-small-cell lung cancer after induction chemotherapy: A model based on the combination of morphometric pathologic response in mediastinal nodes and primary tumor response on serial 18-fluoro-2-deoxy-glucose positron emission tomography. J. Clin. Oncol. 2008, 26, 1128-1134.

243. Kasamon, Y.L.; Wahl, R.L. FDG PET and risk-adapted therapy in Hodgkin and non-Hodgkin's lymphoma. Curr. Opin. Oncol. 2008, 20, 206-219.

244. Kasamon, Y.L.; Jones, R.J.; Wahl, R.L. Integrating PET and PET/CT into the risk adapted therapy of lymphoma. J. Nucl. Med. 2007, 48, 19S-27S.

245. Humm, J.L.; Rosenfeld, A.; del Guerra, A. From PET detectors to PET scanners. Eur. J. Nucl. Med. Mol. Imaging 2003, 30, 1574-1597.

246. Tatsumi, M.; Cohade, C.; Nakamoto, Y.; Fishman, E.K.; Wahl, R.L. Direct comparison of FDG PET and CT findings in patients with lymphoma: Initial experience. Radiology 2005, 237, 1038-1045.

247. Boellaard, R.; Oyen, W.J.; Hoekstra, C.J.; Hoekstra, O.S.; Visser, E.P.; Willemsen, A.T.; Arends, B.; Verzijlbergen, F.J.; Zijlstra, J.; Paans, A.M.; et al. The Netherlands protocol for standardisation and quantification of FDG whole body PET studies in multicenter trials. Eur. J. Nucl. Med. Mol. Imaging 2008, 35, 2320-2333.

248. Ziai, D.; Wagner, T.; El Badaoui, A.; Hitzel, A.; Woillard, J.B.; Melloni, B.; Monteil, J. Therapy response evaluation with FDG-PET/CT in small cell lung cancer: A prognostic and comparison study of the PERCIST and EORTC criteria. Cancer Imaging 2013, 13, 73-80.

249. Skougaard, K.; Nielsen, D.; Vittrup Jensen, B.; Westergren Hendel, H. Comparison of EORTC Criteria and PERCIST for PET/CT Response Evaluation of Patients with Metastatic Colorectal Cancer Treated with Irinotecan and Cetuximab. J. Nucl. Med. 2013, 54, 1026-1031.

250. Maffione, A.M.; Ferretti, A.; Chondrogiannis, S.; Rampin, L.; Marzola, M.C.; Grassetto, G.; Capirci, C.; Colletti, P.M.; Rubello, D. Proposal of a new 18F-FDG PET/CT predictor of response in rectal cancer treated by neoadjuvant chemoradiation therapy and comparison with PERCIST criteria. Clin. Nucl. Med. 2013, 38, 795-797.

251. Costelloe, C.M.; Chuang, H.H.; Madewell, J.E.; Ueno, N.T. Cancer Response Criteria and Bone Metastases: RECIST 1.1, MDA and PERCIST. J. Cancer 2010, 1, 80-92. 
252. Cheson, B.D.; Pfistner, B.; Juweid, M.E.; Gascoyne, R.D.; Specht, L.; Horning, S.J.; Coiffier, B.; Fisher, R.I.; Hagenbeek, A.; Zucca, E.; et al. Revised response criteria for malignant lymphoma. J. Clin. Oncol. 2007, 25, 579-586.

253. Juweid, M.E.; Stroobants, S.; Hoekstra, O.S.; Mottaghy, F.M.; Dietlein, M.; Guermazi, A.; Wiseman, G.A.; Kostakoglu, L.; Scheidhauer, K.; Buck, A.; et al. Use of Positron Emission Tomography for Response Assessment of Lymphoma: Consensus of the Imaging Subcommittee of International Harmonization Project in Lymphoma. J. Clin. Oncol. 2007, 25, 571-578.

254. Juweid, M.E.; Cheson, B.D. Role of positron emission tomography in lymphoma. J. Clin. Oncol. 2005, 23, 4577-4580.

255. Fabel, M.; von Tengg-Kobligk, H.; Giesel, F.L.; Bornemann, L.; Dicken, V.; Kopp-Schneider, A.; Moser, C.; Delorme, S.; Kauczor, H.U. Semi-automated volumetric analysis of lymph node metastases in patients with malignant melanoma stage III/IV-A feasibility study. Eur. Radiol. 2008, 18, 1114-1122.

256. Barrington, S.F.; Qian, W.; Somer, E.J.; Franceschetto, A.; Bagni, B.; Brun, E.; Almquist, H.; Loft, A.; Højgaard, L.; Federico, M.; et al. Concordance between four European centres of PET reporting criteria designed for use in multicentre trials in Hodgkin lymphoma. Eur. J. Nucl. Med. Mol. Imaging. 2010, 37, 1824-1833.

257. Cheson, B.D.; Fisher, R.I.; Barrington, S.F.; Cavalli, F.; Schwartz, L.H.; Zucca, E.; Lister, T.A. Recommendations for initial evaluation, staging and response assessment of Hodgkin and nonHodgkin lymphoma - The lugano classification. J. Clin. Oncol. 2014, doi:10.1200/JCO.2013.53.522.

258. Galow, J.R.; Burstein, H.J.; Wood, W.; Hortobagyi, G.N.; Gianni, L.; von Minckwitz, G.; Buzdar, A.U.; Smith, I.E.; Symmans, W.F.; Singh, B.; et al. Preoperative therapy in invasive breast cancer: Pathologic assessment and systemic therapy issues in operable disease. J. Clin. Oncol. 2008, 26, 814-819.

259. Mauri, D.; Pavlidis, N.; Ioannidis, J.P. Neoadjuvant versus adjuvant systemic treatment in breast cancer: A meta-analysis. J. Natl. Cancer Inst. 2005, 97, 188-194.

260. Bear, H.D.; Anderson, S.; Smith, R.E.; Geyer, C.E., Jr.; Mamounas, E.P.; Fisher, B.; Brown, A.M.; Robidoux, A.; Margolese, R.; Kahlenberg, M.S.; et al. Sequential preoperative or postoperative docetaxel added to preoperative doxorubicin plus cyclophosphamide for operable breast cancer: National Surgical Adjuvant Breast and Bowel Project Protocol B-27. J. Clin. Oncol. 2006, 24, 2019-2027.

261. Bonadonna, G.; Valagussa, P.; Brambilla, C.; Ferrari, L.; Moliterni, A.; Terenziani, M.; Zambetti, M. Primary chemotherapy in operable breast cancer: Eight-year experience at the Milan Cancer Institute. J. Clin. Oncol. 1998, 16, 93-100.

262. Fisher, E.R.; Wang, J.; Bryant, J.; Fisher, B.; Mamounas, E.; Wolmark, N. Pathobiology of preoperative chemotherapy: Findings from the National Surgical Adjuvant Breast and Bowel (NSABP) protocol B-18. Cancer 2002, 95, 681-695.

263. Honkoop, A.H.; van Diest, P.J.; de Jong, J.S.; Linn, S.C.; Giaccone, G.; Hoekman, K.; Wagstaff, J.; Pinedo, H.M. Prognostic role of clinical, pathological and biological characteristics in patients with locally advanced breast cancer. Br. J. Cancer 1998, 77, 621-626. 
264. Fisher, B.; Bryant, J.; Wolmark, N.; Mamounas, E.; Brown, A.; Fisher, E.R.; Wickerham, D.L.; Begovic, M.; DeCillis, A.; Robidoux, A.; et al. Effect of preoperative chemotherapy on the outcome of women with operable breast cancer. J. Clin. Oncol. 1998, 16, 2672-2685.

265. Chollet, P.; Amat, S.; Cure, H.; de Latour, M.; Le Bouedec, G.; Mouret-Reynier, M.A.; Ferriere, J.P.; Achard, J.L.; Dauplat, J.; Penault-Llorca, F. Prognostic significance of a complete pathological response after induction chemotherapy in operable breast cancer. Br. J. Cancer 2002, 86, 1041-1046.

266. Gonzalez-Angulo, A.M.; McGuire, S.E.; Buchholz, T.A.; Tucker, S.L.; Kuerer, H.M.; Rouzier, R.; Kau, S.W.; Huang, E.H.; Morandi, P.; Ocana, A.; et al. Factors predictive of distant metastases in patients with breast cancer who have a pathologic complete response after neoadjuvant chemotherapy. J. Clin. Oncol. 2005, 23, 7098-7104.

267. Isasi, C.R.; Moadel, R.M.; Blaufox, M.D. A meta-analysis of FDG-PET for the evaluation of breast cancer recurrence and metastases. Breast Cancer Res. Treat. 2005, 90, 105-112.

268. Du, Y.; Cullum, I.; Illidge, T.M.; Ell, P.J. Fusion of metabolic function and morphology: Sequential $[18 \mathrm{~F}]$ fluorodeoxyglucose positron-emission tomography/computed tomography studies yield new insights into the natural history of bone metastases in breast cancer. J. Clin. Oncol. 2007, 25, 3440-3447.

269. Hinshaw, J.L.; Lee, F.T., Jr. Cryoablation for liver cancer. Tech. Vasc. Interv. Radiol. 2007, 10, 47-57.

270. Wood, T.F.; Rose, D.M.; Chung, M.; Allegra, D.P.; Foshag, L.J.; Bilchik, A.J. Radiofrequency ablation of 231 unresectable hepatic tumors: Indications, limitations, and complications. Ann. Surg. Oncol. 2000, 7, 593-600.

271. Sharma, R.A.; Van Hazel, G.A.; Morgan, B.; Berry, D.P.; Blanshard, K.; Price, D.; Bower, G.; Shannon, J.A.; Gibbs, P.; Steward, W.P. Radioembolization of liver metastases from colorectal cancer using Yttrium-90 microspheres with concomitant systemic oxaliplatin, fluorouracil, and leucovorin chemotherapy. J. Clin. Oncol. 2007, 25, 1099-1106.

272. Bester, L.; Meteling, B.; Pocock, N.; Saxena, A.; Chua, T.C.; Morris, D.L. Radioembolisation with Yttrium-90 microspheres: An effective treatment modality for unresectable liver metastases. J. Med. Imaging Radiat. Oncol. 2013, 57, 72-80.

273. Langenhoff, B.S.; Oyen, W.J.; Jager, G.J.; Strijk, S.P.; Wobbes, T.; Corstens, F.H.; Ruers, T.J. Efficacy of fluorine-18-deoxyglucose positron emission tomography in detecting tumor recurrence after local ablative therapy for liver metastases: A prospective study. J. Clin. Oncol. 2002, 20, 4453-4458.

274. Joosten, J.; Jager, G.; Oyen, W.; Wobbes, T.; Ruers, T. Cryosurgery and radiofrequency ablation for unresectable colorectal liver metastases. Eur. J. Surg. Oncol. 2005, 31, 1152-1159.

275. Donckier, V.; van Laethem, J.L.; Goldman, S.; van Gansbeke, D.; Feron, P.; Ickx, B.; Wikler, D.; Gelin, M. [F-18] fluorodeoxyglucose positron emission tomography as a tool for early recognition of incomplete tumor destruction after radiofrequency ablation for liver metastases. J. Surg. Oncol. 2003, 84, 215-223.

276. Blokhuis, T.J.; van der Schaaf, M.C.; van den Tol, M.P.; Comans, E.F.; Manoliu, R.A.; van der Sijp, J.R. Results of radio frequency ablation of primary and secondary liver tumors: 
Long-term follow-up with computed tomography and positron emission tomography-18Fdeoxyfluoroglucose scanning. Scand. J. Gastroenterol. Suppl. 2004, 241, 93-97.

277. Veit, P.; Antoch, G.; Stergar, H.; Bockisch, A.; Forsting, M.; Kuehl, H. Detection of residual tumor after radiofrequency ablation of liver metastasis with dual modality PET/CT: Initial results. Eur. Radiol. 2006, 16, 80-87.

278. Salem, R.; Lewandowski, R.J.; Gates, V.L.; Nutting, C.W.; Murthy, R.; Rose, S.C.; Soulen, M.C.; Geschwind, J.F.; Kulik, L.; Kim, Y.H.; et al. Research reporting standards for radioembolization of hepatic malignancies. J. Vasc. Interv. Radiol. 2011, 22, 265-278.

279. Shen, S.; DeNardo, G.L.; Yuan, A.; DeNardo, D.A.; DeNardo, S.J. Planar gamma camera imaging and quantitation of yttrium-90 bremsstrahlung. J. Nucl. Med. 1994, 35, 1381-1389.

280. Rault, E.; Clementel, E.; Vandenberghe, S.; D’Asseler, Y.; van Holen, R.; de Beenhouwer, J.; Staelens, S. Comparison of yttrium-90 SPECT and PET images. J. Nucl. Med. 2010, 51, 125.

281. Elschot, M.; Nijsen, J.F.; Dam, A.J.; de Jong, H.W. Quantitative evaluation of scintillation camera imaging characteristics of isotopes used in liver radioembolization. PLoS One 2011, 6, e26174.

282. Selwyn, R.G.; Nickles, R.J.; Thomadsen, B.R.; DeWerd, L.A.; Micka, J.A. A new internal pair production branching ratio of $90 \mathrm{Y}$ : The development of a non-destructive assay for $90 \mathrm{Y}$ and 90Sr. Appl. Radiat. Isot. 2007, 65, 318-327.

283. Lhommel, R.; van Elmbt, L.; Goffette, P.; van den Eynde, M.; Jamar, F.; Pauwels, S.; Walrand, S. Feasibility of $90 \mathrm{Y}$ TOF PET-based dosimetry in liver metastasis therapy using SIR-spheres. Eur. J. Nucl. Med. Mol. Imaging 2010, 37, 1654-1662.

284. Van Elmbt, L.; Vandenberghe, S.; Walrand, S.; Pauwels, S.; Jamar, F. Comparison of yttrium-90 quantitative imaging by TOF and non-TOF PET in a phantom of liver selective internal radiotherapy. Phys. Med. Biol. 2011, 56, 6759-6777.

285. Walrand, S.; Flux, G.D.; Konijnenberg, M.W.; Valkema, R.; Krenning, E.P.; Lhommel, R.; Pauwels, S.; Jamar, F. Dosimetry of yttrium-labelled radiopharmaceuticals for internal therapy: 86 Y or 90 Y imaging? Eur. J. Nucl. Med. Mol. Imaging 2011, 38, S57-S68.

286. Wong, R.K.; Tandan, V.; de Silva, S.; Figueredo, A. Pre-operative radiotherapy and curative surgery for the management of localized rectal carcinoma. Cochrane Database Syst. Rev. 2007, CD002102.

287. Bosset, J.F.; Collette, L.; Calais, G.; Maingon, P.; Radosevic-Jelic, L.; Daban, A.; Bardet, E.; Beny, A.; Ollier, J.C. Chemotherapy with preoperative radiotherapy in rectal cancer. N. Engl. J. Med. 2006, 355, 1114-1123.

288. Hospers, G.A.; Punt, C.J.; Tesselaar, M.E.; Cats, A.; Havenga, K.; Leer, J.W.; Marijnen, C.A.; Jansen, E.P.; van Krieken, H.H.; Wiggers, T.; et al. Preoperative chemoradiotherapy with capecitabine and oxaliplatin in locally advanced rectal cancer: A phase I-II multicenter study of the Dutch Colorectal Cancer Group. Ann. Surg. Oncol. 2007, 14, 2773-2779.

289. De Geus-Oei, L.F.; Vriens, D.; van Laarhoven, H.W.M.; van der Graaf, W.T.A.; Oyen, W.J.G. Monitoring and Predicting Response to Therapy with 18F-FDG PET in Colorectal Cancer: A Systematic Review. J. Nucl. Med. 2009, 50, 43S-54S. 
290. Bipat, S.; Glas, A.S.; Slors, F.J.; Zwinderman, A.H.; Bossuyt, P.M.; Stoker, J. Rectal cancer: Local staging and assessment of lymph node involvement with endoluminal US, CT, and MR imaging - A meta-analysis. Radiology 2004, 232, 773-783.

291. Kwok, H.; Bissett, I.P.; Hill, G.L. Preoperative staging of rectal cancer. Int. J. Colorectal. Dis. 2000, 15, 9-20.

292. Kubota, R.; Yamada, S.; Kubota, K.; Ishiwata, K.; Tamahashi, N.; Ido, T. Intratumoral distribution of fluorine-18-fluorodeoxyglucose in vivo: High accumulation in macrophages and granulation tissues studied by microautoradiography. J. Nucl. Med. 1992, 33, 1972-1980.

293. Larson, S.M. Cancer or inflammation? A Holy Grail for nuclear medicine. J. Nucl. Med. 1994, 35, 1653-1655.

294. Amthauer, H.; Denecke, T.; Rau, B.; Hildebrandt, B.; Hünerbein, M.; Ruf, J.; Schneider, U.; Gutberlet, M.; Schlag, P.M.; Felix, R.; et al. Response prediction by FDG-PET after neoadjuvant radiochemotherapy and combined regional hyperthermia of rectal cancer: Correlation with endorectal ultrasound and histopathology. Eur. J. Nucl. Med. Mol. Imaging 2004, 31, 811-819.

295. Janssen, M.H.M.; Ollers, M.C.; van Stiphout, R.G.P.M.; Riedl, R.G.; van den Bogaard, J.; Buijsen, J.; Lambin, P.; Lammering, G. PET-based treatment response evaluation in rectal cancer: Prediction andvalidation. Int. J. Radiat. Oncol. Biol. Phys. 2012, 82, 871-876.

296. Mandard, A.M.; Dalibard, F.; Mandard, J.C.; Marnay, J.; Henry-Amar, M.; Petiot, J.F.; Roussel, A.; Jacob, J.H.; Segol, P.; Samama, G.; et al. Pathologic assessment of tumor regression after preoperative chemoradiotherapy of esophageal carcinoma. Clinicopathologic correlations. Cancer 1994, 73, 2680-2686.

297. Jemal, A.; Thun, M.J.; Ries, L.A.; Howe, H.L.; Weir, H.K.; Center, M.M.; Ward, E.; Wu, X.C.; Eheman, C.; Anderson, R.; et al. Annual report to the Nation on the status of cancer, 1975-2005, featuring trends in lung cancer, tobacco use, and tobacco control. J. Natl. Cancer Inst. 2008, 100, 1672-1694.

298. Brundage, M.D.; Davies, D.; Mackillop, W.J. Prognostic factors in non-small cell lung cancer: A decade of progress. Chest 2002, 122, 1037-1057.

299. Wright, G.; Manser, R.L.; Byrnes, G.; Hart, D.; Campbell, D.A. Surgery for non-small cell lung cancer: Systematic review and meta-analysis of randomised controlled trials. Thorax 2006, 61, 597-603.

300. MacManus, M.P.; Hicks, R.J.; Matthews, J.P.; McKenzie, A.; Rischin, D.; Salminen, E.K.; Ball, D.L. Positron emission tomography is superior to computed tomography scanning for response-assessment after radical radiotherapy or chemoradiotherapy in patients with non-smallcell lung cancer. J. Clin. Oncol. 2003, 21, 1285-1292.

301. Benz, M.R.; Herrmann, K.; Walter, F.; Garon, E.B.; Reckamp, K.L.; Figlin, R.; Phelps, M.E.; Weber, W.A.; Czernin, J.; Allen-Auerbach, M.S. (18)FFDG PET/CT for monitoring treatment responses to the epidermal growth factor receptor inhibitor erlotinib. J. Nucl. Med. 2011, 52, 1684-1689.

302. Prestwich, R.J.; Subesinghe, M.; Gilbert, A.; Chowdhury, F.U.; Sen, M.; Scarsbrook, A.F. Delayed response assessment with FDGPET-CT following (chemo) radiotherapy for locally advanced head and neck squamous cell carcinoma. Clin. Radiol. 2012, 67, 966-975. 
303. Ghanooni, R.; Delpierre, I.; Magremanne, M.; Vervaet, C.; Dumarey, N.; Remmelink, M.; Lacroix, S.; Trotta, N.; Hassid, S.; Goldman, S. FDG PET/CT and MRI in the follow-up of head and neck squamous cell carcinoma. Contrast. Media Mol. Imaging 2011, 6, 260-266.

304. McGuire, W.P.; Hoskins, W.J.; Brady, M.F.; Kucera, P.R.; Partridge, E.E.; Look, K.Y.; ClarkePearson, D.L.; Davidson, M. Cyclophosphamide and Cisplatin Compared with Paclitaxel and Cisplatin in Patients with Stage III and Stage IV Ovarian Cancer. N. Engl. J. Med. 1996, 334, 1-6.

305. Marcus, C.S.; Maxwell, G.L.; Darcy, K.M.; Hamilton, C.A.; McGuire, W.P. Current Approaches and Challenges in Managing and Monitoring Treatment Response in Ovarian Cancer. J. Cancer 2014, 5, 25-30.

306. Von Georgi, R.; Schubert, K.; Grant, P.; Munstedt, K. Post-therapy surveillance and after-care in ovarian cancer. Eur. J. Obst. Gynecol. Reprod. Biol. 2004, 114, 228-233.

307. Geurts, S.M.; de Vegt, F.; van Altena, A.M.; Tjan-Heijnen, V.C.; Massuger, L.F.; Adang, E.M.; van Dijck, J.A.; Verbeek, A.L. Impact of routine follow-up examinations on life expectancy in ovarian cancer patients: A simulation study. Int. J. Gynecol. Cancer 2012, 22, 1150-1157.

308. Kew, F.; Galaal, K.; Bryant, A.; Naik, R. Evaluation of follow-up strategies for patients with epithelial ovarian cancer following completion of primary treatment. Cochrane Database Syst. Rev. 2011, doi:10.1002/14651858.CD006119.pub2.

309. Miller, R.E.; Rustin, G.J. How to follow-up patients with epithelial ovarian cancer. Curr. Opin. Oncol. 2010, 22, 498-502.

310. Gadducci, A.; Cosio, S. Surveillance of patients after initial treatment of ovarian cancer. Crit. Rev. Oncol. Hematol. 2009, 71, 43-52.

311. Javitt, M.C. ACR Appropriateness Criteria on staging and follow-up of ovarian cancer. J. Am. Coll. Radiol. 2007, 4, 586-589.

312. Thrall, M.M.; DeLoia, J.A.; Gallion, H.; Avril, N. Clinical use of combined positron emission tomography and computed tomography (FDG-PET/CT) in recurrent ovarian cancer. Gynecol. Oncol. 2007, 105, 17-22.

313. Colombo, N.; Peiretti, M.; Parma, G.; Lapresa, M.; Mancari, R.; Carinelli, S.; Sessa, C.; Castiglione, M. Newly diagnosed and relapsed epithelial ovarian carcinoma: ESMO Clinical Practice Guidelines for diagnosis, treatment and follow-up. Ann. Oncol. 2010, 21, v23-v30.

314. Bhosale, P.; Peungjesada, S.; Wei, W.; Levenback, C.F.; Schmeler, K.; Rohren, E.; Macapinlac, H.A.; Iyer, R.B. Clinical utility of positron emission tomography/computed tomography in the evaluation of suspected recurrent ovarian cancer in the setting of normal CA-125 levels. Int. J. Gynecol. Cancer, 2010, 20, 936-944.

315. Bharwani, N.; Reznek, R.H.; Rockall, A.G. Ovarian Cancer Management: The role of imaging and diagnostic challenges. Eur. J. Radiol. 2011, 78, 41-51.

316. Hoekstra, C.J.; Paglianiti, I.; Hoekstra, O.S.; Smit, E.F.; Postmus, P.E.; Teule, G.J.; Lammertsma, A.A. Monitoring response to therapy in cancer using [18f]-2-fluoro-2-deoxy-dglucose and positron emission tomography: An overview of different analytical methods. Eur. J. Nucl. Med. 2000, 27, 731-743.

317. Tomasi, G.; Turkheimer, F.; Aboagye, E. Importance of quantification for the analysis of PET data in oncology: Review of current methods and trends for the future. Mol. Imaging Biol. 2012, 14, 131-146. 
318. Hoekstra, C.J.; Hoekstra, O.S.; Stroobants, S.G.; Vansteenkiste, J.; Nuyts, J.; Smit, E.F.; Boers, M.; Twisk, J.W.; Lammertsma, A.A. Methods to monitor response to chemotherapy in nonsmall cell lung cancer with 18F-FDG PET. J. Nucl. Med. 2002, 43, 1304-1309.

319. Daisne, J.F.; Duprez, T.; Weynand, B.; Lonneux, M.; Hamoir, M.; Reychler, H.; Grégoire, V. Tumor volume in pyafyngolaryngeal squamous cell carcinoma: Comparison at CT, MR imaging and FGD PET and validation with surgical specimen. Radiology 2004, 233, 93-100.

320. Geets, X.; Lee, J.A.; Bol, A.; Lonneux, M.; Gregoire, V. A gradient-based method for segmenting FDG-PET images: Methodology and validation. Eur. J. Nucl. Med. Mol. Imaging 2007, 34, 1427-1438.

321. Werner-Wasik, M.; Nelson, A.D.; Choi, W.; Arai, Y.; Faulhaber, P.F.; Kang, P.; Almeida, F.D.; Xiao, Y.; Ohri, N.; Brockway, K.D.; et al. What is the best way to contour lung tumors on PET scans? Multiobserver validation of a gradient-based method using a NSCLC digital PET phantom. Int. J. Radiat. Oncol. Biol. Phys. 2012, 82, 1164-1171.

322. Riegel, A.C.; Berson, A.M.; Destian, S.; Ng, T.; Tena, L.B.; Mitnick, R.J,; Wong, P.S. Variability of gross tumor volume delineation in head-and-neck cancer using CT and PET/CT fusion. Int. J. Radiat. Oncol. Biol. Phys. 2006, 65, 726-732.

323. Nestle, U.; Schaefer-Schuler, A.; Kremp, S.; Groeschel, A.; Hellwig, D.; Rübe, C.; Kirsch, C.M. Target volume definition for 18F-FDG PET-positive lymph nodes in radiotherapy of patients with non-small cell lung cancer. Eur. J. Nucl. Med. Mol. Imaging 2007, 34, 453-462.

324. Schinagl, D.A.; Vogel, W.V.; Hoffmann, A.L.; van Dalen, J.A.; Oyen, W.J.; Kaanders, J.H. Comparison of five segmentation tools for $18 \mathrm{~F}$-Fluoro-deoxy-glucosepositron emission tomographybased target volume definition in head and neck cancer. Int. J. Radiat. Oncol. Biol. Phys. 2007, 69, 1282-1289.

325. Erdy, Y.E.; Mawlawi, O.; Larson, S.M.; Imbiaco, M.; Yeung, H.; Finn, R.; Humm, J.L. Segmentation of lung lesion volume by adaptive positron emission tomography image thresholding. Cancer 1997, 80, 2505-2509.

326. Nakamoto, Y.; Zasadny, K.R.; Minn, H.; Wahl, R.L. Reproducibility of common semiquantitative parameters for evaluating lung cancer glucose metabolism with positron emission tomography using 2-deoxy-2-[18f]fluoro-d-glucose. Mol. Imaging Biol. 2002, 4, 171-178.

327. Van Dalen, J.A. A novel iterative method for lesion delineation and volumetric quantification with FDG PET. Nucl. Med. Commun. 2007, 28, 485-493.

328. Daisne, J.F.; Sibomana, M.; Bol, A.; Doumont, T.; Lonneux, M.; Grégoire, V. Tri-dimensional automatic segmentation of PET volumes based on measured source-to-background ratios: Influence of reconstruction algorithms. Radiother. Oncol. 2003, 69, 247-250.

329. Schaefer, A.; Kremp, S.; Hellwig, D.; Rübe, C.; Kirsch, C.M.; Nestle, U. A contrast-oriented algorithm for FDG-PET-based delineation of tumour volumes for the radiotherapy of lung cancer: Derivation from phantom measurements and validation in patient data. Eur. J. Nucl. Med. Mol. Imaging 2008, 35, 1989-1999.

330. Zaidi, H.; El Naqa, I. PET-guided delineation of radiation therapy treatment volumes: A survey of image segmentation techniques. Eur. J. Nucl. Med. Mol. Imaging 2010, 37, 2165-2187. 
331. El Naqa, I.; Yang, D.; Apte, A.; Khullar, D.; Mutic, S.; Zheng, J.; Bradley, J.D.; Grigsby, P.; Deasy, J.O. Concurrent multimodality image segmentation by active contours for radiotherapy treatment planning. Med. Phys. 2007, 34, 4738-4749.

332. Li, H.; Thorstad, W.L.; Biehl, K.J.; Laforest, R.; Su, Y.; Shoghi, K.I.; Donnelly, E.D.; Low, D.A.; Lu, W. A novel PET tumor delineation method based on adaptive region-growing and dual-front active contours. Med. Phys. 2008, 35, 3711-3721.

333. Yu, H.; Caldwell, C.; Mah, K.; Mozeg, D. Coregistered FDG PET/CT-based textural characterization of head and neck cancer for radiation treatment planning. IEEE Trans. Med. Imaging 2009, 28, 374-383.

334. Belhassen, S.; Zaidi, H. A novel fuzzy c-means algorithm for unsupervised heterogeneous tumor quantification in PET. Med. Phys. 2010, 37, 1309-1324.

335. Murchison, J.T. TNM staging update for lung cancer: Why is this important? World. J. Radiol. 2012, 4, 126-127.

336. Van de Wiele, C.; Kruse, V.; Smeets, P.; Shathekge, M.; Maes, A. Predictive and prognostic value of metabolic tumor volume and total lesion glycolysis in solid tumors. Eur. J. Nucl. Med. Mol. Imaging 2013, 40, 290-231.

337. Van der Schroeff, M.P.; Baatenburg de Jong, R.J. Staging and prognosis in head and neck cancer. Oral Oncol. 2009, 45, 356-360.

338. Chung, M.K.; Jeong, H.S.; Son, Y.I.; So, Y.K.; Park, G.Y.; Choi, J.Y.; Hyun, S.H.; Kim, H.J.; Ko, Y.H.; Baek, C.H. Metabolic tumor volumes by [18F]-fluorodeoxyglucose PET/CT correlate with occult metastasis in oral squamous cell carcinoma of the tongue. Ann. Surg. Oncol. 2009, 16, 3111-3117.

339. Dibble, E.H.; Alvarez, A.C.; Truong, M.T.; Mercier, G.; Cook, E.F.; Subramaniam, R.M. 18F-FDG metabolic tumor volume and total glycolytic activity of oral cavity and oropharyngeal squamous cell cancer: Adding value to clinical staging. J. Nucl. Med. 2012, 53, 709-715.

340. Chan, S.C.; Chang, J.T.; Lin, C.Y.; Ng, S.H.; Wang, H.M.; Liao, C.T.; Chang, C.J.; Lin, S.Y.; Yen, T.C. Clinical utility of 18F-FDG PET parameters in patients with advanced nasopharyngeal carcinoma: Predictive role for different survival endpoints and impact on prognostic stratification. Nucl. Med. Commun. 2011, 32, 989-996.

341. De Geus-Oei, L.F.; van der Heijden, H.F.; Corstens, F.H.; Oyen, W.J. Predictive and prognostic value of FDG-PET in nonsmall-cell lung cancer: A systematic review. Cancer 2007, 110, $1654-1664$.

342. Lee, P.; Weerasuriya, D.K.; Lavori, P.W.; Quon, A.; Hara, W.; Maxim, P.G.; Le, Q.T.; Wakelee, H.A.; Donington, J.S.; Graves, E.E.; et al. Metabolic tumor burden predicts for disease progression and death in lung cancer. Int. J. Radiat. Oncol. Biol. Phys. 2007, 69, 328-333.

343. Jemal, A.; Siegel, R.; Ward, E.; Hao, Y.; Xu, J.; Thun, M. Cancer statistics. CA Cancer J. Clin. 2009, 59, 225-249.

344. Pennathur, A.; Luketich, J. Resection of esophageal cancer: Strategies for optimal management. Ann. Thorac. Surg. 2008, 85, 751-756.

345. Sagar, P.M.; Gauperaa, T.; Sue-Ling, H.; McMahon, M.J.; Johnston, D. An audit of the treatment of cancer of the oesophagus. Gut 1994, 35, 941-945. 
346. Lordick, F.; Ott, K.; Sendler, A. Gastric cancer and adenocarcinoma of the esophagogastric junction: Principles of neoadjuvant therapy. Chirurg 2011, 82, 968-973.

347. Jayachandran, P.; Pai, R.K.; Quon, A.; Graves, E.; Krakow, T.E.; La, T.; Loo, B.W., Jr.; Koong, A.C.; Chang, D.T. Postchemoradiotherapy positron emission tomography predicts pathologic response and survival in patients with esophageal cancer. Int. J. Radiat. Oncol. Biol. Phys. 2012, 84, 471-477.

348. Zhong, X.; Yu, J.; Zhang, B.; Mu, D.; Zhang, W.; Li, D.; Han, A.; Song, P.; Li, H.; Yang, G.; et al. Using 18F-fluorodeoxyglucose positron emission tomography to estimate the length of gross tumor in patients with squamous cell carcinoma of the esophagus. Int. J. Radiat. Oncol. Biol. Phys. 2009, 73, 136-141.

349. Mamede, M.; El Fakhri, G.; Abreu-e-Lima, P.; Gandler, W.; Nosé, V.; Gerbaudo, V.H. Pre-operative estimation of esophageal tumor metabolic length in FDG-PET images with surgical pathology confirmation. Ann. Nucl. Med. 2007, 21, 553-562.

350. Roedl, J.B.; Harisinghani, M.G.; Colen, R.R.; Fischman, A.J.; Blake, M.A.; Mathisen, D.J.; Mueller, P.R. Assessment of treatment response and recurrence in esophageal carcinoma based on tumor length and standardized uptake value on positron emission tomography-computed tomography. Ann. Thorac. Surg. 2008, 86, 1131-1138.

351. Eifel, P.J.; Winter, K.; Morris, M.; Levenback, C.; Grigsby, P.W.; Cooper, J.; Rotman, M.; Gershenson, D.; Mutch, D.G. Pelvic irradiation with concurrent chemotherapy versus pelvic and para-aortic irradiation for high-risk cervical cancer: An update of radiation therapy oncology group trial (RTOG) 90-01. J. Clin. Oncol. 2004, 22, 872-880.

352. Kidd, E.A.; Thomas, M.; Siegel, B.A.; Dehdashti, F.; Grigsby, P.W. Changes in cervical cancer FDG uptake during chemoradiation and association with response. Int. J. Radiat. Oncol. Biol. Phys. 2013, 85, 116-122.

353. Malpas, J.S.; Caroll, J.J. Myeloma: Clinical presentation and diagnosis. In Myeloma: Biology and Management; Malpas, J.S., Bergsagel, D.E., Kyle, R.A., Eds.; Oxford University Press: New York, NY, USA, 1995; p. 169.

354. Drach, J.; Sagaster, V.; Ackermann, J.; Kaufmann, H. Prognostic factors for multiple myeloma. Hematology 2006, 2, 196-200.

355. Kyle, R.A.; Rajkumar, S.V. Criteria for diagnosis, staging, risk stratification and response assessment of multiple myeloma. Leukemia 2009, 23, 3-9.

356. Durie, B.G.; Salmon, S.E. A clinical staging system for multiple myeloma: Correlation of measured myeloma cells with presenting clinical features, response to treatment and survival. Cancer 1975, 36, 842-854.

357. Durie, B.G.M. The role of anatomical and functional staging myeloma: Description of Durie/Salmon plus staging system. Eur. J. Cancer 2006, 42, 1539-1543.

358. Zamagni, E.; Patriarca, F.; Nanni, C.; Zannetti, B.; Englaro, E.; Pezzi, A.; Tacchetti, P.; Buttignol, S.; Perrone, G.; Brioli, A.; et al. Prognostic relevance of 18-F FDG PET/CT in newly diagnosed multiple myeloma patients treated with up-front autologous transplantation. Blood 2011, 118, 5989-5995. 
359. Fonti, L.; Larobina, M.; del Vecchio, S.; de Luca, S.; Fabbricini, R.; Catalano, L.; Pane, F.; Salvatore, M.; Pace, L. Metabolic Tumor Volume assessed by 18 F-FDG PET/CT for the prediction of outcome in patients with multiple myeloma. J. Nucl. Med. 2012, 53, 1829-1835.

360. Special Report: Positron Emission Tomography for the Indication of Post-Treatment Surveillance of Cancer. Available online: http://www.bcbs.com/blueresources/tec/press/specialreport-positron.html (accessed on 25 July 2014).

361. Podoloff, D.A.; Advani, R.H.; Allred, C.; Benson, A.B., 3rd; Brown, E.; Burstein, H.J.; Carlson, R.W.; Coleman, R.E.; Czuczman, M.S.; Delbeke, D.; et al. NCCN task force report: Positron emission tomography (PET)/computed tomography (CT) scanning in cancer. J. Natl. Compr. Cancer Netw. 2007, 5, S1-S23.

362. Patel, K.; Hadar, N.; Lee, J.; Siegel, B.A.; Hillner, B.E.; Lau, J. The lack of evidence for PET or PET/CT surveillance of patients with treated lymphoma, colorectal cancer, and head and neck cancer: A systematic review. J. Nucl. Med. 2013, 54, 1518-1527.

363. Lee, A.I.; Zuckerman, D.S.; van den Abbeele, A.D.; Aquino, S.L.; Crowley, D.; Toomey, C.; Lacasce, A.S.; Feng, Y.; Neuberg, D.S.; Hochberg, E.P. Surveillance imaging of Hodgkin lymphoma patients in first remission: A clinical and economic analysis. Cancer 2010, 116, 3835-3842.

364. Sobhani, I.; Tiret, E.; Lebtahi, R.; Aparicio, T.; Itti, E.; Montravers, F.; Vaylet, C.; Rougier, P.; André, T.; Gornet, J.M.; et al. Early detection of recurrence by 18FDG-PET in the follow-up of patients with colorectal cancer. Br. J. Cancer 2008, 98, 875-880.

365. Hicks, R.J.; Hofman, M.S. Is there still a role for SPECT-CT in oncology in the PET-CT era? Nat. Rev. Clin. Oncol. 2012, 9, 712-720.

366. Beauregard, J.M.; Williams, S.G.; Degrado, T.R.; Roselt, P.; Hicks, R.J. Pilot comparison of F-fluorocholine and F-fluorodeoxyglucose PET/CT with conventional imaging in prostate cancer. J. Med. Imaging Radiat. Oncol. 2010, 54, 325-332.

367. Evangelista, L.; Zattoni, F.; Guttilla, A.; Saladini, G.; Zattoni, F.; Colletti, P.M.; Rubello, D. Choline PET or PET/CT and biochemical relapse of prostate cancer: A systematic review and meta-analysis. Clin. Nucl. Med. 2013, 38, 305-314.

368. Von Eyben, F.E.; Kairemo, K. Meta-analysis of (11)C-choline and (18)F-choline PET/CT for management of patients with prostate cancer. Nucl. Med. Commun. 2014, 35, 221-230.

369. Treglia, G.; Ceriani, L.; Sadeghi, R.; Giovacchini, G.; Giovanella, L. Relationship between prostate-specific antigen kinetics and detection rate of radiolabelled choline PET/CT in restaging prostate cancer patients: A meta-analysis. Clin. Chem. Lab. Med. 2014, 52, 725-733.

370. Umbehr, M.H.; Muntener, M.; Hany, T.; Sulser, T.; Bachmann, L.M. The role of 11C-choline and 18F-fluorocholine positron emission tomography (PET) and PET/CT in prostate cancer: A systematic review and meta-analysis. Eur. Urol. 2013, 64, 106-117.

371. Evangelista, L.; Guttilla, A.; Zattoni, F.; Muzzio, P.C.; Zattoni, F. Utility of choline positron emission tomography/computed tomography for lymph node involvement identification in intermediate- to high-risk prostate cancer: A systematic literature review and meta-analysis. Eur. Urol. 2013, 63, 1040-1048. 
372. Varrone, A.; Asenbaum, S.; Vander Borght, T.; Booij, J.; Nobili, F.; Nagren, K.; Darcourt, J.; Kapucu, O.L.; Tatsch, K.; Bartenstein, P.; et al. EANM procedure guidelines for PET brain imaging using [18F]FDG, version 2. Eur. J. Nucl. Med. Mol. Imaging 2009, 36, 2103-2110.

373. Spence, A.M.; Muzi, M.; Mankoff, D.A.; O’Sullivan, S.F.; Link, J.M.; Lewellen, T.K.; Lewellen, B.; Pham, P.; Minoshima, S.; Swanson, K.; et al. 18F-FDG PET of gliomas at delayed intervals: Improved distinction between tumor and normal gray matter. J. Nucl. Med. 2004, 45, 1653-1659.

374. Prieto, E.; Marti-Climent, J.M.; Dominguez-Prado, I.; Garrastachu, P.; Diez-Valle, R.; Tejada, S.; Aristu, J.J.; Peñuelas, I.; Arbizu, J. Voxel-based analysis of dual-time-point 18F-FDG PET images for brain tumor identification and delineation. J. Nucl. Med. 2011, 52, 865-872.

375. Mehrkens, J.H.; Popperl, G.; Rachinger, W.; Herms, J.; Seelos, K.; Tatsch, K.; Tonn, J.C.; Kreth, F.W. The positive predictive value of O-(2-[18F]fluoroethyl)-L-tyrosine (FET) PET in the diagnosis of a glioma recurrence after multimodal treatment. J. Neurooncol. 2008, 88, 27-35.

376. Fueger, B.J.; Czernin, J.; Cloughesy, T.; Silverman, D.H.; Geist, C.L.; Walter, M.A.; Schiepers, C.; Nghiemphu, P.; Lai, A.; Phelps, M.E.; et al. Correlation of 6-18F-fluoro-L-dopa PET uptake with proliferation and tumor grade in newly diagnosed and recurrent gliomas. J. Nucl. Med. 2010, 51, 1532-1538.

377. Karunanithi, S.; Sharma, P.; Kumar, A.; Khangembam, B.C.; Bandopadhyaya, G.P.; Kumar, R.; Gupta, D.K.; Malhotra, A.; Bal, C. 18F-FDOPA PET/CT for detection of recurrence in patients with glioma: Prospective comparison with 18F-FDG PET/CT. Eur. J. Nucl. Med. Mol. Imaging 2013, 40, 1025-1035.

378. Lizarraga, K.J.; Allen-Auerbach, M.; Czernin, J.; DeSalles, A.A.; Yong, W.H.; Phelps, M.E.; Chen,W. (18)F-FDOPA PET for differentiating recurrent or progressive brain metastatic tumors from late or delayed radiation injury after radiation treatment. J. Nucl. Med. 2014, 55, 30-36.

379. Kratochwil, C.; Combs, S.E.; Leotta, K.; Afshar-Oromieh, A.; Rieken, S.; Debus, J.; Haberkorn, U.; Giesel, F.L. Intra-individual comparison of (1)(8)F-FET and (1)(8)F-DOPA in PET imaging of recurrent brain tumors. Neuro-Oncology 2014, 16, 434-440.

380. Bacca, A.; Chiacchio, S.; Zampa, V.; Carrara, D.; Duce, V.; Congregati, C.; Simi, P.; Taddei, S.; Materazzi, G.; Volterrani, D.; et al. Role of 18F-DOPA PET/CT in diagnosis and follow-up of adrenal and extra-adrenal paragangliomas. Clin. Nucl. Med. 2014, 39, 14-20.

381. Wong, K.K.; Waterfield, R.T.; Marzola, M.C.; Scarsbrook, A.F.; Chowdhury, F.U.; Gross, M.D.; Rubello, D. Contemporary nuclear medicine imaging of neuroendocrine tumours. Clin. Radiol. 2012, 67, 1035-1050.

382. Tehrani, O.S.; Shields, A.F. PET imaging of proliferation with pyrimidines. J. Nucl. Med. 2013, 54, 903-912.

383. Bading, J.R.; Shields, A.F. Imaging of cell proliferation: Status and prospects. J. Nucl. Med. 2008, 49, 64S-80S.

384. Segall, G.; Delbeke, D.; Stabin, M.G.; Even-Sapir, E.; Fair, J.; Sajdak, R.; Smith, G.T. SNM practice guideline for sodium 18F-fluoride PET/CT bone scans 1.0. J. Nucl. Med. 2010, 51, 1813-1820.

385. Hofman, M.S.; Kong, G.; Neels, O.C.; Eu, P.; Hong, E.; Hicks, R.J. High management impact of Ga-68 DOTATATE (GaTate) PET/CT for imaging neuroendocrine and other somatostatin expressing tumours. J. Med. Imaging radiat. Oncol. 2012, 56, 40-47. 
386. Hofmann, M.; Maecke, H.; Borner, R.; Weckesser, E.; Schoffski, P.; Oei, L.; Schumacher, J.; Henze, M.; Heppeler, A.; Meyer, J.; et al. Biokinetics and imaging with the somatostatin receptor PET radioligand (68)Ga-DOTATOC: Preliminary data. Eur. J. Nucl. Med. 2001, 28, 1751-1757.

387. Ambrosini, V.; Campana, D.; Tomassetti, P.; Fanti, S. (6)(8)Ga-labelled peptides for diagnosis of gastroenteropancreatic NET. Eur. J. Nucl. Med. Mol. Imaging 2012, 39, S52-S60.

388. Drzezga, A.; Souvatzoglou, M.; Eiber, M.; Beer, A.J.; Furst, S.; Martinez-Moller, A.; Nekolla, S.G.; Ziegler, S.; Ganter, C.; Rummeny, E.J.; et al. First clinical experience with integrated wholebody PET/MR: Comparison to PET/CT in patients with oncologic diagnoses. J. Nucl. Med. 2012, $53,845-855$.

389. Tian, J.; Fu, L.; Yin, D.; Zhang, J.; Chen, Y.; An, N.; Xu, B. Does the Novel Integrated PET/MRI Offer the Same Diagnostic Performance as PET/CT for Oncological Indications? PLoS One, 2014, 9, e90844.

390. Al-Nabhani, K.Z.; Syed, R.; Michopoulou, S.; Alkalbani, J.; Afaq, A.; Panagiotidis, E.; O’Meara, C.; Groves, A.; Ell, P.; Bomanji, J. Qualitative and quantitative comparison of PET/CT and PET/MR imaging in clinical practice. J. Nucl. Med. 2014, 55, 88-94.

391. Wetter, A.; Lipponer, C.; Nensa, F.; Heusch, P.; Rubben, H.; Schlosser, T.W.; Pöppel, T.D.; Lauenstein, T.C.; Nagarajah, J. Quantitative evaluation of bone metastases from prostate cancer with simultaneous [F] choline PET/MRI: Combined SUV and ADC analysis. Ann. Nucl. Med. 2014, 28, 405-410.

392. Queiroz, M.A.; Hullner, M.; Kuhn, F.; Huber, G.; Meerwein, C.; Kollias, S.; von Schulthess, G.; Veit-Haibach, P. PET/MRI and PET/CT in follow-up of head and neck cancer patients. Eur. J. Nucl. Med. Mol. Imaging 2014, 41, 1066-1045.

393. Littooij, A.S.; Kwee, T.C.; Barber, I.; Granata, C.; Vermoolen, M.A.; Enriquez, G.; Zsíros, J.; Soh, S.Y.; de Keizer, B.; Beek, F.J.; et al. Whole-body MRI for initial staging of paediatric lymphoma: Prospective comparison to an FDG-PET/CT-based reference standard. Eur. Radiol. 2014, 24, 1153-1165.

394. Yoon, S.H.; Goo, J.M.; Lee, S.M.; Park, C.M.; Seo, H.J.; Cheon, G.J. Positron emission tomography/magnetic resonance imaging evaluation of lung cancer: Current status and future prospects. J. Thorac. Imaging 2014, 29, 4-16.

(C) 2014 by the authors; licensee MDPI, Basel, Switzerland. This article is an open access article distributed under the terms and conditions of the Creative Commons Attribution license (http://creativecommons.org/licenses/by/4.0/). 